Historic, Archive Document

Do not assume content reflects current scientific knowledge, policies, or practices. 



\section{Thomas Meehan \& Sons WHOLESALE NURSERYMEN
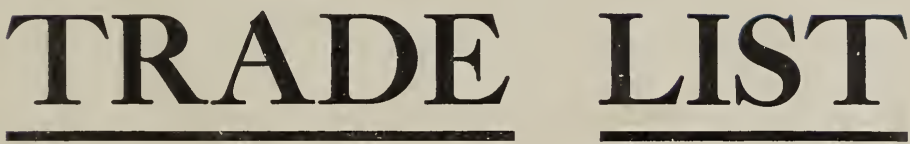 \\ Fall, 1910}

PRICES NET

NO DISCOUNTS
THIS CATALOGUE CANCELS ALL PREVIOUS PRICE LISTS

\section{WHOLESALE DEPARTMENT}

\section{Dresher P. 0., Montgomery County, Penna.}

Postal Telegraph, Dresher, Pa.

Western Union, Ambler, Pa.

Bell Telephone, Ambler 226

\section{ACRES}




\section{TRADE LIST}

\section{Fall, 1910}

CUSTOMERS are requested to state the mode of conveyance by which they desire their trees forwarded. When no route is named, we forw ard to the best of our judgment, without liability for error or delay.

RESPONSIBILITY.-After goods are placed on board cars here, we assume no responsibility for their safe or prompt delivery, or any injury caused by delay in delivery by the transportation company.

ALL CLAIMS must be made upon receipt of goods.

TERMS CASH, on or before delivery to express or railroad agents, unless by special arrangement.

Two hundred and fifty, twenty-five or five of any one variety will be furnished at the thousand, hundred and ten rates, respectively, but single plants of one or more varieties will not be sold at less than 15 cents each except Vines and Herbaceous Plants, the minimum price of which will be ro cents each.

PACKING CHARGED EXTRA, but only sufficient to cover cost of time and material consumed in the work.

POST OFFICE ORDERS payable at Dresher P. O., Montgomery Co., Pa.

POSTAL TELEGRAPH OFFICE, DRESHER, PA. WESTERN UNION, AMBLER, PA. Use Nurserymen's Telegraphic Code or “A. B. C." Code, 4th Edition.

VISITORS are always welcome and should take train at the Reading Terminal, I 2th and Market Streets, Philadelphia, for Camp Hill Station, or at Broad Street Station, Pennsylvania R. R., for Fort Hill Station. Be sure that the train you take stops at these stations.

NOTICE.-It is necessary that you should TELEPHONE us (Bell Phone, 226 Ambler; Keystone Phone, Jenkintown 652-Y), in advance of your coming, so that we can send a carriage to the station for you. Our office is $2 \frac{1}{2}$ miles from either station.

\section{GERTIFICATE OF INSPECTION}

Our nurseries are inspected several times each year, by inspectors of the Entomological Division of the Department of Agriculture of the State of Pennsylvania, and are free from insect pests and diseases. A certificate of the Department is attached to all shipments, and will also be furnished to customers if desired. 


\section{Deciduous Trees}

Several years ago we realized that there would shortly be a demand for a better grade of shade trees than were being commonly sold in nurseries at that time. With this idea in mind we commenced transplanting large blocks of all varieties, selecting only nice, straight, first-class trees for the purpose, discarding any crooked or ill-shaped stock. Time has proven the wisdom of our foresight, and while the demand for this class of stock has been greater than our expectation, our supply has been sufficient to meet the demand, and though our sales last year on these trees were very heavy we still have a good supply on hand for this season's sales.

Our object is to grow a first-class tree, well rooted, with straight trunk and a nice top and we spare no expense to produce a tree of this character. All crooked trees are discarded. Every tree we send out is a good tree. We stake all of our trees with long bamboo poles and in that way we get not only a perfectly straight trunk, but a stem right to the top of the tree. This should be kept in mind when comparing our prices with the "cheap" trees usually offered.

Notice.-When ordering trees, be sure to specify both the height and calliper, as shown in the catalogue. It will be noticed that in many instances trees of the same height are of different calliper and in others they are the same calliper but different height.

\section{Acer campestre, English Cork Maple}

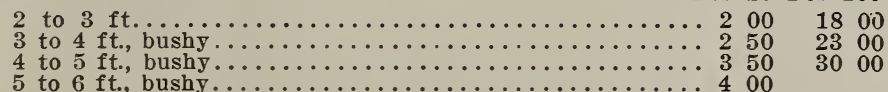

" dasycarpum, Silver Maple

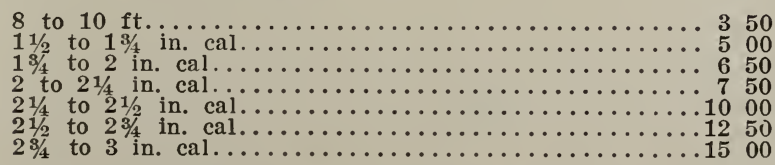

“ dasycarpum Weirii, Weir's Cut-leaf Maple

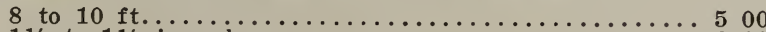

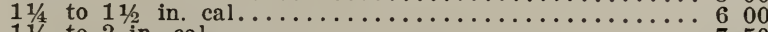

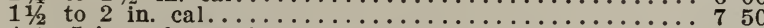

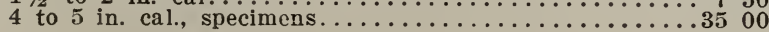


Per 10 Per 100 Per 1000

" negundo, Ash-leaved Maple, Box Elder

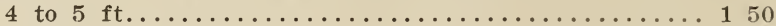

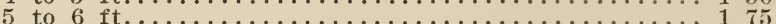

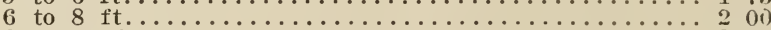

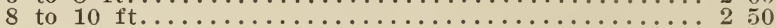

1500

1600

1800

2300

"Pennsylvanicum, see striatum

" platanoides, Norway Maple

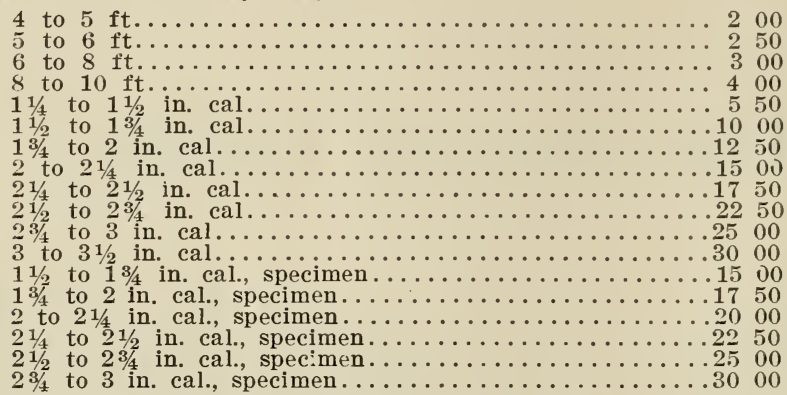

1800

2000

2700

4000

5500

10000

12500

15000

17500

22500

15000

17500

20000

We take a great deal of pride in our Norway

Map!es. We believe there are no finer trees in the country. All trees quoted have been once or more transplated within the last three years. All crooked trees were discarded when the stock was transplated, and every tree we send out has a good straight trunk with good heads and an abundance of fibrous roots. The "specimen" trees have been growing five by five feet apart in the nursery rows, giving ample opportunity to develop good well shaped heads.

" platanoides Reitenbachi, Purple-leaved Norway Maple

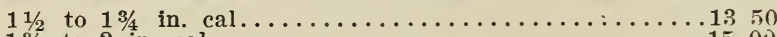

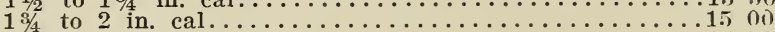

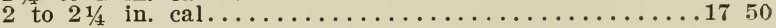

The Reitenbach Maple is a purple-leaved variety. The color is not as strong when the leaves first come out in the spring, but it develops later and retains the color better than the Schwedlerii during the summer months.

“ platanoides Schwedlerii, Purple-leaved Norway Maple

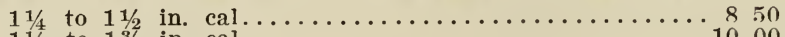

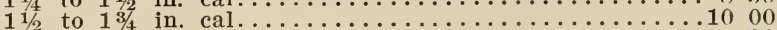

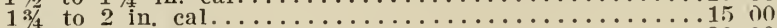

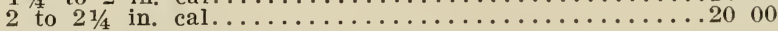

8500 10000 15000

Both the Reitenbach and Schwedler Maples were transplanted three years ago. They are selected trees with perfectly straight trunks and good tops

" platanoides globosa, globe-headed Norway Maple

4 to $5 \mathrm{ft}$., 2 to $3 \mathrm{ft}$., stems............... 750

" pseudo-platanus, Sycamore Maple

$11 / 2$ to $13 \frac{4}{4}$ in. cal....................... 750

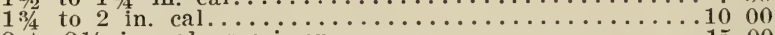

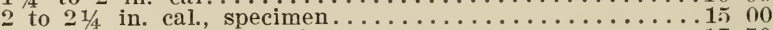

$21 / 4$ to $21 / 2$ in. cal., specimen............... 50

$21 / 2$ to $2 \frac{1}{4}$ in. cal., specimen...................... 00

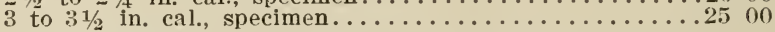

Selected trees, straight trunks, transplanted three years. 


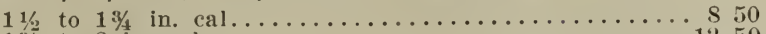

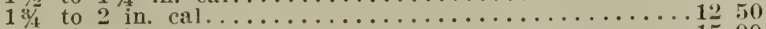

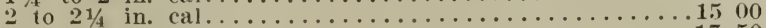

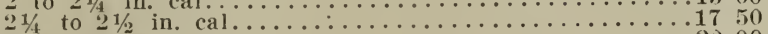

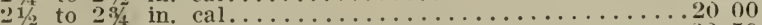

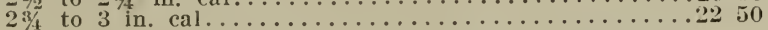

These trees usually make a very crooked trunk with a low, unformed head. Our trees have perfectly straight trunks 6 to 7 feet with fine well shaped heads.

“ rubrum, Red or Scarlet Maple

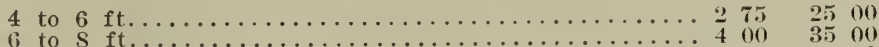

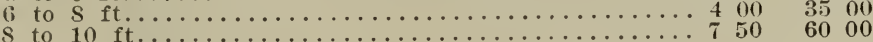

" saccharum, Sugar or Rock Maple

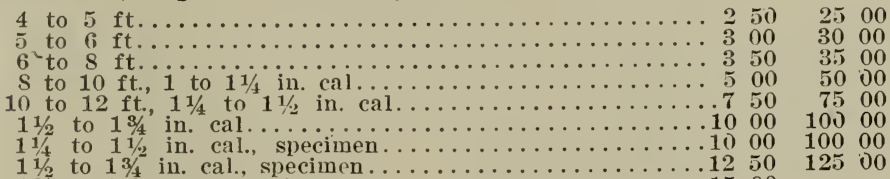

" saccharum nigrum, Black Sugar Maple

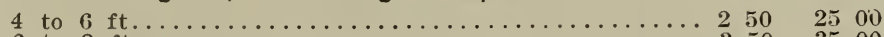

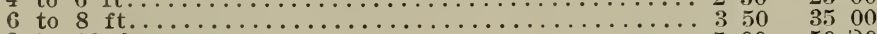

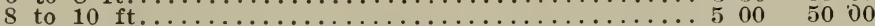

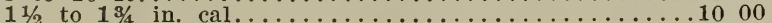

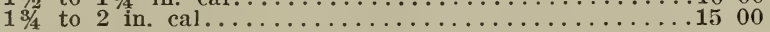

" striatum, (Pennsylvanicum)

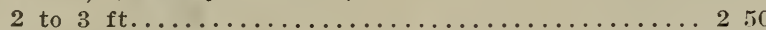

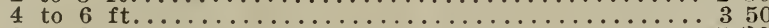

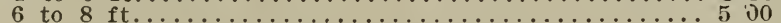

“Tataricum

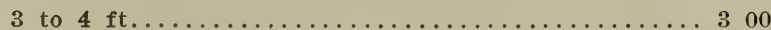

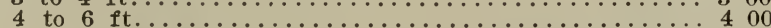

"Tataricum Ginnale

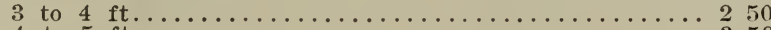

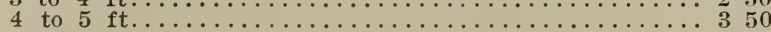

\section{Japanese Maples}

We have a large stock of Japanese Maples, all of our own propagation. Plants imported from Japan are crooked, illshaped and poorly rooted. Our plants have straight stems and good bushy, healthy tops. You will find they are well worth the difference in price. We grow some of nearly all of the varieties in pots, as they transplant much more readily in this way than from the open ground. The ball is preserved by knocking them from the clay pots and sewing the ball in burlap.

Acer Japonicum, Green-leaved

2 to $3 \mathrm{ft}$, field grown.................... 00

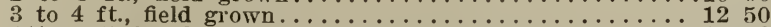

$41 / 2$ to $5 \mathrm{ft}$., field grown, specimen............... 00

3 to $5 \mathrm{ft}, 2$ to $3 \mathrm{ft}$. stems, standards, field grown.....25 00

4 to $6 \mathrm{ft}$., 3 year heads, standards, field grown........25 00

"Japonicum aconitifolium, Green-leaved

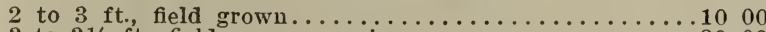

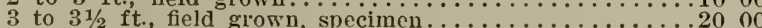

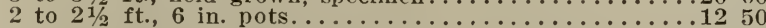

"Japonicum aureum, Golden-leaved

2 to $2 \frac{1}{2} \mathrm{ft}$., field grown. 
"Japonicum palmatum, Green-leaved

Per 10 Per 100 Per 1000

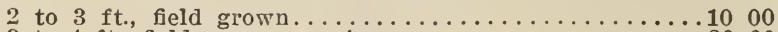

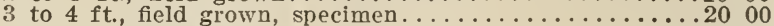

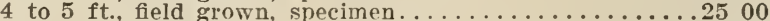

2 to $3 \mathrm{ft}$, 6 in. pots........................ 50

" polymorphum, Green-leaved

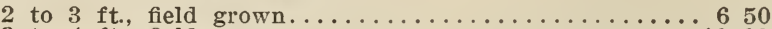

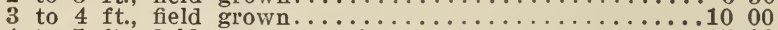

4 to $5 \mathrm{ft}$, field grown, specimen............. 00

5 to $6 \mathrm{ft}$, field grown, heavy specimen...........20 00

4 to $6 \mathrm{ft}$., standards, field grown............. 17

" polymorphum ampelopsilobum, Green-leaved

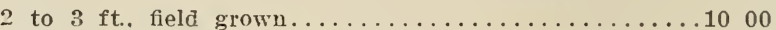

$31 /$ to $4 \mathrm{ft}$, field grown, specimen............. 00

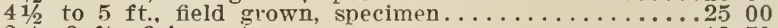

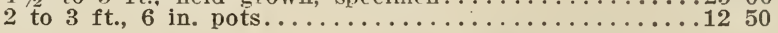

" polymorphum atropurpureum, Blood-leaved

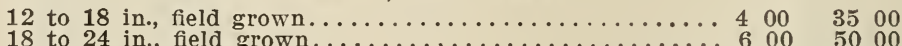

18 to 24 in., field grown...............

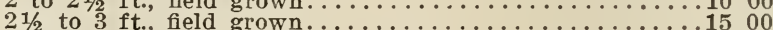

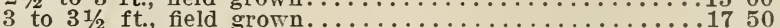

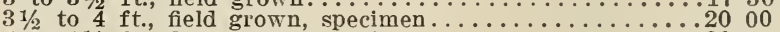

4 to $41 / 2 \mathrm{ft}$., field grown, specimen........................ 00

$41 / 2$ to $5 \mathrm{ft}$, field grown, specimen............40 00

"polymorphum cristatum, Green Fern-leaved

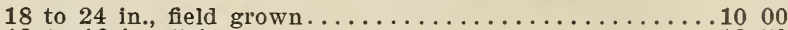

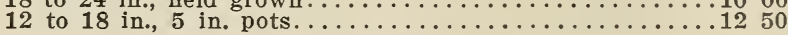

" polymorphum dissectum, Green Cut-leaved

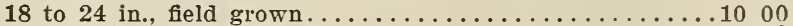

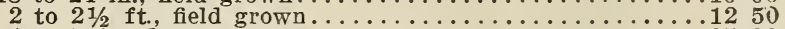

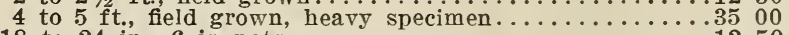

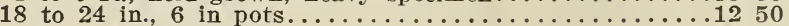

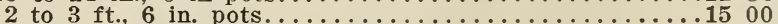

" polymorphum dissectum atropurpureum, Purple Cut-leaved

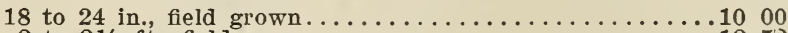

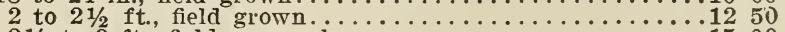

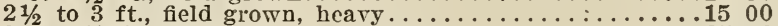

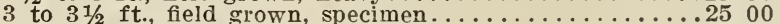

$31 / 2$ to 4 ft., field grown, specimen............................. 00

4 to $5 \mathrm{ft}$., standards, field grown ..................

" polymorphum pinnatifolium atropurpureum, Purple lance-leaved

18 to 24 in., field grown..................... 1000

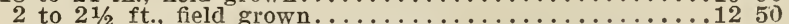

$21 / 2$ to 3 ft., field grown, specimen.....................

" polymorphum reticulatum, Green and yellow variegated leaved

18 to 24 in., field grown.................. 00

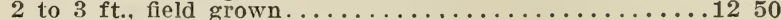

3 to $31 / \mathrm{ft}$., field grown, specimen................ 00

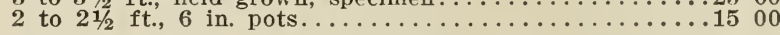

"polymorphum sanguineum, Purple-leaved

18 to 24 in., field grown.................. 60

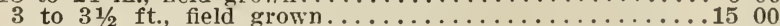

18 to 24 in., 5 in. pots........................ 10

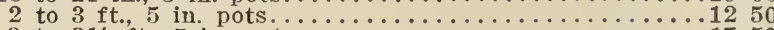

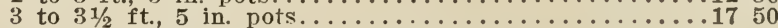

Aesculus hippocastaneum, Eu. Horse Chestnut

$13 / 4$ to 2 in. cal................... 50

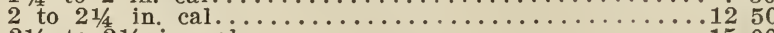

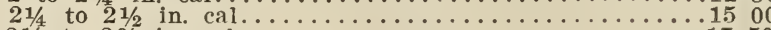

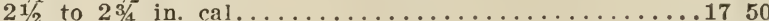

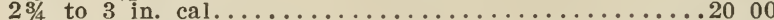

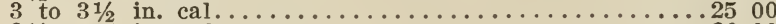

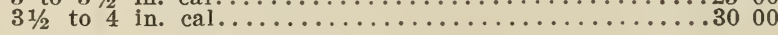

These are fine straight trees. Transplanted three years ago. 
“ hippocastaneum alba plena, Double White Flowered.

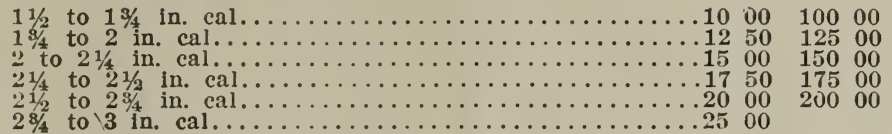

A fine lot of trees. Probably the largest supply in this country. Transplanted three years.

Ailanthus glandulosa, Tree of Heaven

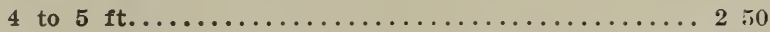

Amygdalus Persica, Double Flowering Peach.-See Shrubs

Aralia.-See Shrubs

Betula alba, Eu. White Birch

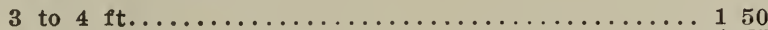

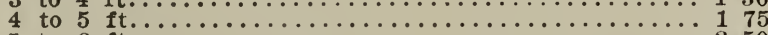

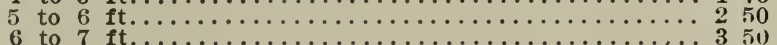

1300

1500

2000

" alba laciniata, Cut-leaf Weeping Birch

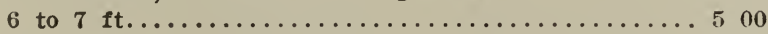

" lenta, Sweet Birch

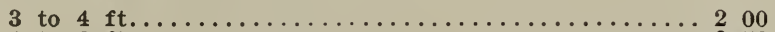

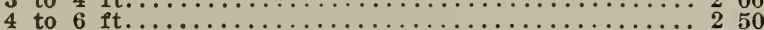

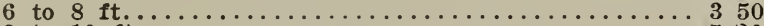

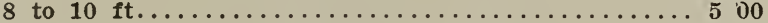

“ lutea, Yellow Birch

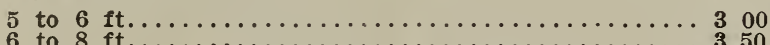

" papyracea, Paper Birch

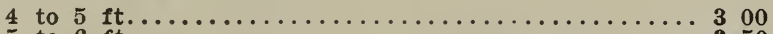

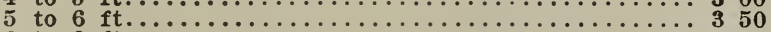

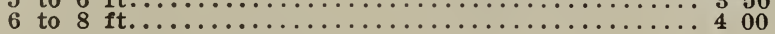

3500 4000

" populifolia, Poplar Birch

3 to 4 ft......................... $00 \quad 2000$

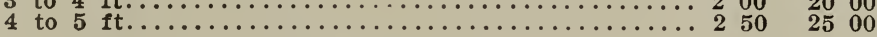

" rubra (nigra), Red or Water Birch

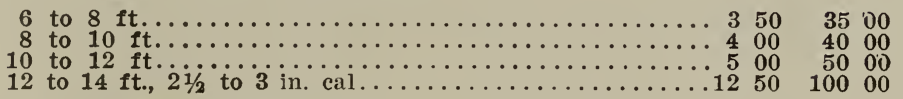

Carpinus. Hornbeam.-See Shrubs

Carya amara

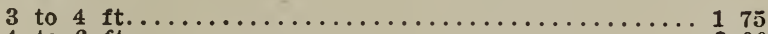

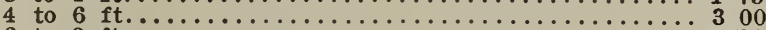

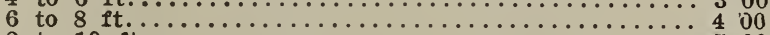

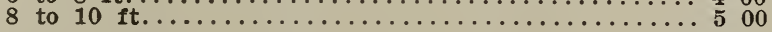

" alba, Shellbark

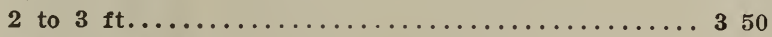

" olivaeformis, Pecan

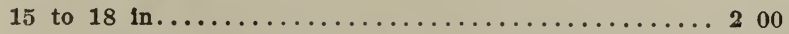

" sulcata, Western Shellbark

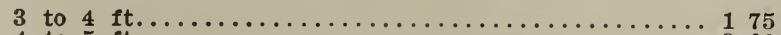

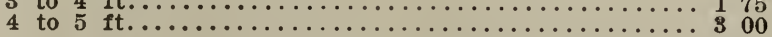

Castanea Americana, Sweet Chestnut

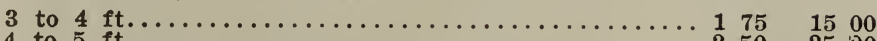

" vesca, Spanish Chestnut

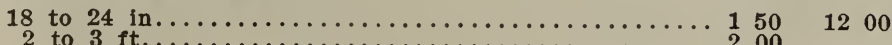


Catalpa Bungeii, Standard

2 year heads 500

"Kaempferi, Japanese

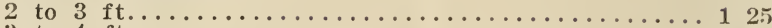

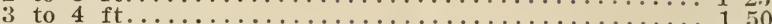

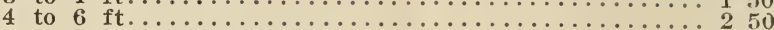

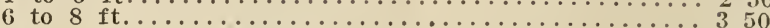

1500

2000

\section{speciosa, Western Catalpa}

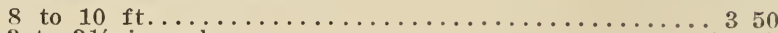

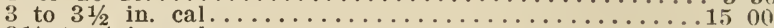

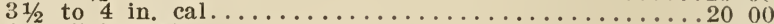

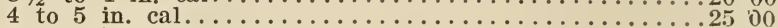

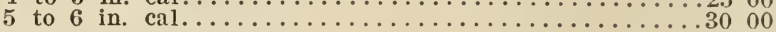

\section{Cedrela sinensis}

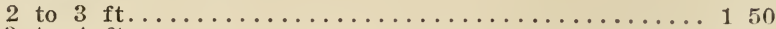

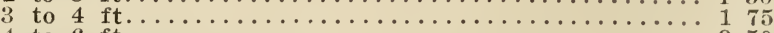

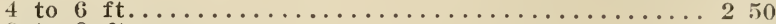

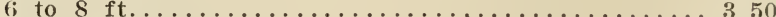

8 to $10 \mathrm{ft} . \ldots . \cdots$

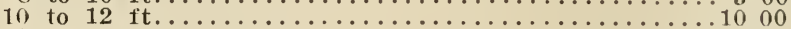

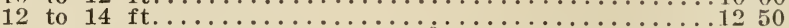

The value of the Cedrela as a shade tree, particularly for planting alongstreets, is not well resognized, though it is one of the best shade trees for this purpose. It is perfectly hardy in Philadelphia and the lower New England States. We have never known it to be attacked by insects or caterpillars. In cities where the streets are narrow and under the most adverse conditions, it thrives equally as well as the Ailanthus and its flowers are entirely free from that disagreeable odor.

Celtis occidentalis, Nettle or Hack-berry

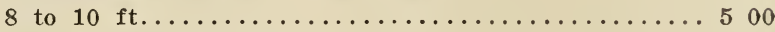

" sinensis, Chinese Hack-berry

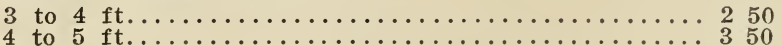

Cerasus Japonica rosea pendula, Weeping Rose FI. Cherry

2 year heads....................... 50

"Padus, European Bird-Cherry

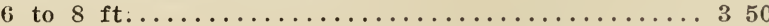

" serotina, American Bird-Cherry

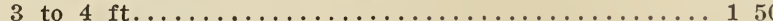

Cercidiphyllum Japonicum

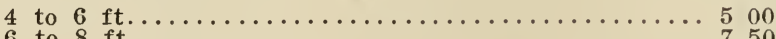

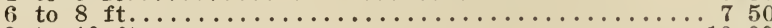

5000 7500

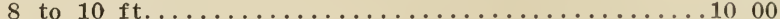

Cercis Canadensis and Japonica, see shrubs

Cladrastis tinctoria (Virgilia lutea), Yellow Wood

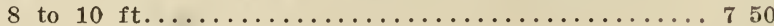

\section{Cornus florida, White Flowered Dogwood}

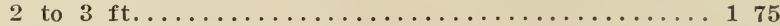

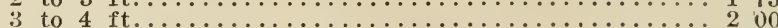

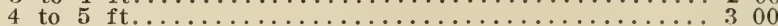

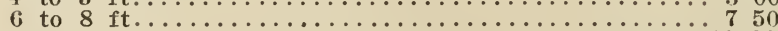

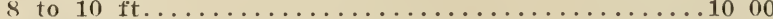

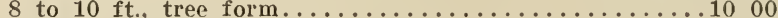

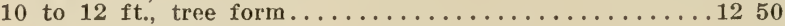


I'er 10 I'er 106 Per 1000

“ florida pendula, Weeping Dogwood

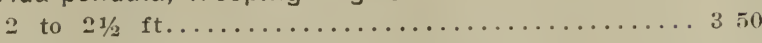

“ florida rubra, Red Flowered Dogwood

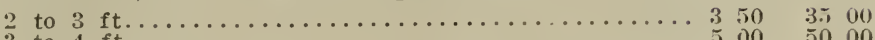

" Other varieties (See Shrubs)

Crataegus (See Shrubs)

Dimorpanthus Mandschurica (See Aralia Mandschurica)

Diospyrus Virginiana, American Persimmon

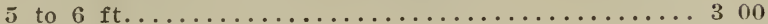

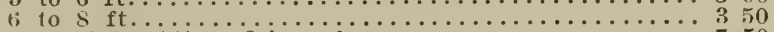

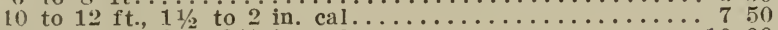

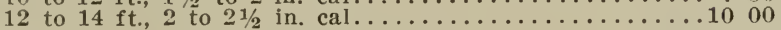

Fagus sylvatica, European Beech

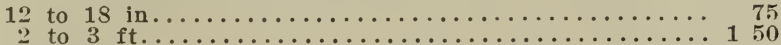

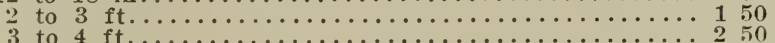

These European Beech will make very satis-

factory hedge plants.

“ asplenifolia, Fern-leaved Beech

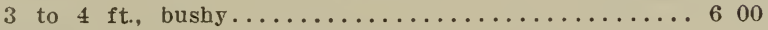

" heterophylla, Cut-leaved Beech

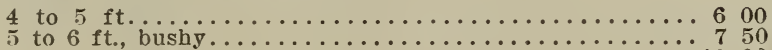

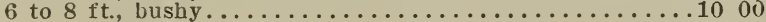

“ purpurea, Copper Beech

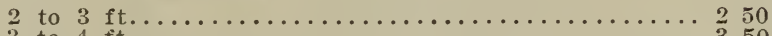

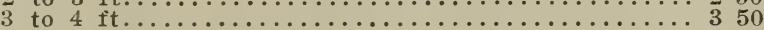

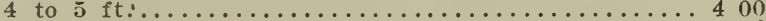

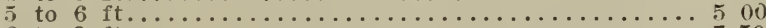

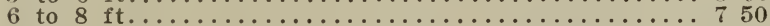

“Rivers' Blood-leaf Beech

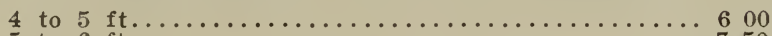

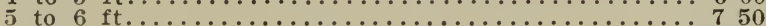

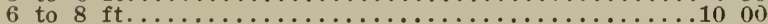

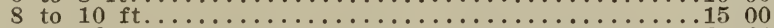

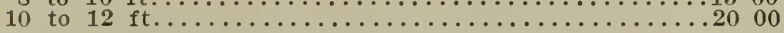

500

1200

2000

All transplanted within the last three years.

They were severely cut back at the time and have made a nice growth, but the branches do not spread as much as might be expected for trees of this height but they are in fine shape for moving. All branched to the ground.

" purpurea pendula, Weeping Blood-leaf Beech

5 to $6 \mathrm{ft}$. .1500

Fraxinus Americana, American White Ash

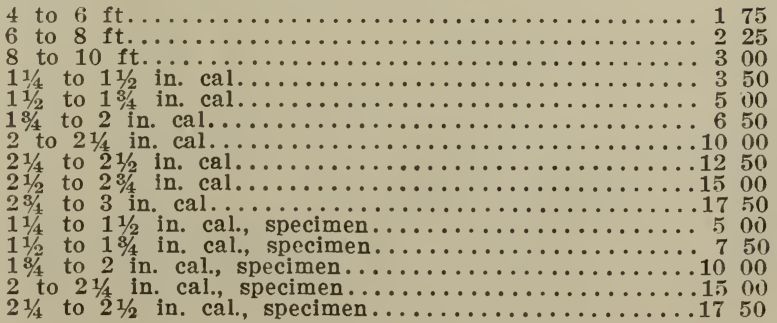


" excelsior, English Ash

Per 10 Per 100 Per 1000

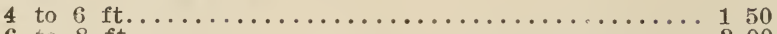

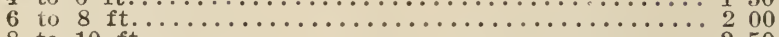

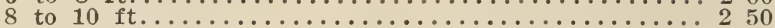

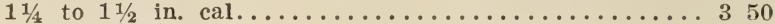

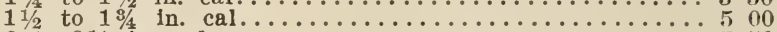

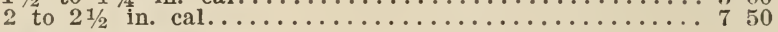

" excelsior acubaefolia

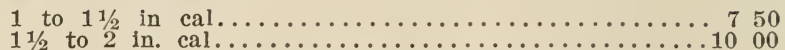

" excelsior aurea pendula, Weeping Golden bark Ash

4 to $5 \mathrm{ft}$, 3 year heads.................. 7 50

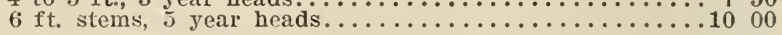

" excelsior heterophylla

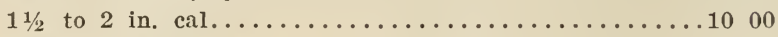

"Ornus, Flowering Ash

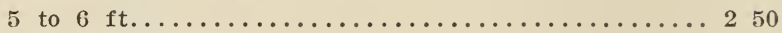

"pubescens, Red Ash

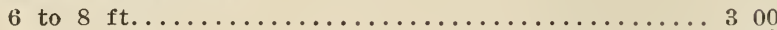

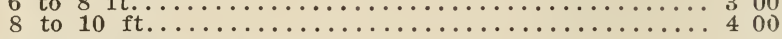

"quadrangulata, Blue Ash

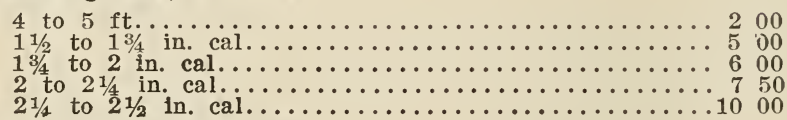

“ sambucifolia, Black Ash

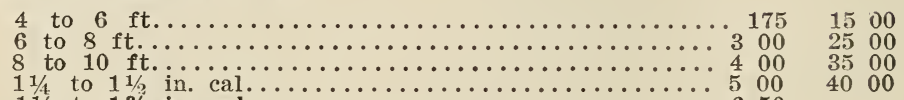

" viridis, Green Ash

$11 / 2$ to $13 / 4$ in. cal.......................... 50

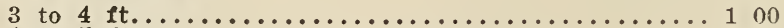

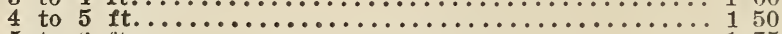

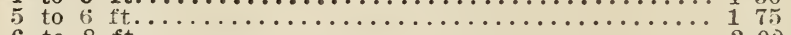

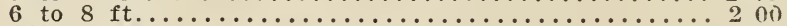

$11 / 2$ to $13 / 4$ in. cal., specimen................ 750

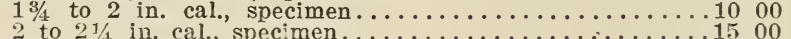

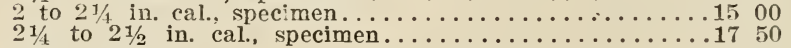

Ginkgo-see Salisburia

Gleditschia triacanthos, Honey Locust

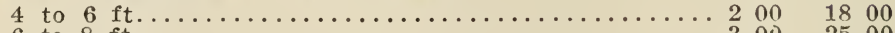

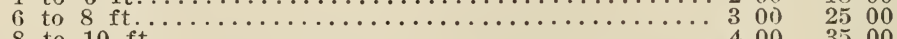

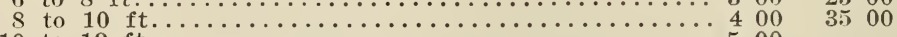

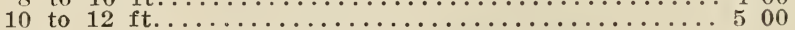

Gymnocladus canadensis, Kentucky Coffee Tree

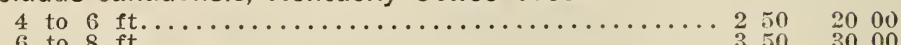

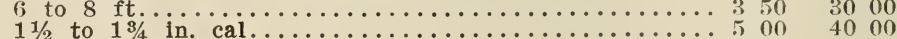

$13 / 4$ to 2 in. cal...............

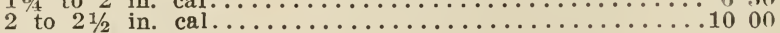

Juglans nigra, Black Walnut

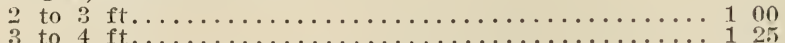

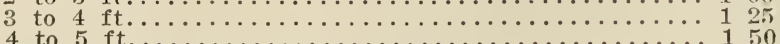

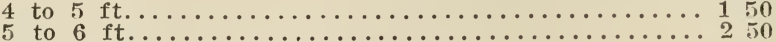

" regia, English Walnut, Madera Nut

18 to 24 in ............................... 75 6 00

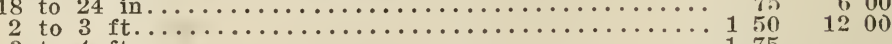

5000

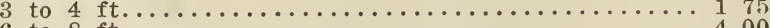

Koelreuteria paniculata. dapanese Varnish Tree

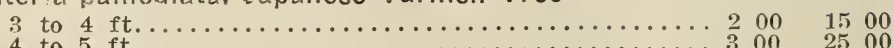

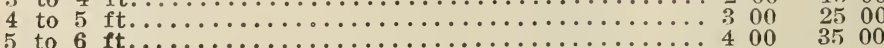


P'er 10 I'er 100 I'er 1000

Liquidambar styraciflua, Sweet Gum

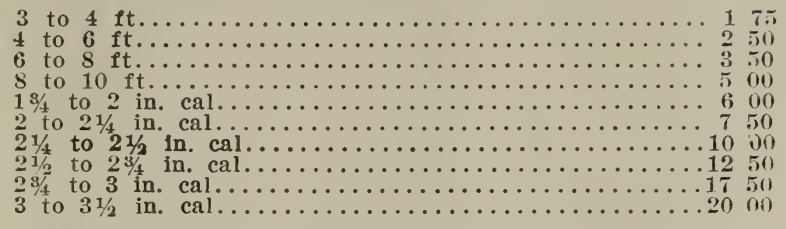

1500 2000 4000

We grow only the "hardy" Sweet Gum. We collect our seeds from trees growing in this vicinity and from them produce a strain hardy throughout the New England States. Seedlings from southern trees are not hardy as far north as Philadelphia.

Liriodendron tulipifera, Tulip Tree

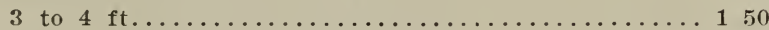

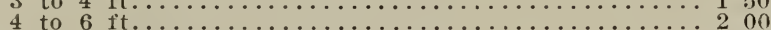

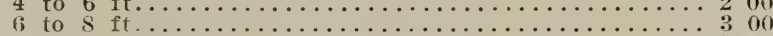

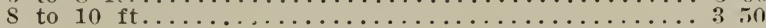

Magnolia acuminata

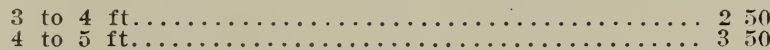

"glauca

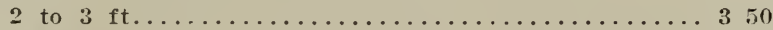

" tripetela

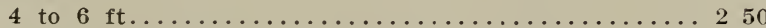

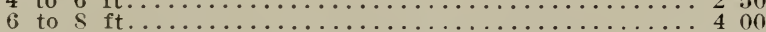

\section{Magnolias}

\section{Large Flowered Chinese Varieties}

The following Magnolias have been several times transplanted. They are very bushy plants, branched to the ground. They will be dug with ball of earth and the ball sewed in burlap.

"Alexandrina, White, pink centre

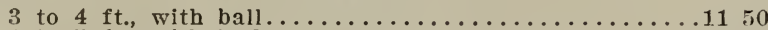

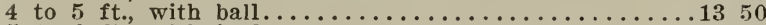

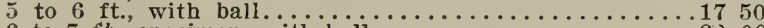

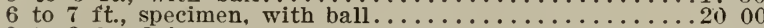

2 to $3 \mathrm{ft}$., 8 in. pots...................... 50

“ conspicua, Chinese White Magnolia

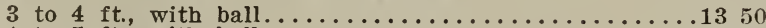

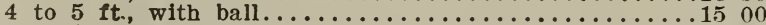

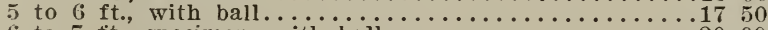

6 to $\tau$ ft.. specimen, with bali......................

\section{"Halleana,-see stellata}

\section{"Kobus, White}

18 to 24 in., with ball.................. 500

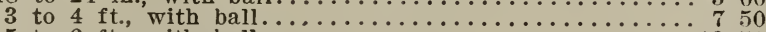

5 to $6 \mathrm{ft}$, with ball......................

"Lennei, Large Purple Flowered

3 to $4 \mathrm{ft}$, with ball.................. 1350

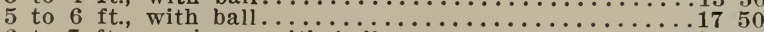

6 to 7 ft., specimen. with bali......................

" purpurea, Purple

18 to 24 in., with ball.................. 500

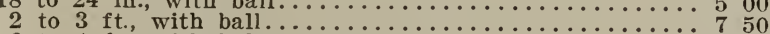

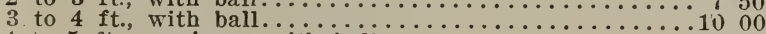

4 to $5 \mathrm{ft}$., specimen, with bali.................. 00 
"Soulangeana, Large Pink Flowered Magnolia

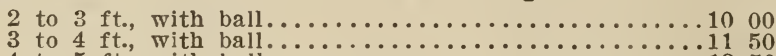

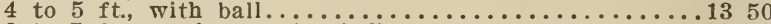

6 to $7 \mathrm{ft.}$, specimen, with ball................... 00

"Soulangeana nigra,

2 to $2 \frac{1}{2} \mathrm{ft}$, with ball...................... 50

" speciosa, Early Pink Flowered Magnolia

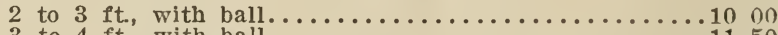

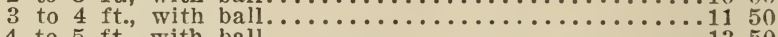

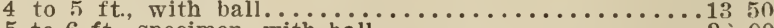

5 to $6 \mathrm{ft}$, specimen, with ball.................

“ stellata (Halleana), Hall's White Star Flowered

2 to $21 / \frac{1}{5} \mathrm{ft}$, with ball........................... 50

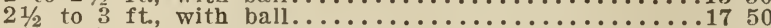

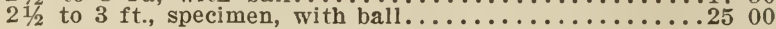

Morus Tartarica, Russian Mulberry

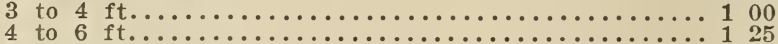

800

“Tea's Weeping Mulberry

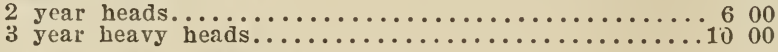

Negundo aceroides. See Acer negundo

Nyssa multiflora

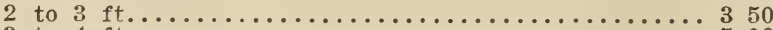

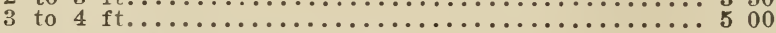

Oxydendron arboreum, Sorrel Tree

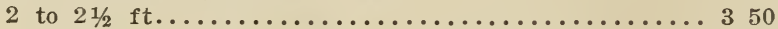

Paulownia imperialis, Empress Tree

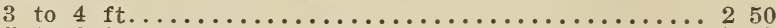

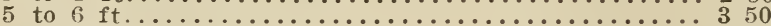

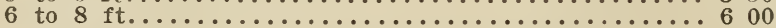

Pavia rubra

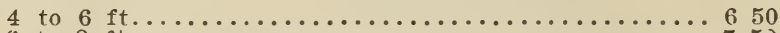

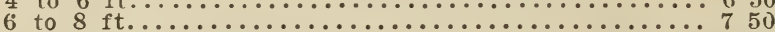

" flava, Yellow Buckeye

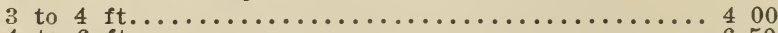

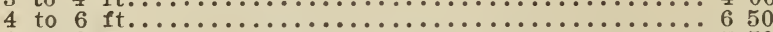

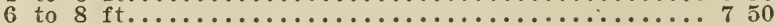

Phellodendron amurense

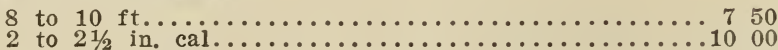

Platanus orientalis, European Sycamore, European Plane

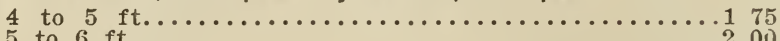

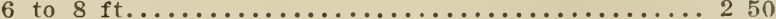

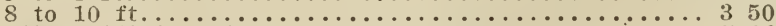

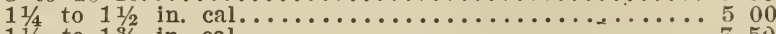

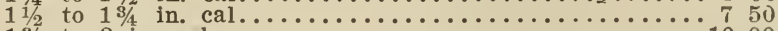

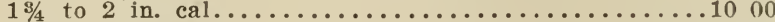

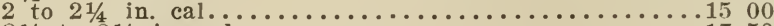

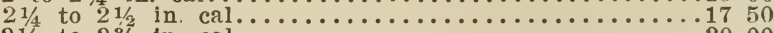

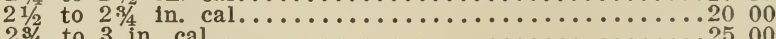

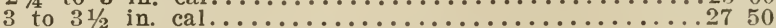

2000

1000

\section{(10)}

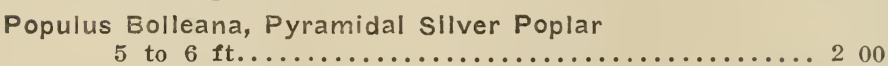

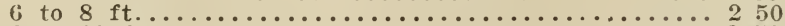

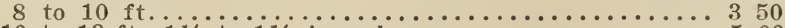

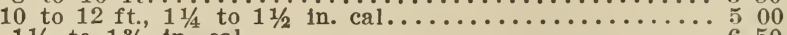

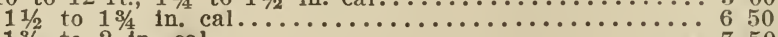

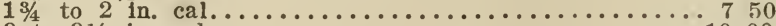

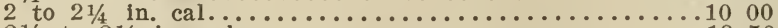

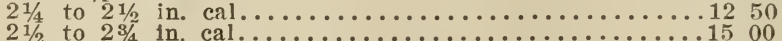

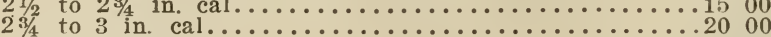

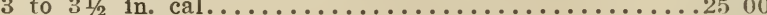

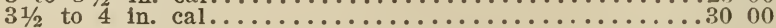

1700

2000

$\begin{array}{ll}25 & 00 \\ 35 & 00\end{array}$

5000

7500

10000

15000 
"fastigiata, Lombardy Poplar

Per 10 Per 100 Per 1000

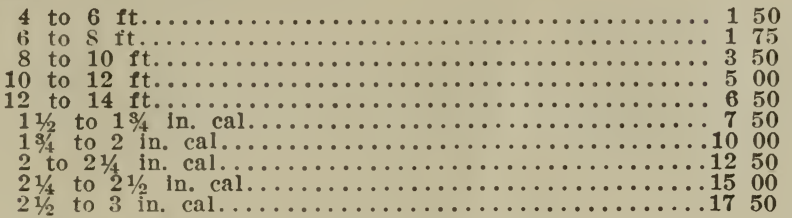

1200

1500

3500

5000

6500

These Lombardy Poplars have been several

times transplanted and are branched to the ground, but as they have central stems, it is possible to trim them up if necessary.

" monilifera, Carolina Poplar

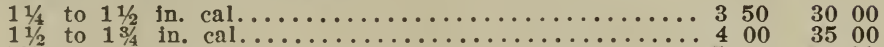

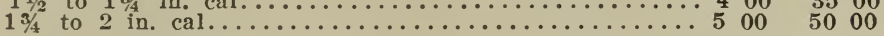

Prunus Pissardi, see shrubs

Pyrus aucuparia (see Sorbus aucuparia)

"Bechtel's Double Flowering (Ionensis)

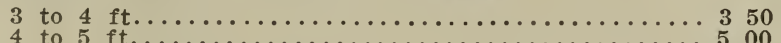

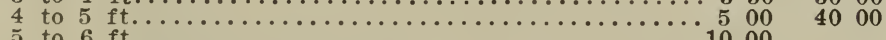

3000

" coronaria, Sweet Scented Crab

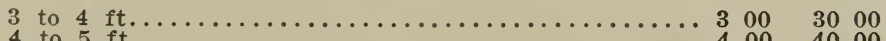

"floribunda

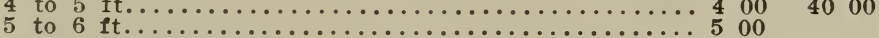

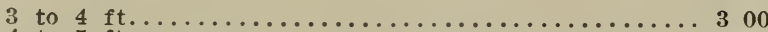

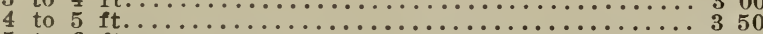

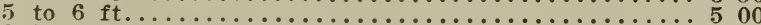

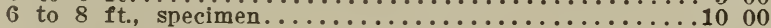

"floribunda atrosanguinea

4 to 5 ft.............................. 50

"Kiado

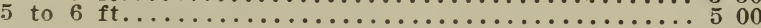

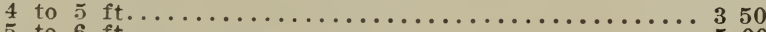

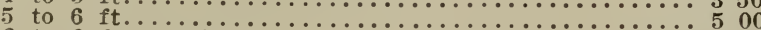

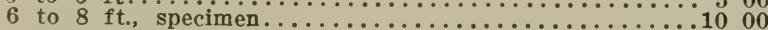

"Parkmanni-Halleana

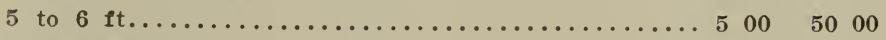

"Scheideckerii

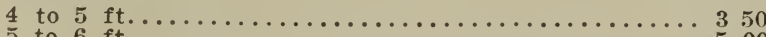

" spectabilis

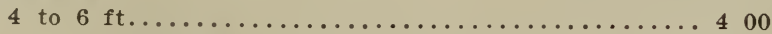

Quercus alba, White Oak

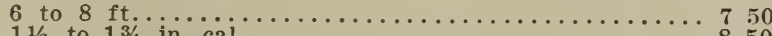

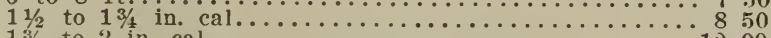

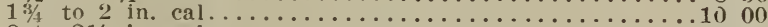

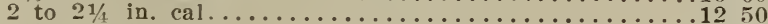

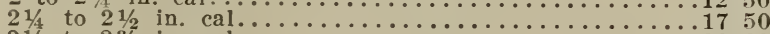

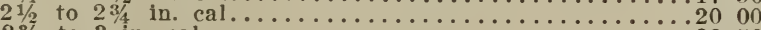

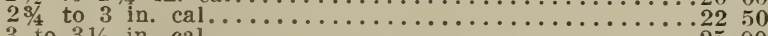

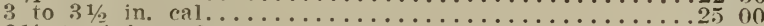

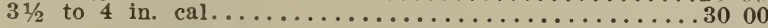

aquatica, Water Oak

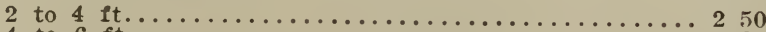

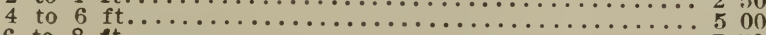

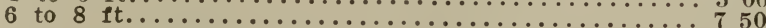

"Bannisteri, Scrub Oak

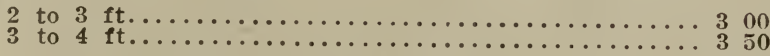

3500 
“ bicolor, Swamp White Oak

Per 10 Per $10 n$ Per 1000

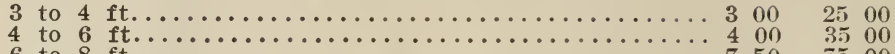

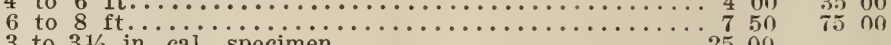

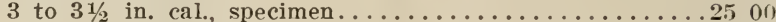

$31 / 2$ to 4 in. cal., specimen.......................

" cerris, Turkey Oak

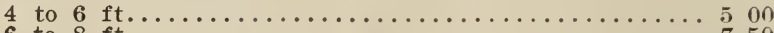

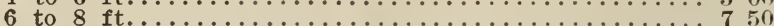

" coccinea, Scarlet Oak

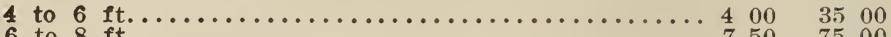

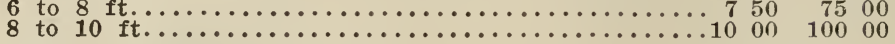

" laurifolia, Laurel-leaved Oak

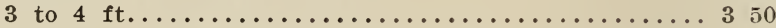

" macrocarpa, Mossy Cup or Burr Oak

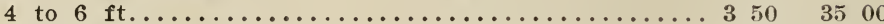

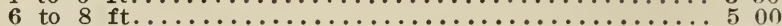

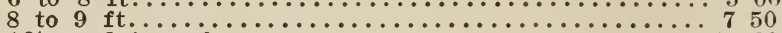

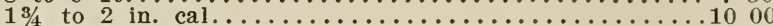

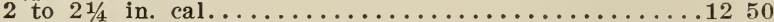

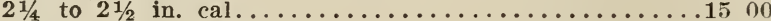

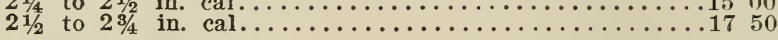

“ obtusiloba, Post Oak

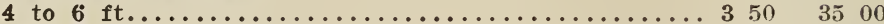

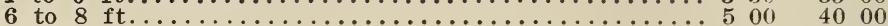

“palustris, Pin Oak

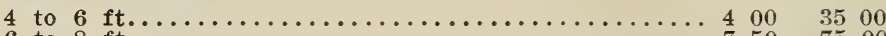

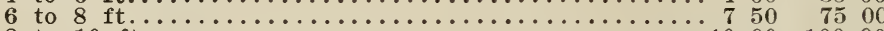

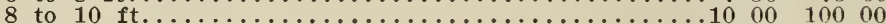

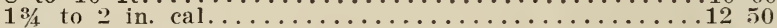

2 to $21 / 2$ in. cal. . . . . . . . . . . .

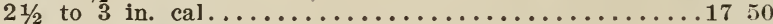

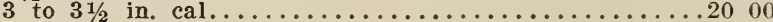

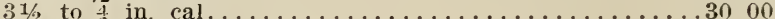

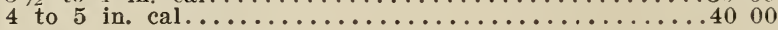

4000

"Phellos, Willow Oak

$11 / 4$ to $11 \%$ in. cal..................... 6 006000

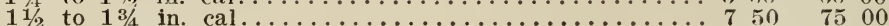

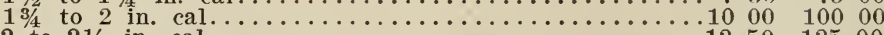

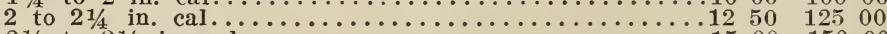

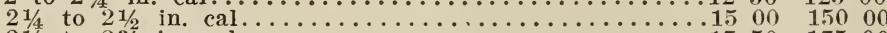

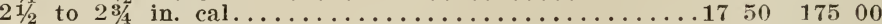

$23 / 4$ to 3 in. cal.

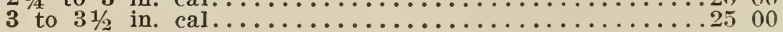

"Prinus, Rock Chestnut Oak

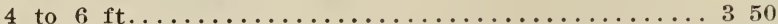

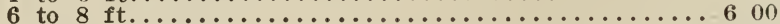

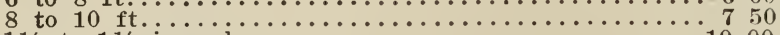

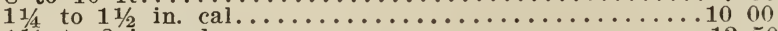

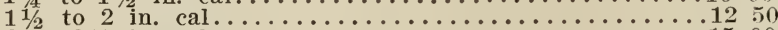

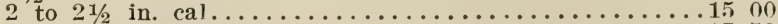

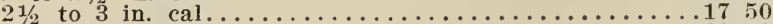

" robur, English Oak

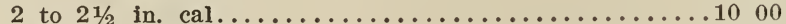

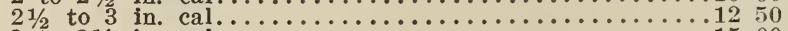

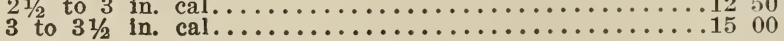

" robur pyramidalis, Pyramidal English Oak

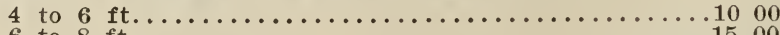

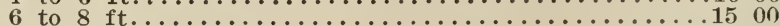

"robur concordia, Golden Oak

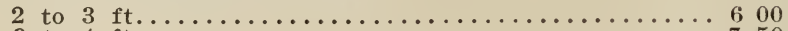

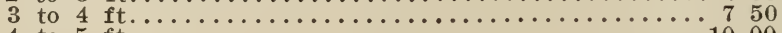

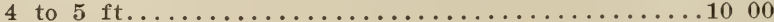


- rubra, Red Oak

Per 10 Per 100 Per 1000

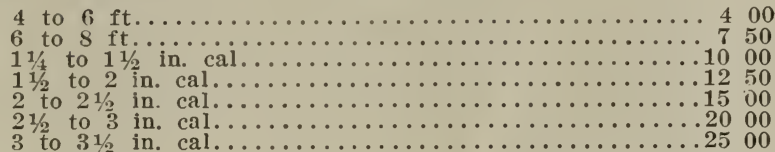

"Texana

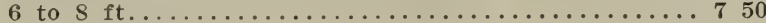

" tinctoria, Black Oak

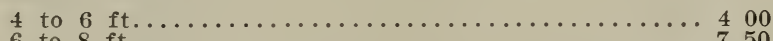

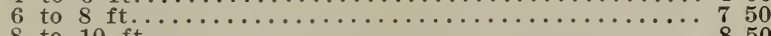

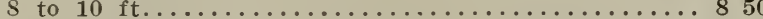

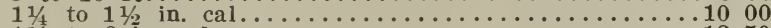

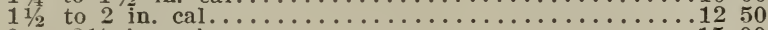

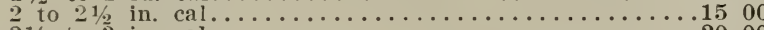

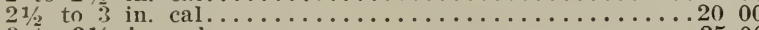

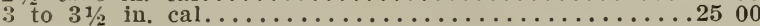

3500

7000

Robinia pseud-acacia, Yellow or Black Locust

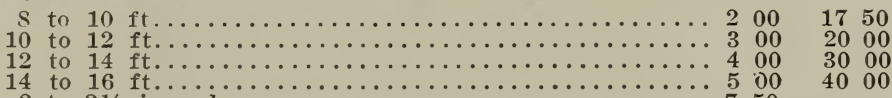

Salisburia adiantifolia, Ginkgo or Maiden Hair

6 to $s$ ft......................... 400

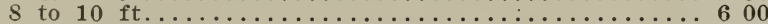

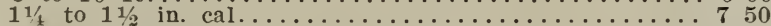

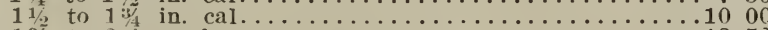

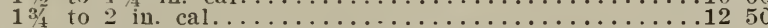

Salix aurea pendula, Golden-barked Weeping Willow

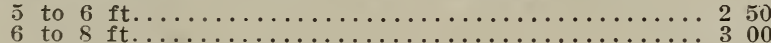

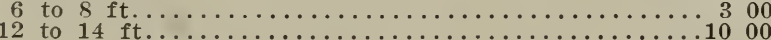

2500

" pentandra, Laurel-leaved Willow

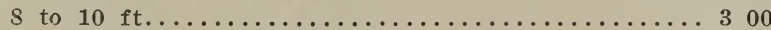

" regalis, Royal or White-leaved

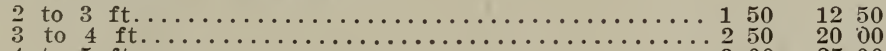

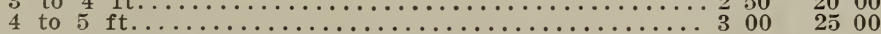

"Rosmarinifolia, Rosmary Willow

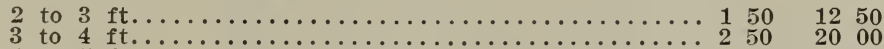

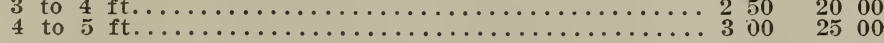

"vitellina, Golden Bark

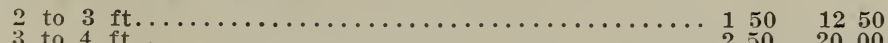

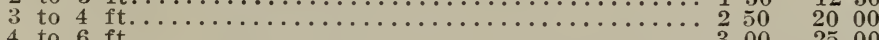

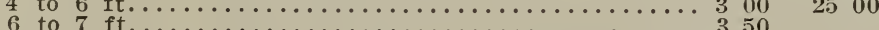

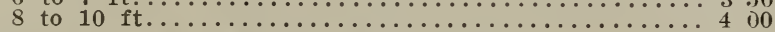

"vitellina Britzensis, Red-barked Willow

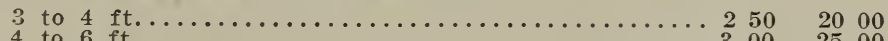

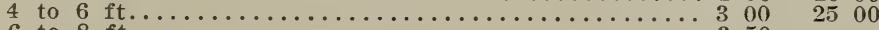

"Wisconsin, Weeping

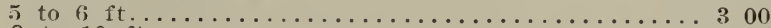

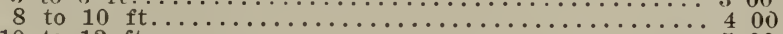

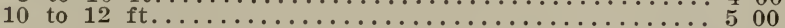

3500

Sophora Japonica

3 to 4 ft................................ $00 \quad 2500$

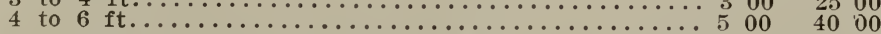

Sorbus aucuparia, European Mountain Ash

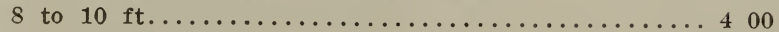


Taxodium distichum, Bald or Deciduous Cypress

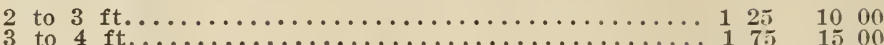

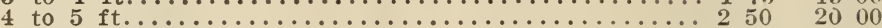

Tilia Americana, American Linden

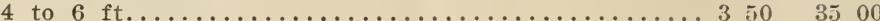

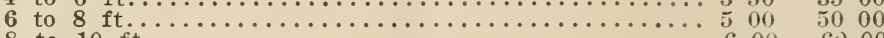

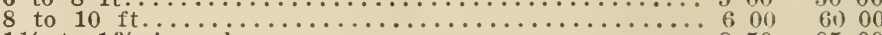

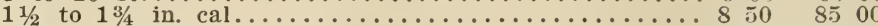

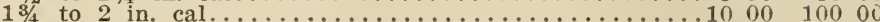

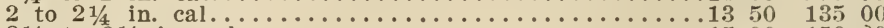

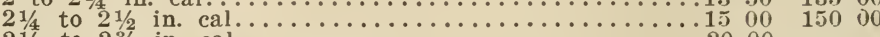

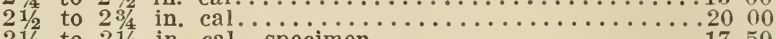

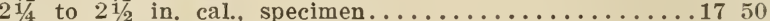

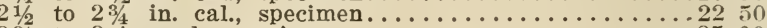

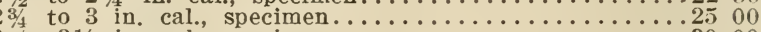

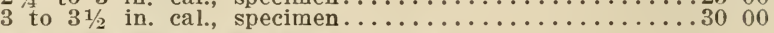

" alba argentea, Silver-leaved Linden

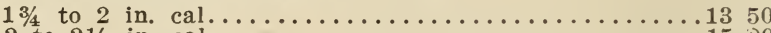

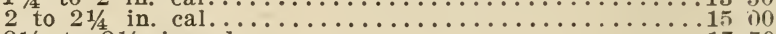

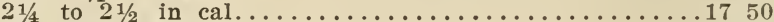

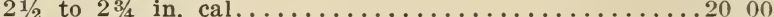

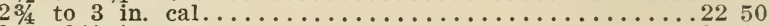

3 to $31 / 2$ in. cal. . . . . . . . . . . . . . . . .

" alba pendula, Weeping Linden

5 to $6 \mathrm{ft}$, stem, 2 year heary heads................ 00

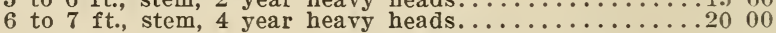

" dasystyla, Crymean or Yellow twigged Linden

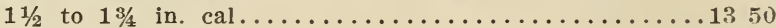

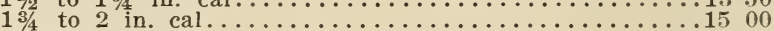

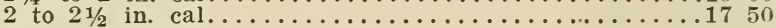

"Europaea, Small-leaved Linden

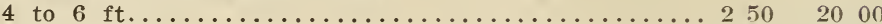

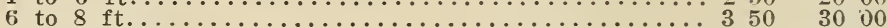

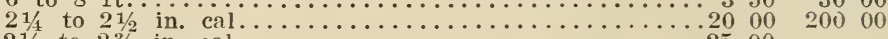

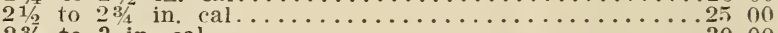

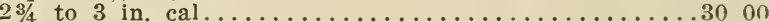

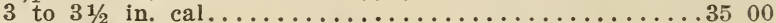

" plataphylla, Large-leaved European Linden

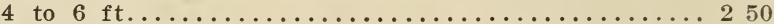

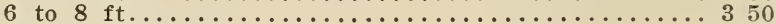

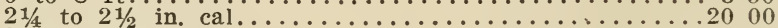

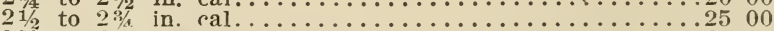

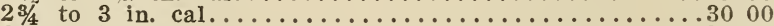

to $3 \frac{1}{2}$ in. cal. .

All of these varieties of Linden have been transplanted within the last two years. They have perfectly straight trunks and nice heads and as they have single stems right to the top of the tree, they can be trimmed higher if necessary. The dasystyla is an excellent variety for street planting, the leaves are leathery and tough and are never attacked by insects. They remain green until a hard frost, then they all fall at one time.

\section{Ulmus Americana, American EIm}

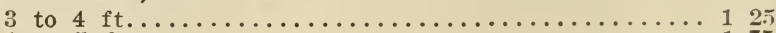

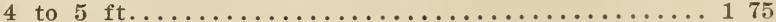

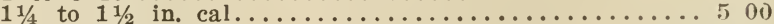

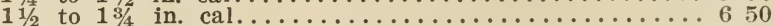

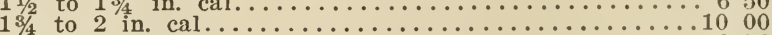

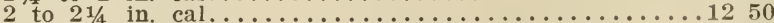

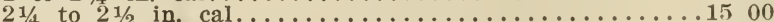

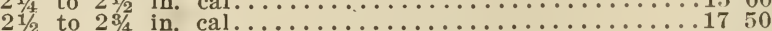

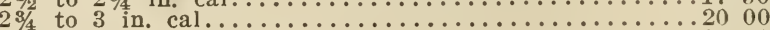

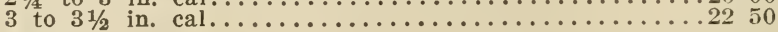

"Camperdown pendula

2 year heads............................. 600

"latifolia, Scotch Elm

$21 / 2$ to 3 in. cal.................................... 00

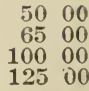

6500

12500 


\section{Deciduous Shrubs}

We continually try to impress upon our customers the fact that the mere height of a shrub does not indicate its value, but that its "bushiness" must also be taken into consideration. Shrubs grown closely in nursery rows must necessarily make height but the "bushiness" is sacrificed. A good, bushy, two to three-feet shrub is worth as much as a "slim" three to four-feet plant and for results it is worth more.

We grow our shrubs in wide rows and allow plenty of space between the plants in the rows to permit them to develop properly. It costs more to grow shrubs this way than where they are grown closely together, as we cannot plant as many on an acre. In comparing our prices with those of other growers, this fact should be given consideration.

"Heavy" shrubs indicate those which have been given extra room in the nursery rows.

Abelia rupestris

Per 10 Per 100 Per 1000

2 ft. blishy. 500

Amelanchier Boryapium

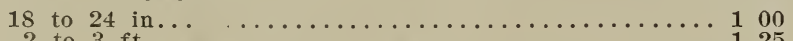

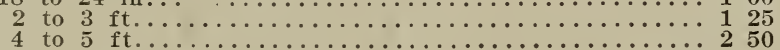

"Canadensis

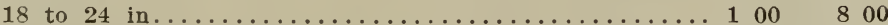

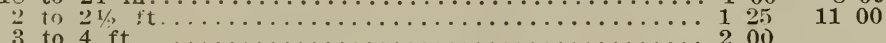

Amorpha canescens

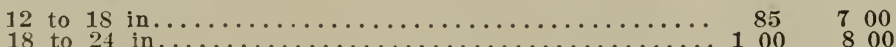

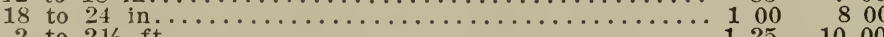

"fruticosa

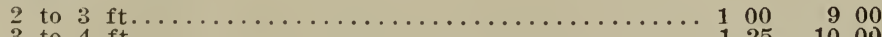

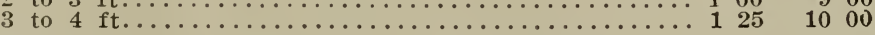

Amygdalus nana, Double White Flowering Almond

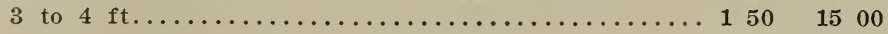

" nana, Double Pink Flowering Almond

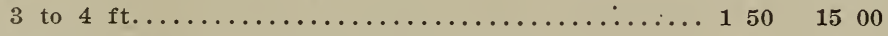

"Persica, Double White Flowering Peach

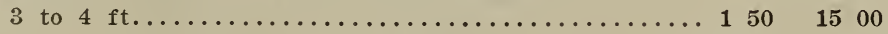

"Persica, Double Red Flowering Peach

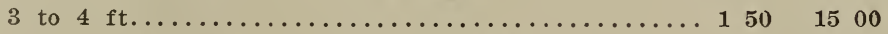

Andromeda arborea (See Oxydendron, trees)

Aralia Japonica

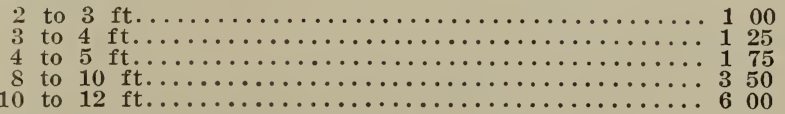

"Mandschurica (Dimorphanthus)

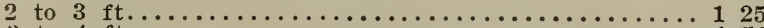

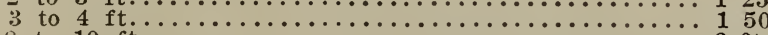

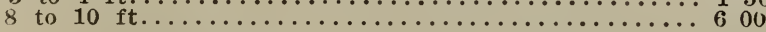


“ pentaphylla

Per 10 Per 100 Per 1000

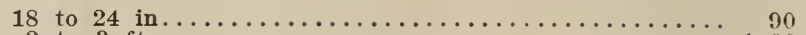

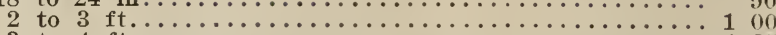

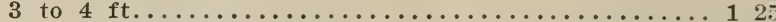

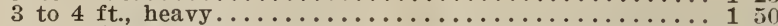

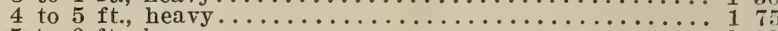

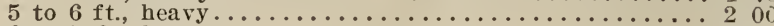

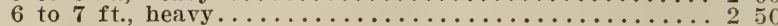

" spinosa

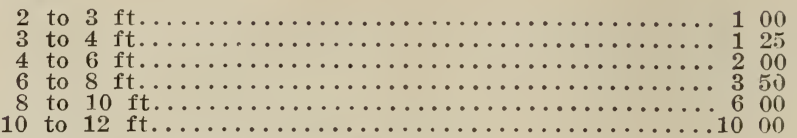

Azalea amoena, Evergreen Azalea

18 to 24 in., 5 in. pots.................... 400

" amoena Hinondigiro, Bright Carmine

12 to 15 in., bushy 6 in. pots...................... 00

" arborescens

$2 \mathrm{ft}$, bushy.......................4 400

“ Mollis, Red

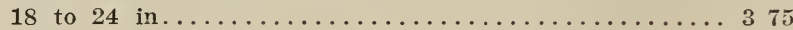

" Mollis, Yellow

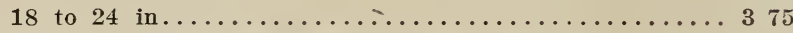

"Pontica (Ghent), assorted colors

18 to 24 in........................... 00

“Vaseyii

12 to 18 in, bushy.......................... 00

Baccharis halimifolia, Groundsel Shrub

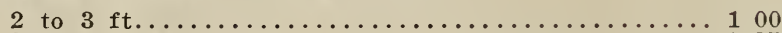

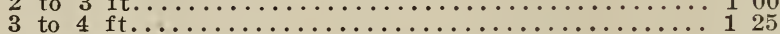

Berberis ilicifolia, Holly-leaved Barberry

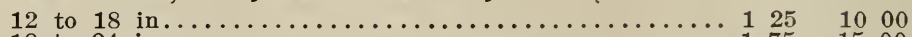

“ purpurea, Purple Barberry

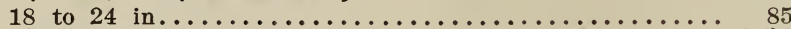

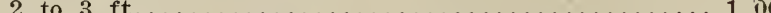

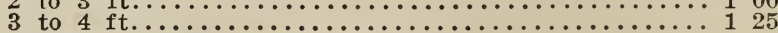

“ Thunbergii, Japanese Barberry

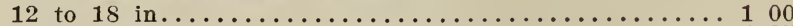

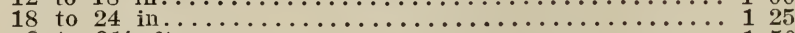

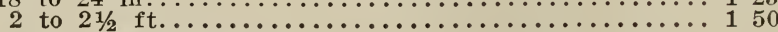

800

1000

1200

1500

1800

“ vulgaris, Green Barberry

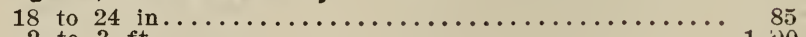

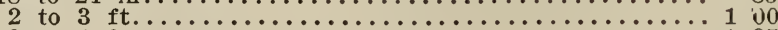

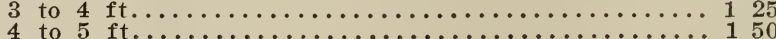

Buddleia intermedia

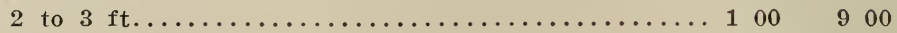

Callicarpa purpurea

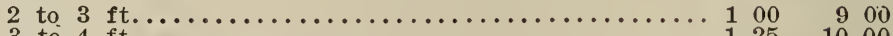

Calycanthus floridus, Sweet Shrub

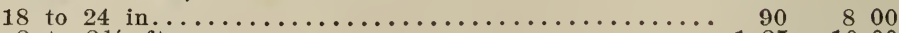

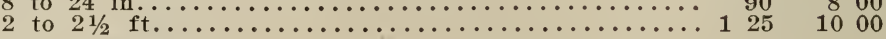

Caragana arborescens

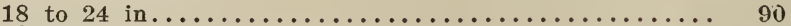

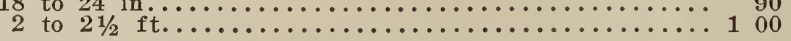

" arborescens pendula, Weeping Siberian Pea

5 to $6 \mathrm{ft}$, stems, 3 year heads................. 850

6 to 8 ft., stems, 3 year heads...................... 
Per 10 Per 100 Per 1000

Carpinus Americana, American Hornbeam

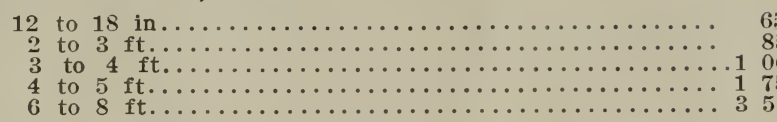

$\begin{array}{rlll}4 & 00 & 30 & 00 \\ 5 & 00 & 40 & 00 \\ 8 & 00 & 70 & 00\end{array}$

"Betulus, European Hornbeam

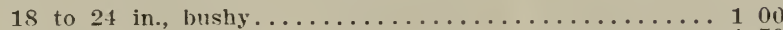

2 to 3 ft., bushy.................... 150

3 to $4 \mathrm{ft}$. bushy...................... 175

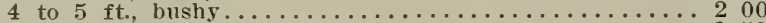

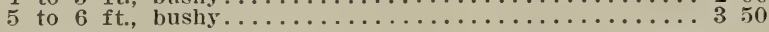

Both the American and European Hornbeam make excellent hedges. The plants offered are grown especially for this purpose.

Caryopteris Mastacanthus, "Blue Spiraea"

18 in., bushy....................... 00

Ceanothus Americana, New Jersey Tea

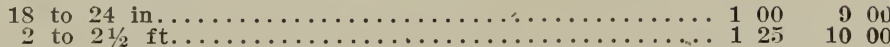

Cephalanthus occidentalis, Dwarf Button-bush

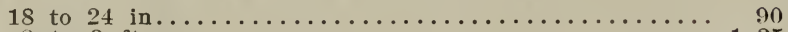

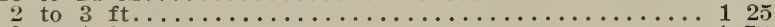

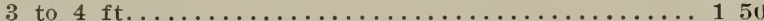

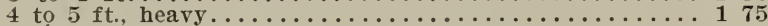

800

1000

1500

9000

Cercis Canadensis, American Judas or Red Bud

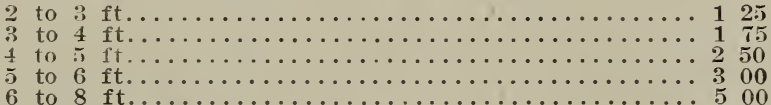

Chionanthus Virginica, White Fringe

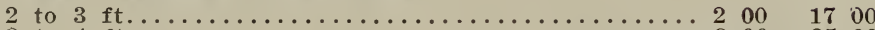

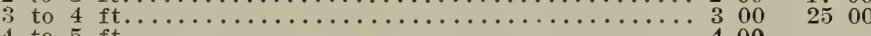

Clethra alnifolia, Sweet Pepper Bush

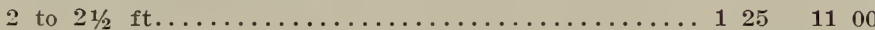

Colutea arborescens

2 to $21 / 2$ ft.......................... $100 \quad 800$

Corchorus, see Kerria

Cornus alba (Siberica), Red-twigged Dogwood

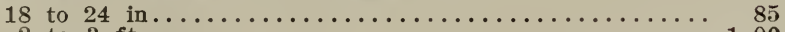

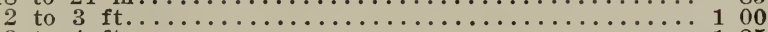

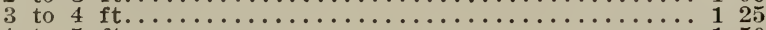

800

1000

1200

1100

1500

2000

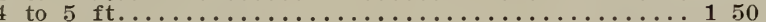

" florida, White Flowered Dogwood (see trees)

“ florida rubra, Red Flowered Dogwood (see trees)

" glabrata

4 to $5 \mathrm{ft}$., heavy....................... 150

"Mas. Cornelian Cherry

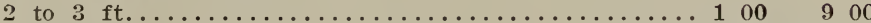

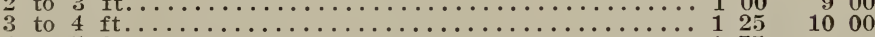

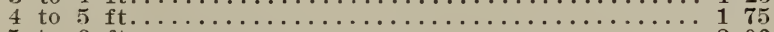

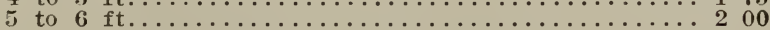

700 $\begin{array}{rr}9 & 00 \\ 10 & 00\end{array}$ 1200

"paniculata

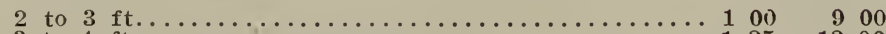

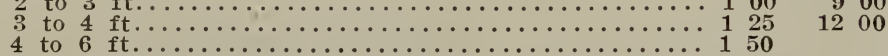

"sanguinea, English Dogwood

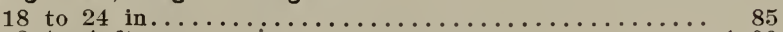

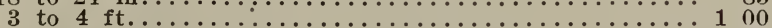

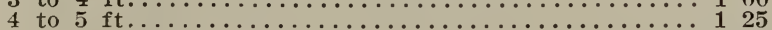

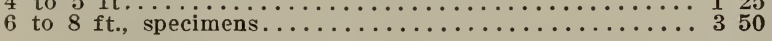


"s sericea (Amomum) Silky Dogwood

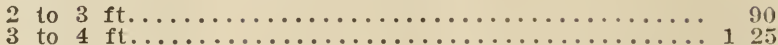

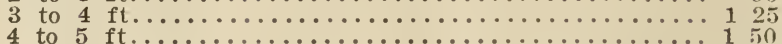

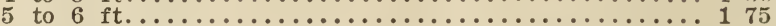

“ stolonifera lutea (flaviramea), Yellow-barked

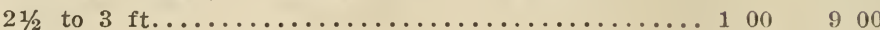

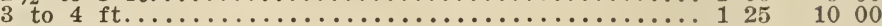

Corylus Americana, American Hazel

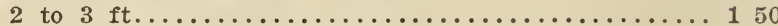

" avellena, European Hazel

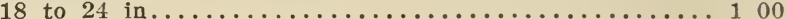

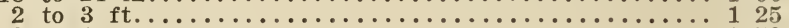

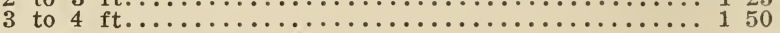

" purpurea, Purple-leaved Filbert

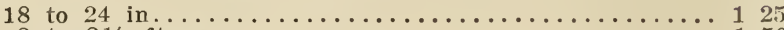

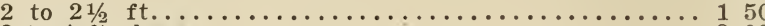

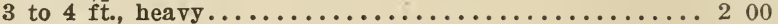

Crataegus coccinea, White Thorn

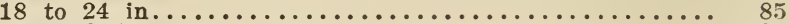

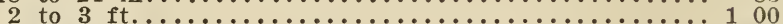

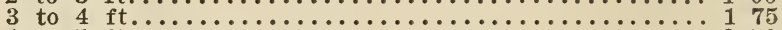

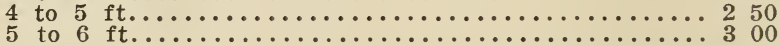

* Carriere

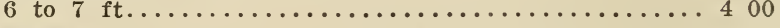

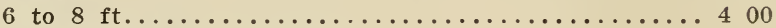

"Cordata, Washington Thorn

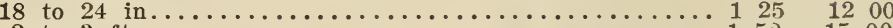

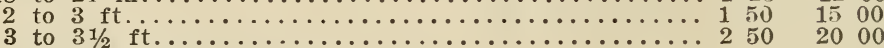

4 crus galli, Cockspur Thorn

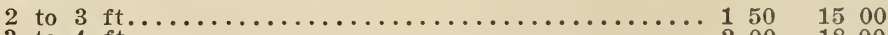

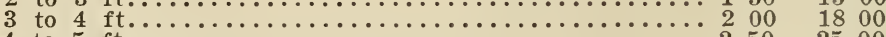

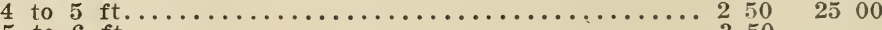

"Mollis

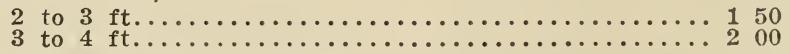

"oxycantha, English Hawthorn

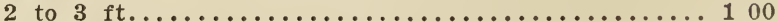

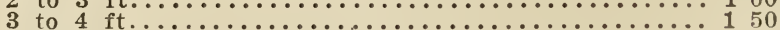

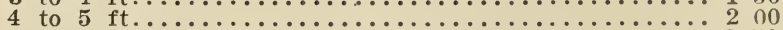

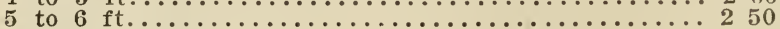

" oxycantha, Double Pink

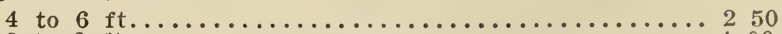

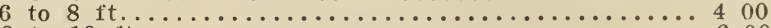

2500

8 to $10 \mathrm{ft} . \ldots . \ldots 606$

* oxycantha, Paul's Scarlet

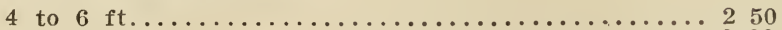

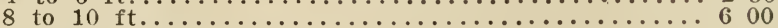

2500

"oxycantha, Double White

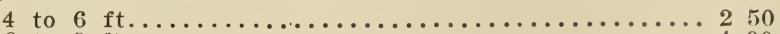

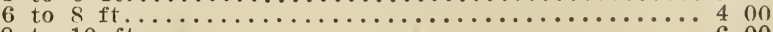

“ spathulata

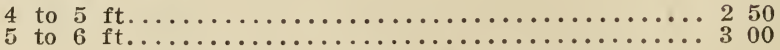

Cydonia Japonica (Pyrus Japonica), Japanese Quince

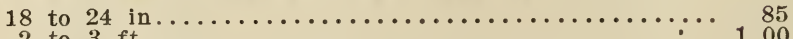

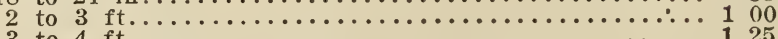

"Moerloosei, Pink

2 to $3 \mathrm{ft}$ 
" umbillicata, Bright Rose

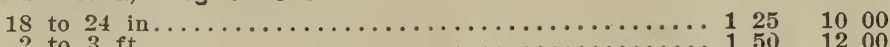

\section{Cytissus Laburnum}

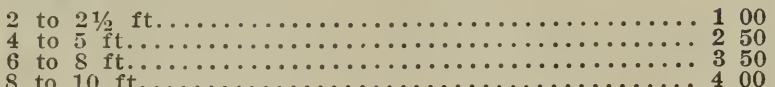

\section{Daphne Mezereum album}

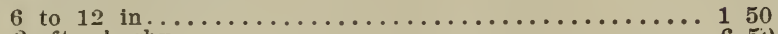

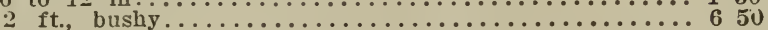

" Mezereum rubrum, Pink

18 to 24 in., bushy....................... 650

Desmodium penduliflorum, Pink

2 year .............................. 1251000

Deutzia candidissima plena, Double White

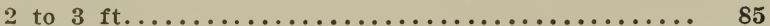

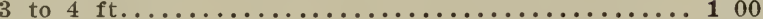

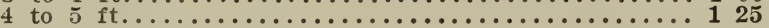

900

1000

" crenata, Double White

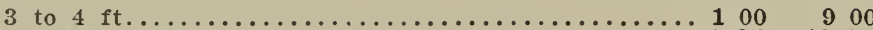

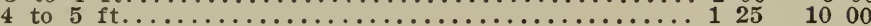

" crenata, Double Pink

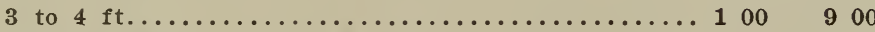

" crenata, Single White

5 to $7 \mathrm{ft}$, bushy specimens.......................

"Fortuneii, White

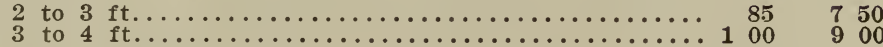

" gracilis, Dwarf White

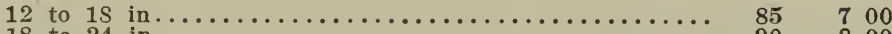

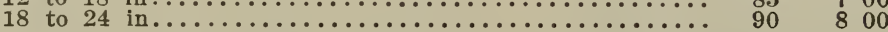

"Lemoinei, Dwarf White

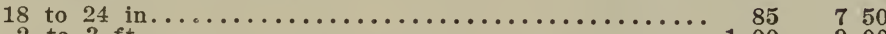

"Pride of Rochester, Double Pink

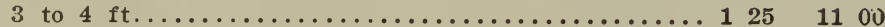

Diervilla sessilifolia (lutea)

18 to 24 in............................ 00 . 800

Dimorphanthus (see Aralia Mandschurica)

Eleagnus angustifolia, Russian Olive

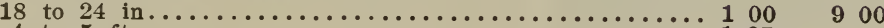

" longipes

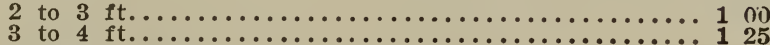

"U umbellatus, female

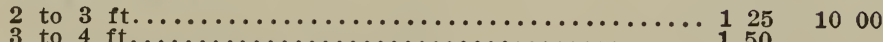

Euonymus alatus, Japanese Euonymus

18 to 24 in., bushy..................... 50

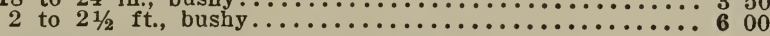

"Europaeus, European Burning Bush

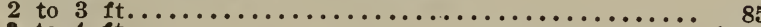

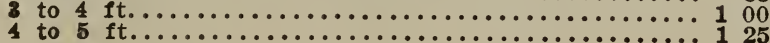


Exochorda grandiflora, Pearl Bush

Per 10 Per 100 Per 1000

2 to 3 ft........................

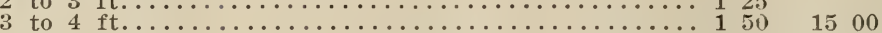

Forsythea Fortunei, Golden Bell

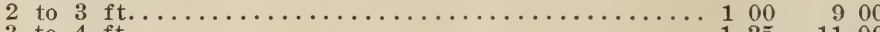

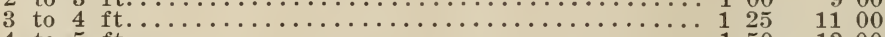

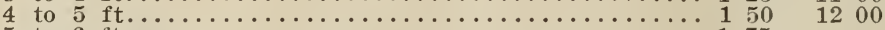

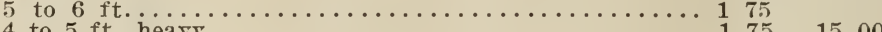

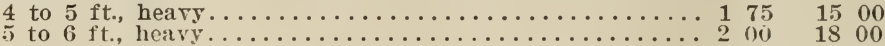

" suspensa

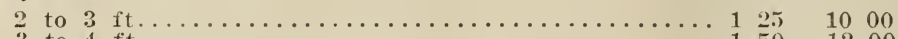

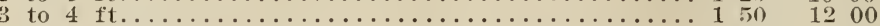

" viridissima

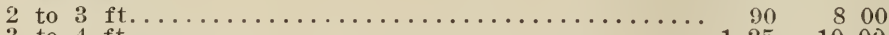

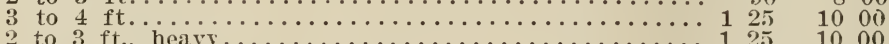

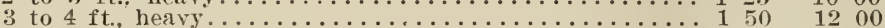

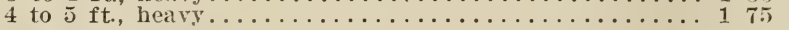

" viridissima variegata

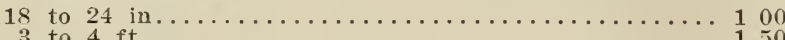

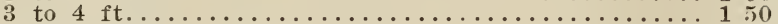

Halesia tetraptera, Silver Bell

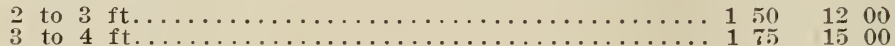

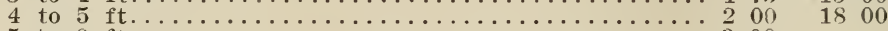

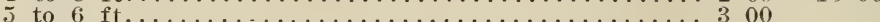

Hamamelis Virginica, Wych-hazel

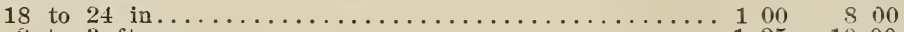

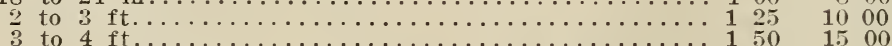

HIBISCUS MEEHANI VARIEGATA

New Single Fiowered Variegated Leaved Althaea.

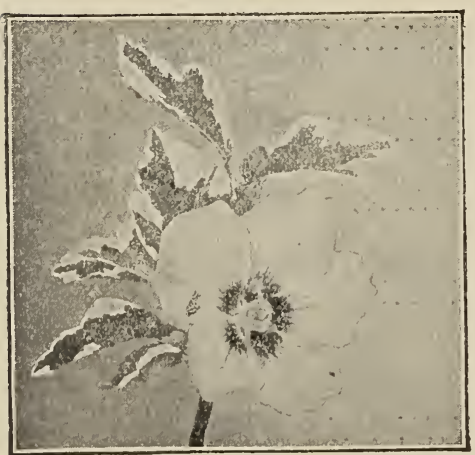

This new variety of Althæa hás proven very attractive since we first offered it two years ago. The leaves are variegated with green and white just the same as the old double flowered sorts, but its great beauty lies in the fact that the flowers, those single, open wide, being no less than four inches in diameter, while the flowers of the old double sort do not open at all. The color is a lilac pink. For prices see list of Hibiscus.

Hibiscus alba piena, Double White Althaea

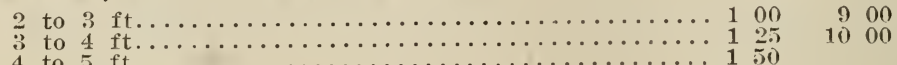

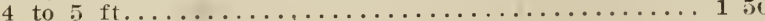

900

“ ardens, Double Blue

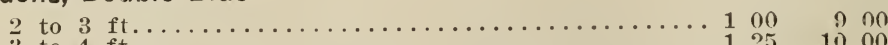
ft..........................

" bicolor, Double Pink

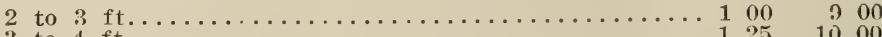

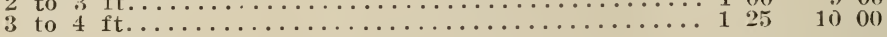


Per 10 Per 100 Per 1000

" Boule de Feu, Double Red

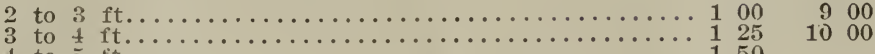

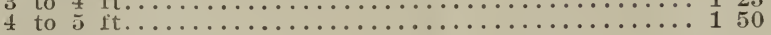

" coerulea, Double Blue

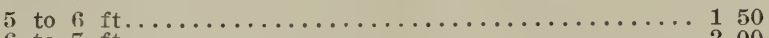

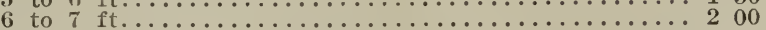

"Duc de Brabant, Double Red

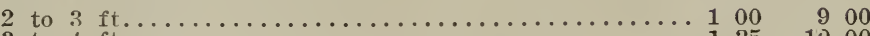

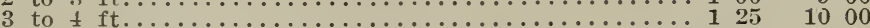

" elegantissima, Double, delicate pale pink

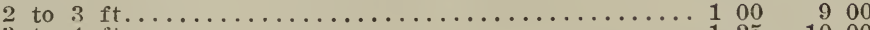

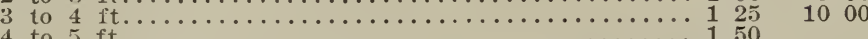

"Jean d'Arc, Double White

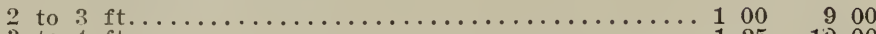

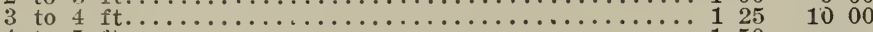

"Lady Stan!ey, Double Pink

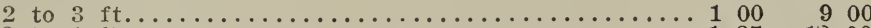

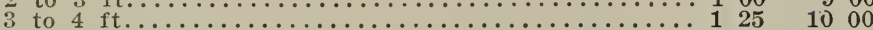

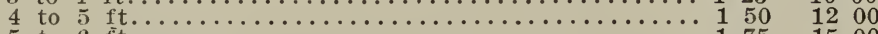

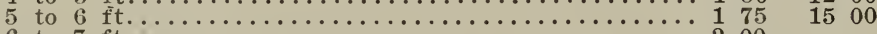

" variegata fl. pl., Variegated-leaved, double red

18 to 24 in .................................. 00

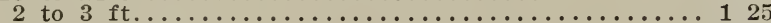

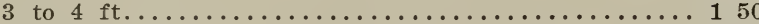

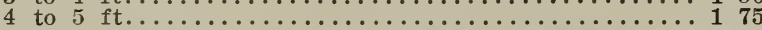

Meehani variegated, Single flowered variegated-leaved

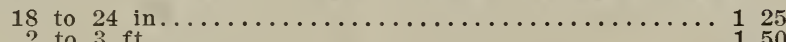

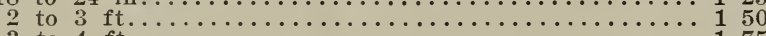

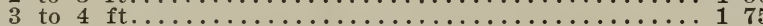

"totus alba

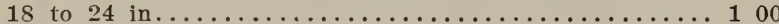

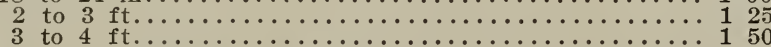

900

1000

1200

1500

1000

1200

900

1000

Hippophaea rhamnoides, Sea Buckthorn

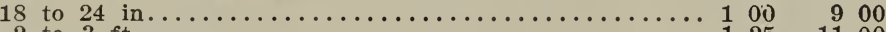

\section{Hydrangea arborescens}

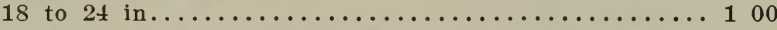

" arborescens grandiflora (new) American Everblooming

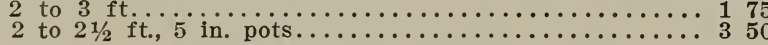

“ paniculata (type), Single

2 to 3 ft.......................... $100 \quad 900$

" paniculata acuminata, Early Flowering (type), Single

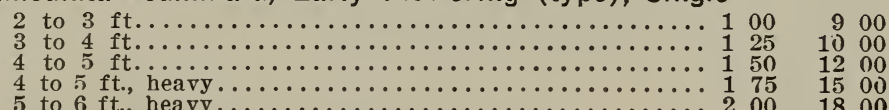

" paniculata grandiflora

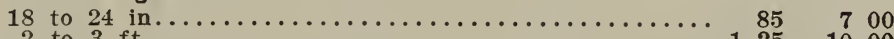

“ paniculata grandiflora Standards

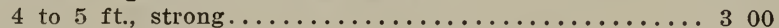

" quercifolia, Oak-leaved

Hydrangea

1500

1000

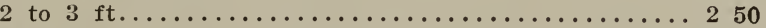

" radiata

18 to 24 in ............................. 100 900

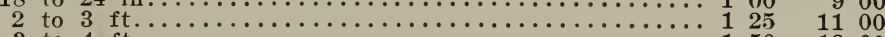

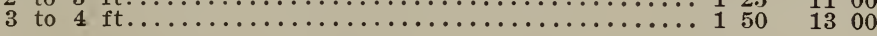




\section{Hypericum aureum}

" densiflorum

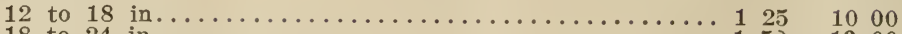

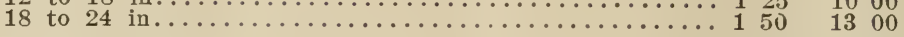

"Moserianum

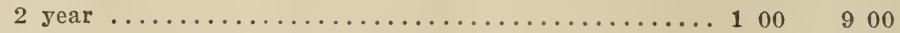

"prolificum

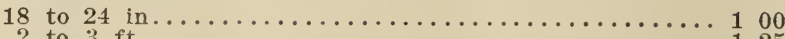

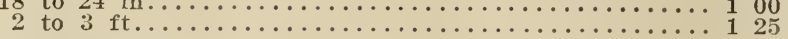

llex verticillata (Prinos)

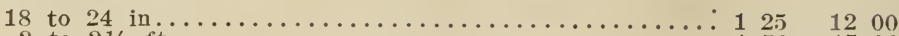

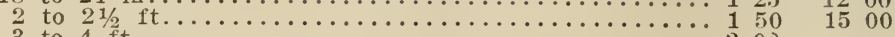

" decidua, Deciduous Holly

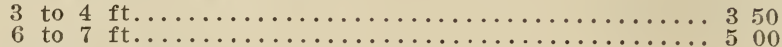

\section{Itea Virginica}

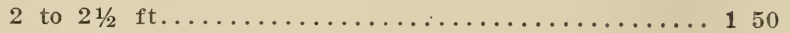

1300

Kerria Japonica, double (Corchorus)

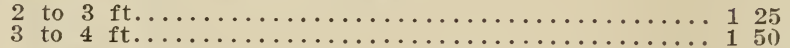

" Japonica, Single

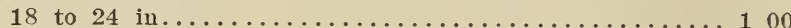

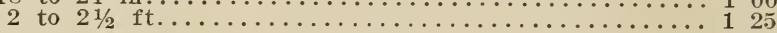

"Japonica variegata

18 to 24 in............................... 125

Laurus Benzoin (see Lindera)

Lespedeza bicolor

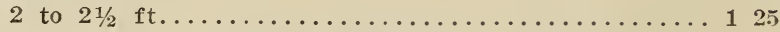

Ligustrum ibota, as commonly sold, not upright

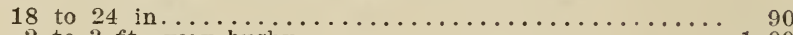

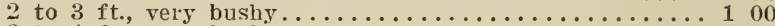

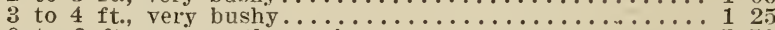

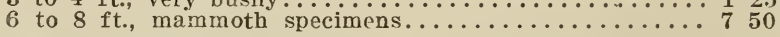

"ovalifolium, Californa Privet

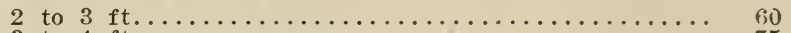

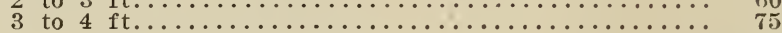

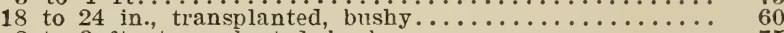

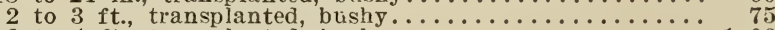

3 to 4 ft., transplanted, bushy................... 100

4 to $5 \mathrm{ft}$., transplanted, bushy................ 125

The several grades of California Privet offered as "transplanted, bushy" are very fine plants. They have been actually transplanted in the nursery, they are nice bushy plants and heavily rooted. For planting for a hedge for immediate effect this transplanted stock will give quicker results than the regular nursery grades.

“ Regelianum

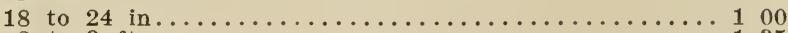

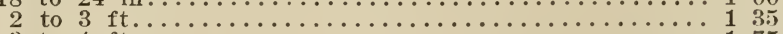

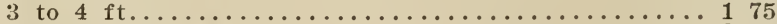

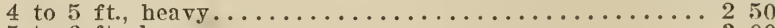

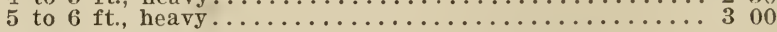

" vulgaris, Common Privet

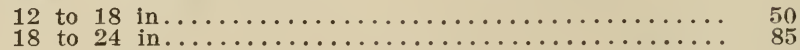

900

1200

1500

2000

2500

2500

3000

$\begin{array}{lll}40 & 00\end{array}$

50 00

6000 
Per 10 Per 100 Per 1000

\section{Lonicera Bella albida}

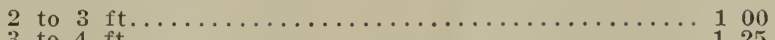

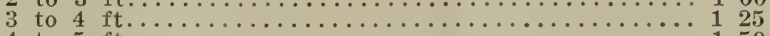

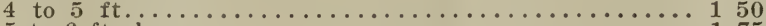

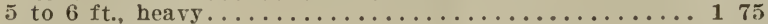

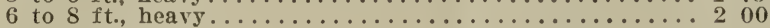

" bella candida

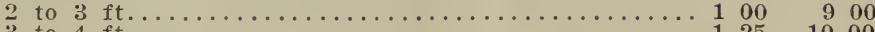

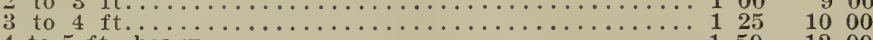

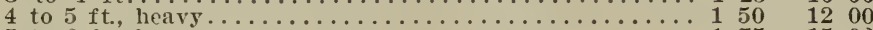

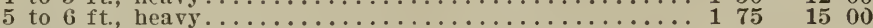

"Ledebouri,

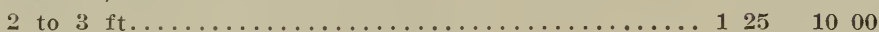

“ Morrowii

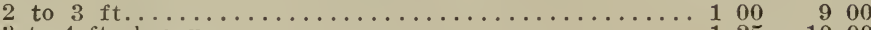

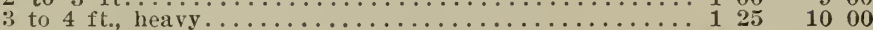

"Orientalls, White

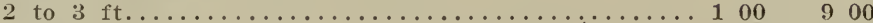

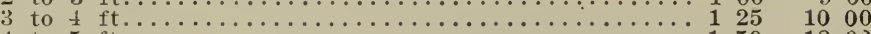

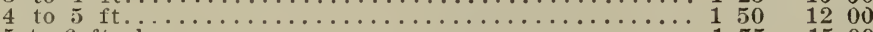

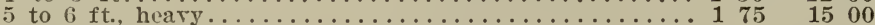

"Ruprechtiana

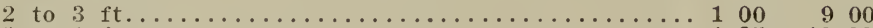

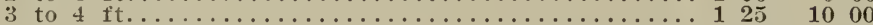

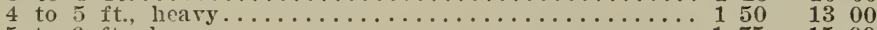

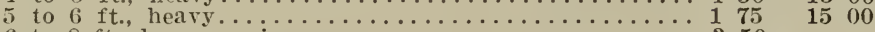

6 to $8 \mathrm{ft}$., large specimens.............................

Tatarica alba

18 to 24 in........................... $85 \quad 750$

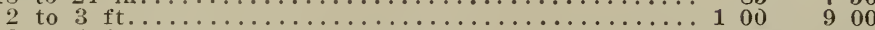

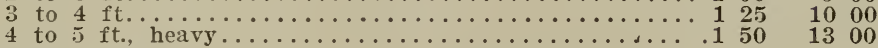

"Tatarica, Red

3 to $4 \mathrm{ft}$, heary...................... $150 \quad 1300$

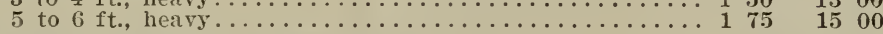

"Tatarica grandiflora rosea, Pink

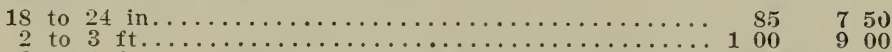

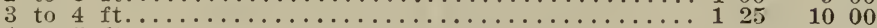

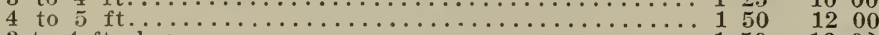

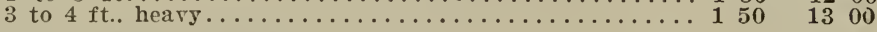

“ Tatarica Yellow Berried

3 to $4 \mathrm{ft}$, heavy..................... 1501300

"Zylosteum

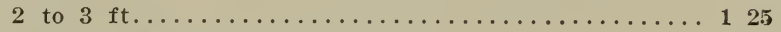

Oxydendron arboreum, Sorrel Tree

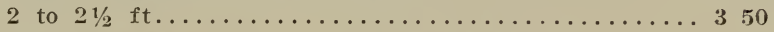

Philadelphus aureus (Syringa) Golden-leaved

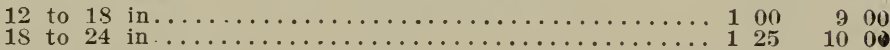

“ coronarius, Sweet Flowered Mock Orange

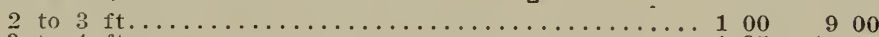

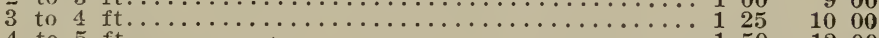

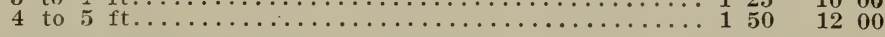

" grandiflorus, Large Flowered Mock Orange

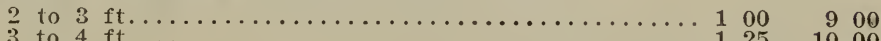

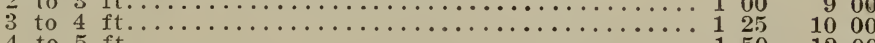

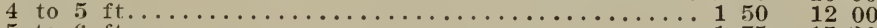

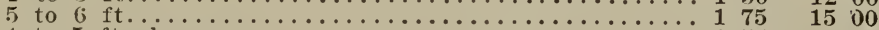

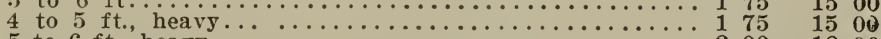

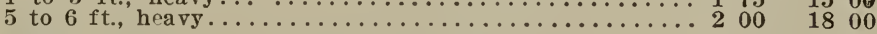

“ Lemoinei

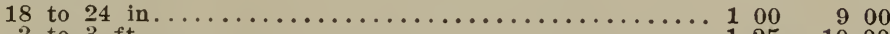

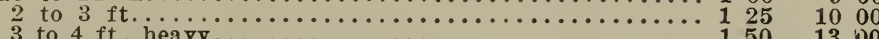

3 to $4 \mathrm{ft}$, heary....................... 15013 vo 
“ maculata purpurea, New. Pink

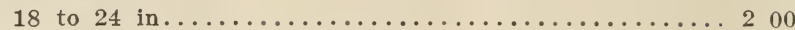

“ nanus, Dwarf Mock Orange

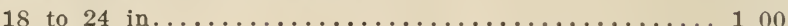

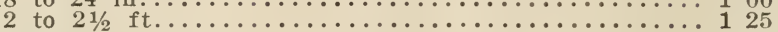

900

"Zeyherii

3 to 4
4
4
5 to $\begin{aligned} & 5 \\ & 6\end{aligned}$

Photinia villosa

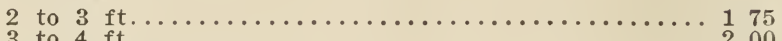

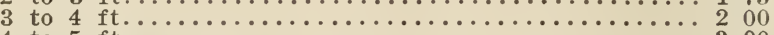

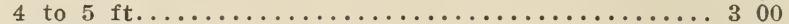

Potentilla fruticosa

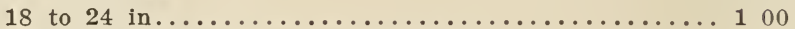

900

Prinos verticillata (See llex verticillata)

Prunus Japonica (See Amygdalus nana)

“ Maritima, Beach Plum

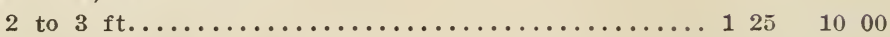

“Pissardi, Purple-leaved Plum

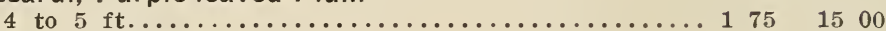

" triloba, dbl. fl. Plum

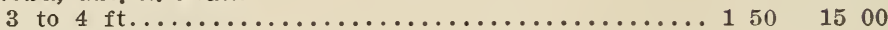

Ptelea trifoliata, Hop Tree

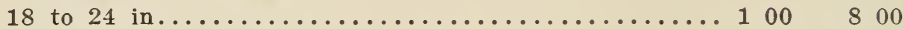

Pyrus Japonica (see Cydonia Japonica)

" arbutifolia

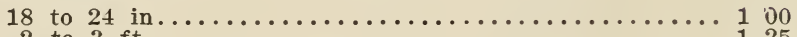

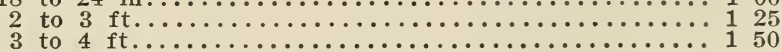

"Bechtels, and other large growing sorts (see Trees)

“ melanocarpa

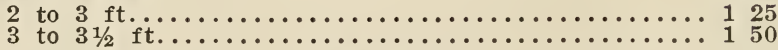

Rhamnus catharticus, Common Buckthorn

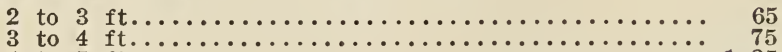

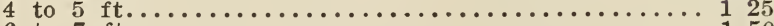

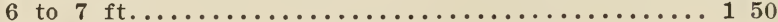

"frangula

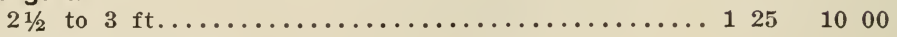

Rhodotyphus kerroides

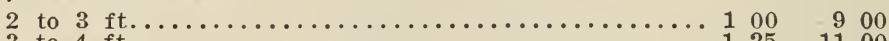

$\begin{array}{ll}5 & 50 \\ 6 & 00\end{array}$

800

4500

5000

1200

7000

Rhus aromatica, sweet Sumach

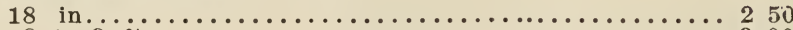

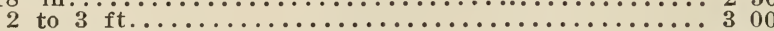

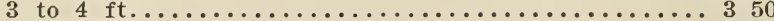

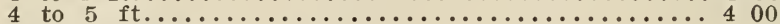

$\begin{array}{ll}22 & 00 \\ 25 & 00\end{array}$

3000

" copallina, Shining Sumach

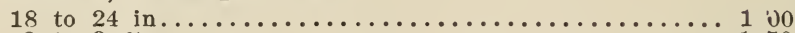

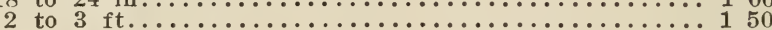

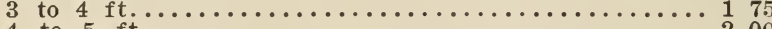

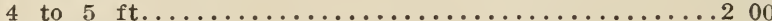

1200

1500

" cotinus, Mist or Smoke Tree

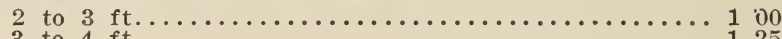

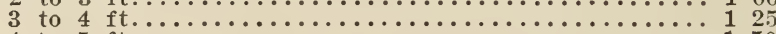

900

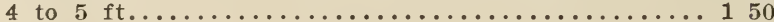


Per 10 Per 100 Per 1000

" glabra, Smooth Sumach

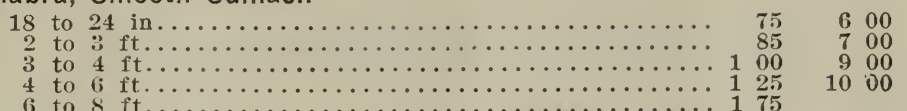

" glabra laciniata, Cut-leaved Sumach

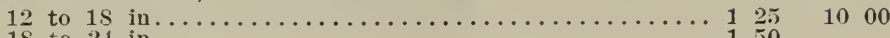

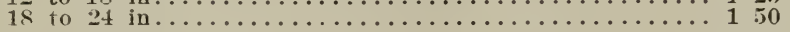

"typhina, Stag's Horn Sumach

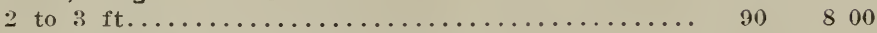

" typhina laciniata, Cut-leaved Sumach

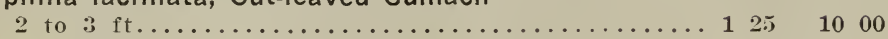

Ribes aureum, Yellow Flowering Currant

2 to $21 / 2 \mathrm{ft} \ldots \ldots \ldots \ldots \ldots \ldots \ldots \ldots \ldots \ldots \ldots \ldots \ldots \ldots$. $00 . \ldots 00$

"Floridum, Missouri Black Currant

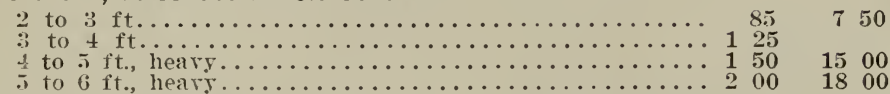

Robinia hispida rosea, Pink Rose Acacia

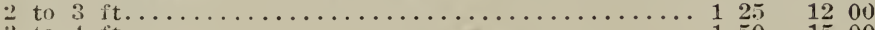

\section{Rosa blanda}

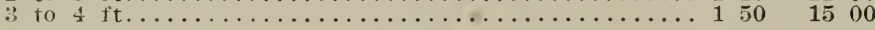

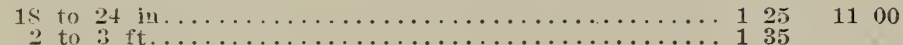

\section{Carolina}

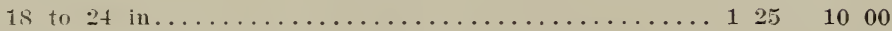

"humulis

1 is to 24 in........................... 1251000

" lucida

18 to 24 in......................... 12.51000

" multiflora Japonica

18 to 24 in........................ 251000

" nitida

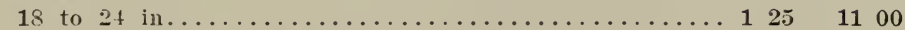

" rubiginosá, Sweet Brier

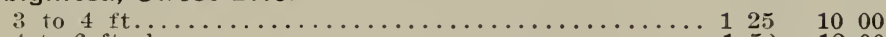

4 to $6 \mathrm{ft}$.. heary............................ 1501200

" rubrifolia

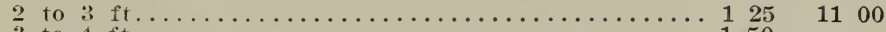

“ rugosa, Red

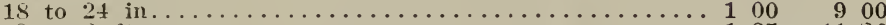

" rugosa alba, White

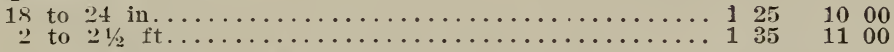

"Conrad Ferdinand Meyer

6 in. pots................................. 00

"Mme. Geo. Bruant, Double White

2 year....................... 150

" setigera, Prairis Rose

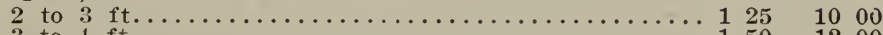

"Baby Rambler

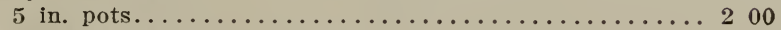

" spinossissima, Szotch Rose

.j in. pots. 


\section{Climbing and Pillar Roses}

Rose, Baltimore Belle, Double White

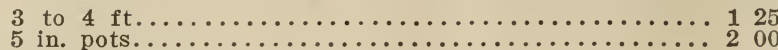

“ Crimson Rambler

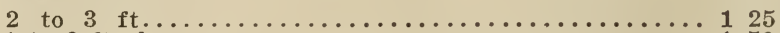

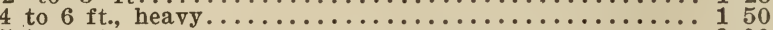

1000

“ Dawson. pink

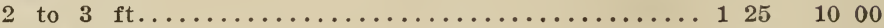

“Mad. Plantier

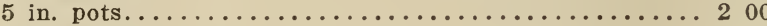

“ Pink Rambler

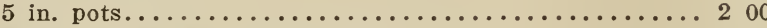

4 Queen of Prairie, Double Pink

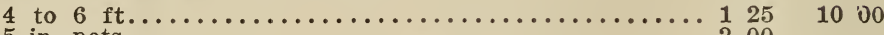

"White Rambler

2 to 3 ft.......................... 1251000

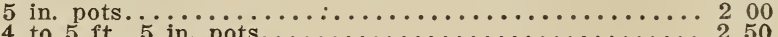

“Yellow Rambler

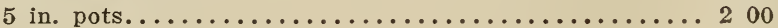

\section{Wichuraiana and Hybrids}

Rosa Wichuraiana, Memorial Rose

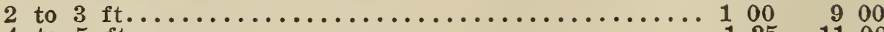

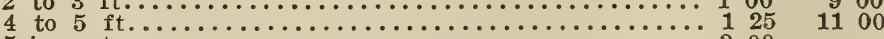

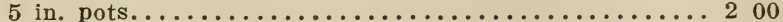

"Alberic Barbier, Pure White, Double

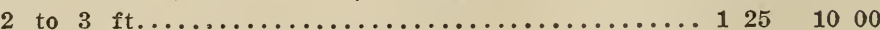

“ Dorothy Perkins, Pink, Double

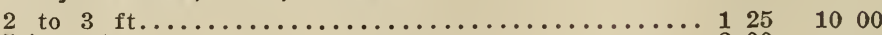

"Evergreen Gem, White, buff in bud, Double

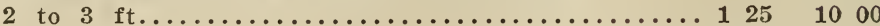

"Jersey Beauty, Buff, Yellow stamen, Single

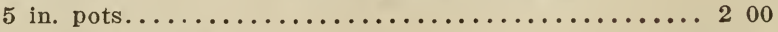

"Lady Gay, Double Pink

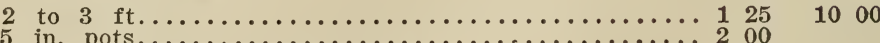

- Manda's Trumph, White, Double

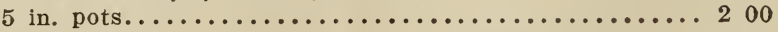

"Pink Roamer, Pink, Single

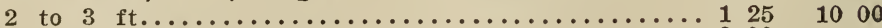

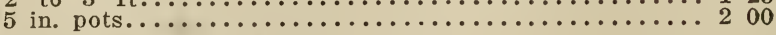

“ Rene Andre, Carmine, Semi-double

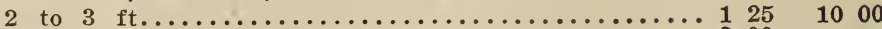

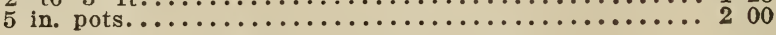

“ South Orange Perfection, White, Double

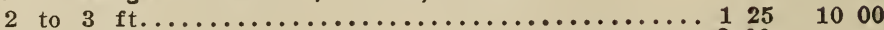

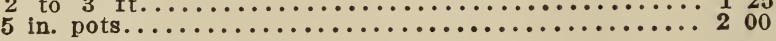

* Universal Favorite, Rose, Double

2 to 3 ft............................. 1251000

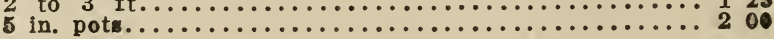




\section{Roses-Hybrid Perpetual, etc.}

Per 10 Per 100 Per 1000

Assorted varieties as follows-from 5 and 6 in. pots... 250

COQUETTE DES ALPS, flesh white

GEN. TACQUIMINO'T, brflliant crimson

GRTSS AN TEPLITZ, dark red

HERMIOSA, pink

KAISEIIN AUYUSTE VICTORIA, creamy white

LA FRANCE, silvery pink

MAGNA CHARTA, pink suffused with carmine

MAMAN COCHET, pink

MARGARET DICKSON, white, pale flesh center

MRS. JOHN LAING, soft pink

MRS. R. G. SHARMAN CRAWFORD, deep rose pink

PAUL NEYRON, deep rose

PERSIAN YELLON

PRINCE CAMILLE DE ROHAN, velvety crimson

Rubus odoratus, Flowering Raspberry

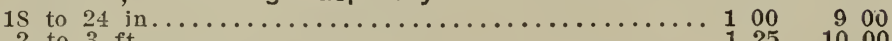

Sambucus aurea, Golden Elder

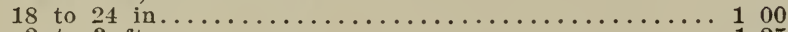

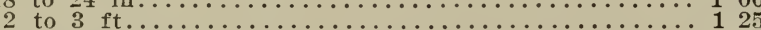

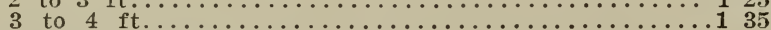

900

10. 00

1200

"Canadensis, Elderberry

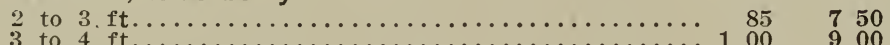

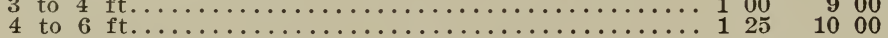

" nigra laciniata, Cut-leaved Elder

2 to $2 \frac{1}{2}$ ft................................. $00 \quad 900$

" racemosa, Red-berried Elderberry

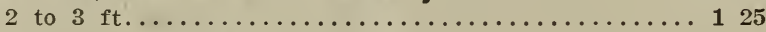

Spiraea Anthony Waterer, Pink Dwarf

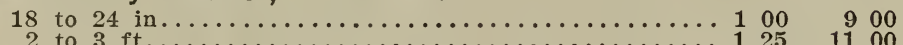

" arguta

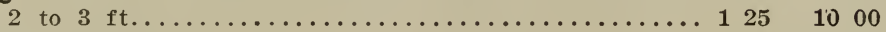

"Billardi, Pink

2 to $21 / 2$ ft................................ 85

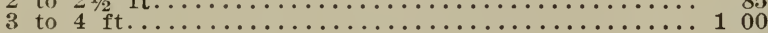

" bumalda

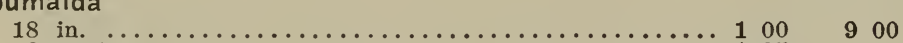

" callosa alba, Dwarf White

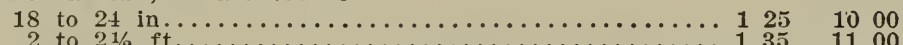

" callosa rosea, pink

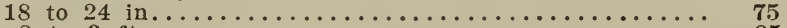

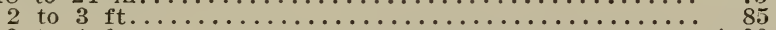

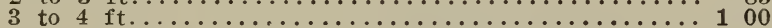

" callosa superba

2 to $21 / 2$ ft................................. 251000

“ carpinaefolia

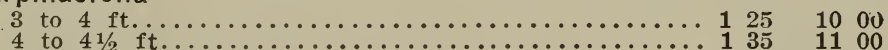

"Douglassi rosea

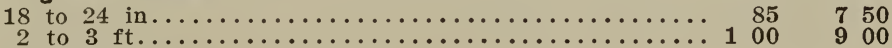

“ Lindleyana

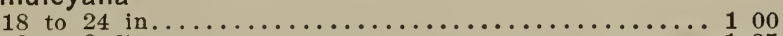

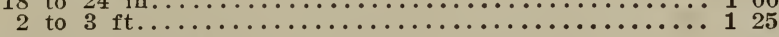

" opulifolia, White

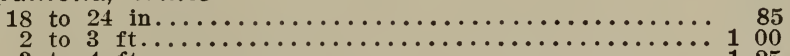

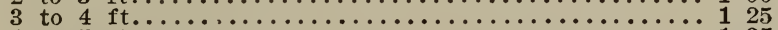

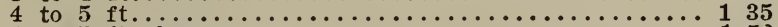

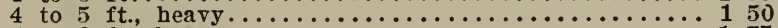

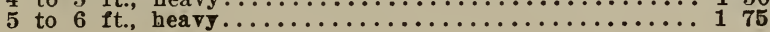

750

900

1000

$\begin{array}{rr}7 & 00 \\ 8 & 00 \\ 10 & 00 \\ 11 & 00 \\ 13 & 00 \\ 15 & 00\end{array}$ 
" opulifolia Aurea, Golden Leaf

Per 10 Per 100 Per 1000

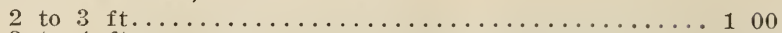

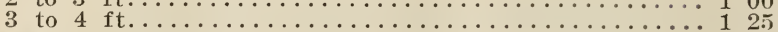

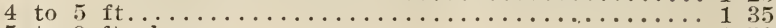

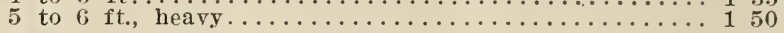

\section{prunifolia, Bridal Wreath}

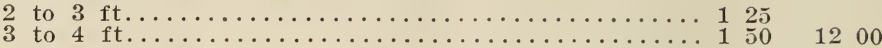

"Reevesii, Single

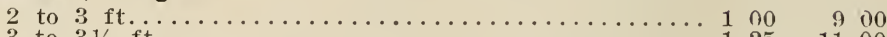

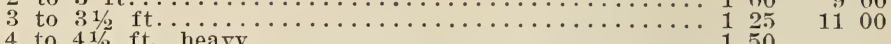

"Reevesii, Double

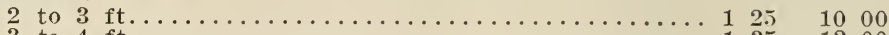

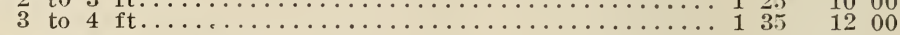

" salicifolia, White

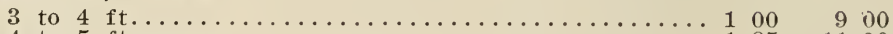

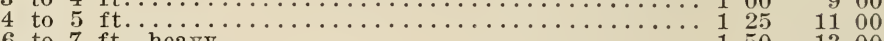

" sorbifolia

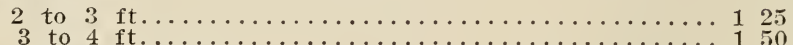

"Thunbergii, White

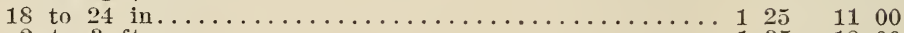

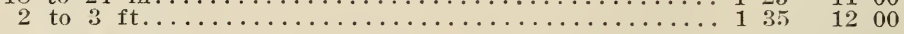

"tomentosa, Pink

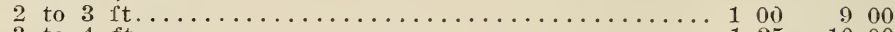

"vaccinifolia

1000

accinifolia

“Van Houttei

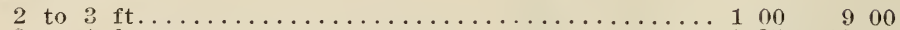

8000

Staphylea bumalda

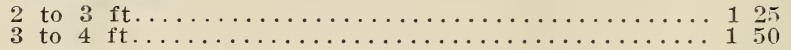

" trifoliata.

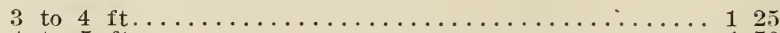

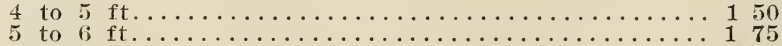

Stephanandra flexuosa

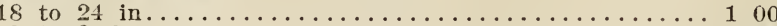

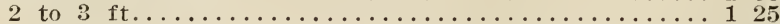

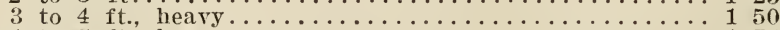

4 to 5 ft., heary......................

\section{Styrax Japonica.}

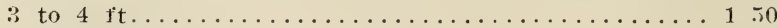

Symphoricarpus racemosus, White Snowberry

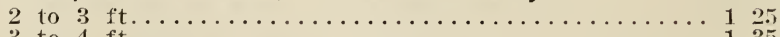

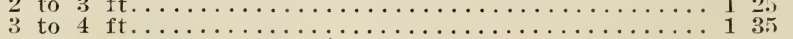

" racemosus mollis, Large White-berried

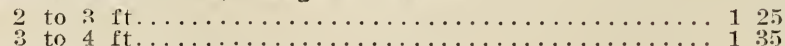

"vu!garis, Indian Currant

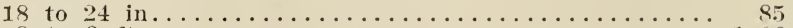

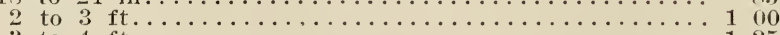

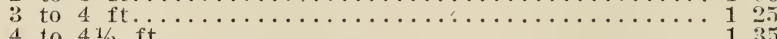

$\begin{array}{lll}7 & 00 \\ 8 & 0 & 0\end{array}$

$\begin{array}{rr}8 & 00 \\ 10 & 00\end{array}$

9000

Syringa Japonica, Japanese Tree Lilac

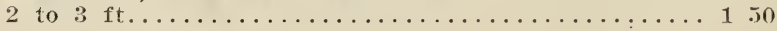

"Emodi, White, late

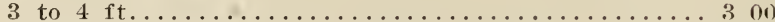

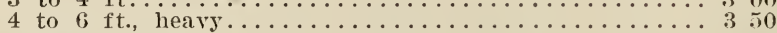

" Josikaea, Violet

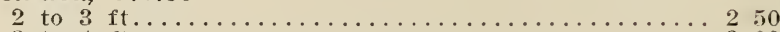

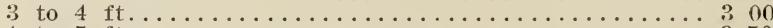

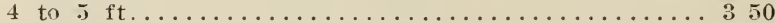


“Pekinensis

Per 10 Per 100 Per 1000

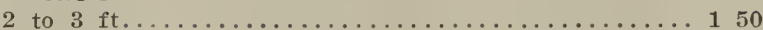

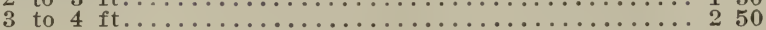

"Persica

4 to $5 \mathrm{ft}$, heary...................... 200 1800

"Persica alba

2 to 3 ft........................... 1501500

"Rothomagensis (Chinensis), Red Persian

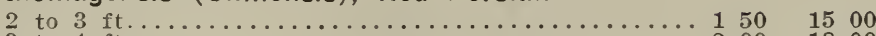

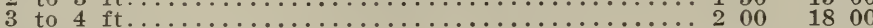

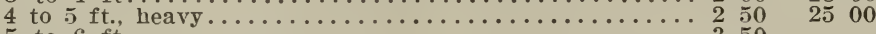

" vulgaris, Common Purple Lilac

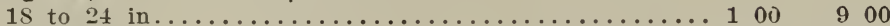

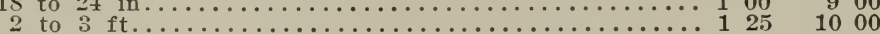

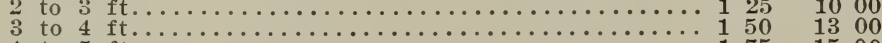

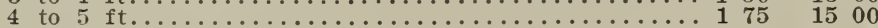

"vulgaris alba, Common White Lilac

18 to 24 in................................ 00 900

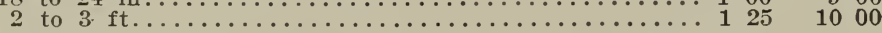

\section{Choice Named Lilacs, Single and Double}

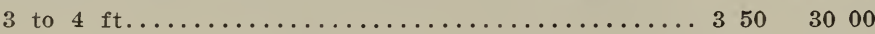

\section{LIST OF VARIETIES OF NAMED LILACS.}

ALPHONSE LAVALLE, double. Large panicles, blue, shaded violet. BELLE DE NANCY, double. Large satiny rose, white centre. Fine. CHARLES $\mathrm{X}$, single. Large, reddish purple.

FRAU DAMMANN, single. White, panicles very large.

LUDWIG SPAETH, single. Purplish red. Pan:cles large. Very fine.

MARIE LEGRAYE, single. White. Panicles large. Dwarf.

MME. ABEL CHATNEY, double. White. Large panicles.

PRESIDENT GREVY, double. Blue. Flowers very large.

SENATOR VOLLAND, double. Rosy Red. Very fine.

\section{Tamarix Africana}

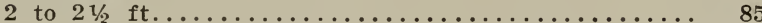

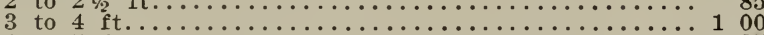

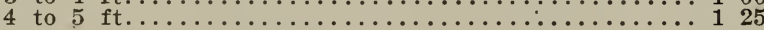

“ Gallica

18 to 24 in............................... 85

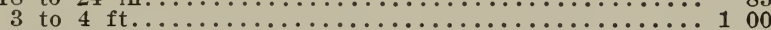

"Indica

18 to 24 in........................... $85 \quad 750$

“ Odessana

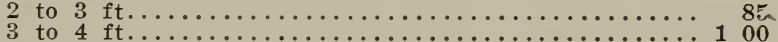

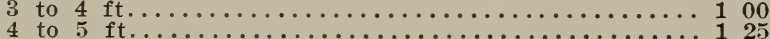

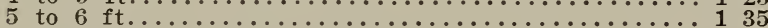

$\begin{array}{rr}9 & 00 \\ 10 & 00\end{array}$ 1200

Viburnum cassinoides

18 to 24 in.......................... $175 \quad 1500$

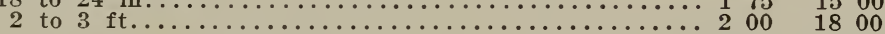

"dentatum, Arrow Wood

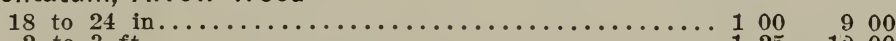

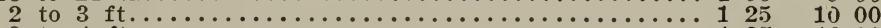

"dentatum Molle

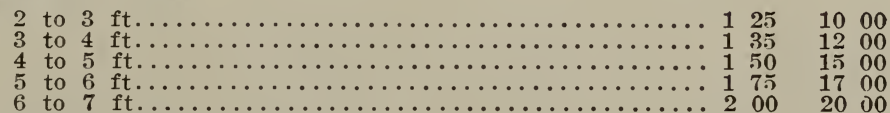

"Japonicum (Sieboldi)

2 to $3 \mathrm{ft}$, bushy...................... $250 \quad 2500$

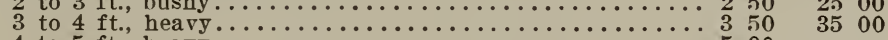

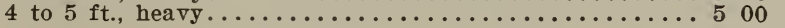


“ Lantana, Hobble Bush

Per 10 Per 100 Per 1000

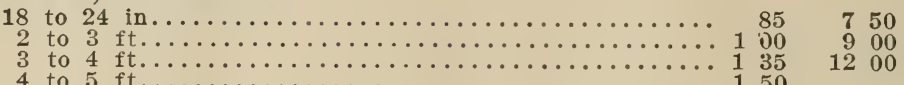

“ lentago

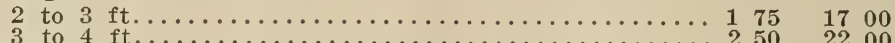

"opulus sterilis, Common Snowball

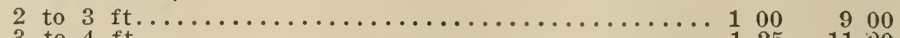

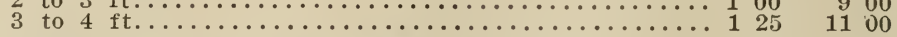

" oxycoccos (opulus), High Bush Cranberry

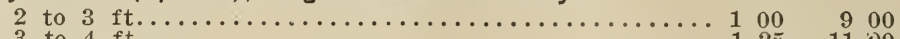

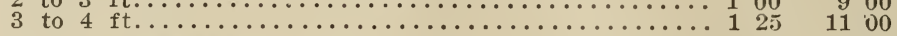

"plicatum, Japanese Snowball (true)

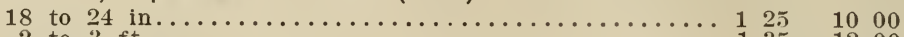

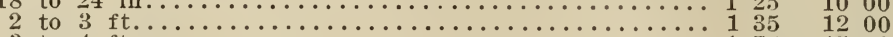

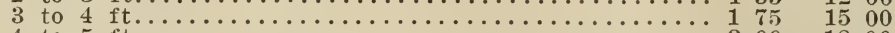

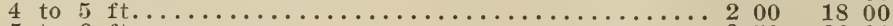

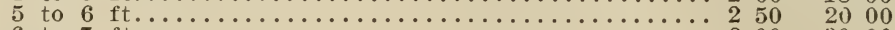

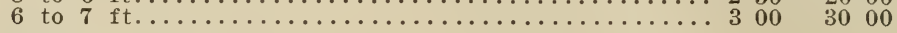

" plicatum rotundifolium

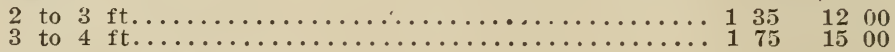

"prunifolium, Black Haw

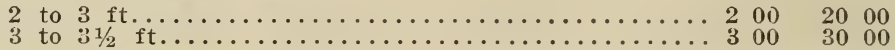

"Sieboldi (See Japonicum)

"tomentosum

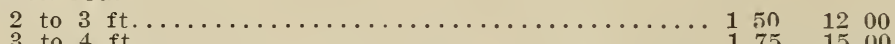

Vitex Agnus Castus, Blue

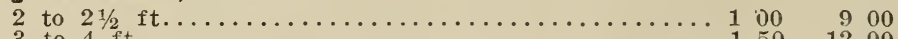

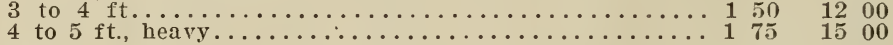

"incisa

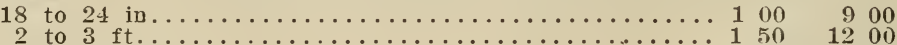

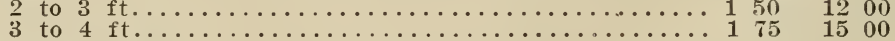

Weigela amabilis

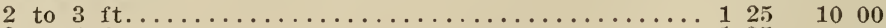

" arborea versicolor

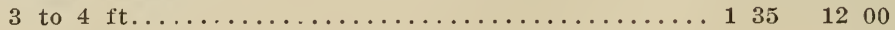

" candida, White

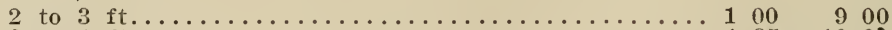

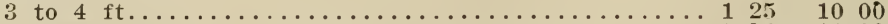

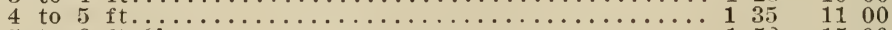

5 to 6 ft. heavy.....................

“Eva Rathke, Crimson

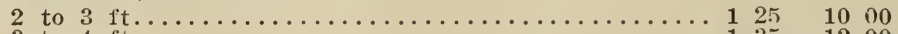

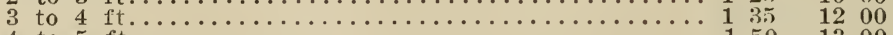

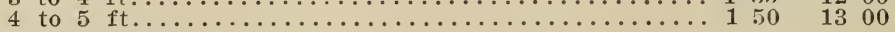

“ floribunda, Crimson

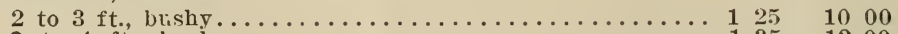

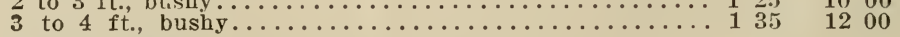

“ nana variegata

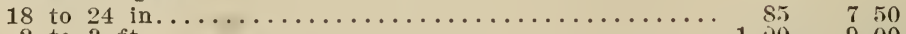

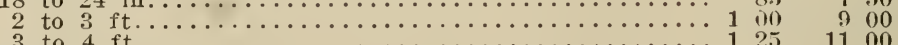

"rosea, Pink

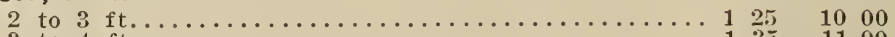

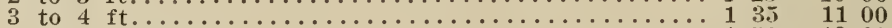

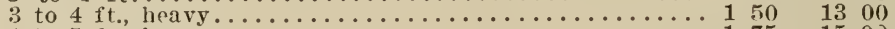

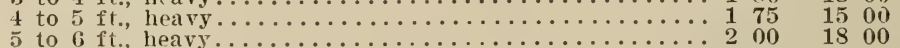




\section{Evergreen Shrubs}

Abelia rupestris

Per 10 Per 100 Per 1000

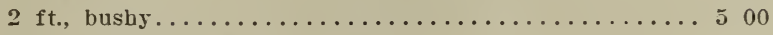

Azalea amoena, Evergreen Azalea

18 to 24 in., 5 in. pots....................... 400

“ amoena Hinondigiro. Bright carmine

12 to 15 in., bushy, 6 in. pots................. 00

Berberis ilicifolia, Holly-leaved Barberry

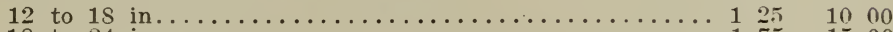

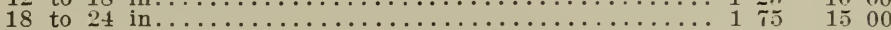

Buxus Arborescens, Bush form

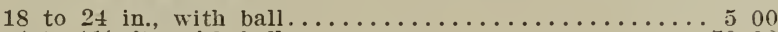

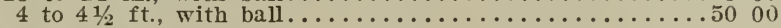

" sempervirens

9 to 10 in., bushy, with ball ................... 175

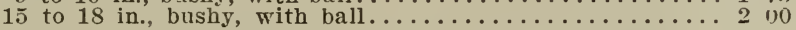

The 9 to 10 and 15 to 18 inch box bush are especially grown for edging. They are good bushy plants and each plant carries a small ball of earth. They are just the stock to use where an immediate effect is required.

Calluna vulgaris, Heath

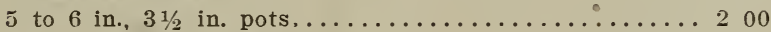

$\begin{array}{lllll}15 & 00 & 150 & 00\end{array}$

$1800 \quad 18000$

5 to 6 in., $3 \frac{1 / 2}{2}$ in. pots.
Crataegus Pyracantha Lalandi

12 to 18 in., with ball....................... 50

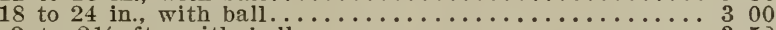

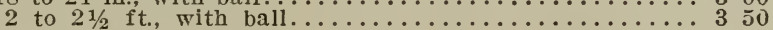

Daphne cneorum

8 to 10 in., with ball................... 400

Erica stricta

5 to 6 in., $3 \frac{1 / 2}{}$ in. pots........................ 2001500

" vagans capitata

5 to 6 in., $31 / 2$ in. pots............................... 001500

These Ericas or "Heath," as they are com-

monly called, are very nice for edging ever-

green beds. They are quite hardy.

\section{Kalmia latifolia}

18 to 24 in., with ball...................6 00

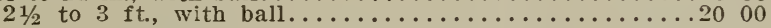

6000

Bushy clumps, transplanted last spring.

Leucothaea Catesbaei

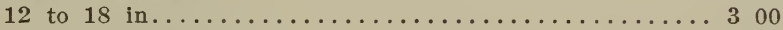

Mahonia aquifolia

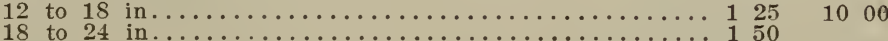

* Japonica

18 to 24 in., bushy.......................... 350

Rhododendron punctatum, Early flowering Rhododendron

15 to 18 in., bushy, with ball.............. 50

These are good bushy clumps, acclimated and transplanted last spring. 


\section{Evergreens}

We feel that we cannot say too much of our evergreens. We give particular care and attention to their culture, transplanting them as frequently as may be necessary in order to secure an abundance of fibrous roots. To this end they are transplanted every two years, and frequently sheared so that thny have a good compact and shapely appearance.

All evergreens are dug with ball of earth and the ball sewed in burlap, the cost of which is included in the prices shown in the catalogue.

Per 10 Per 100 Per 1000

\section{Abies (See also Picea)}

\section{Abies Canadensis (See Tsuga Canadensis)}

"Douglassi, Douglass Fir

18 to 24 in., with ball................... 750

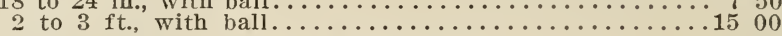

" Nordmanniana, Nordman's Silver Fir

Biota (See Thuya)

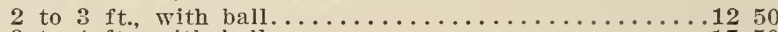

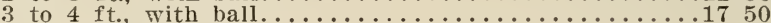

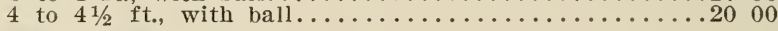

Juniperus communis, Upright form

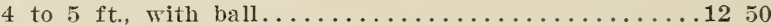

" Hibernica, Irish Juniper

2 to 3 ft., with ball................... 300

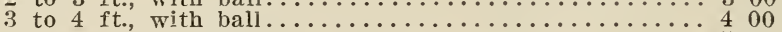

"Sabina

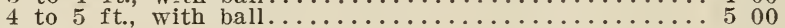

$\begin{array}{rrr}25 & 00 \\ 35 & 00\end{array}$

3500

18 to 24 in., with ball.................. 4 . $00 \quad 3500$

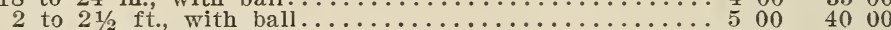

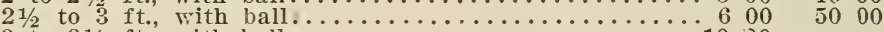

"Schottei

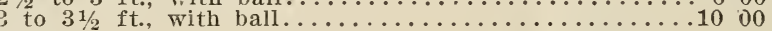

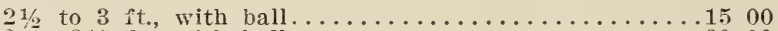

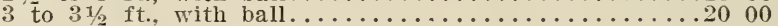

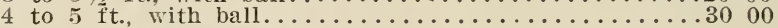

“ sinensis argentea variegata

18 to 24 in., with ball................... 500

“

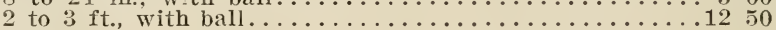

stricta

18 to 24 in., with ball.................. 500

" suecica compacta

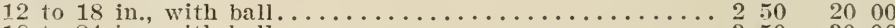

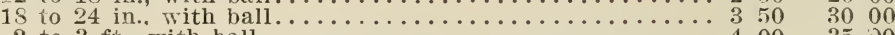

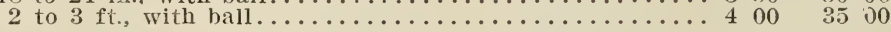

\section{"Tamaricifolia}

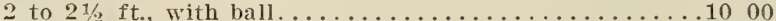

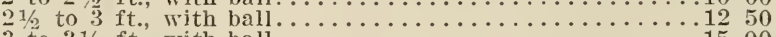

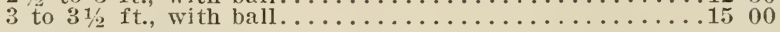

"Virginiana glauca, Blue Red Cedar

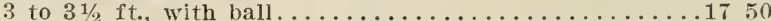

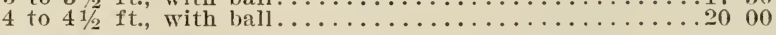

Picea (see also abies)

" excelsa inverta

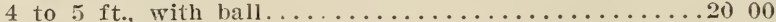

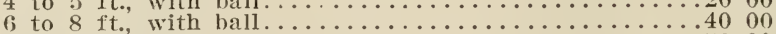

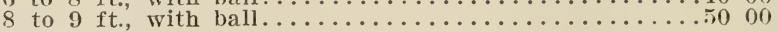

" Orientalis, Oriental Spruce

$2 \frac{1 / 2}{2}$ to $3 \mathrm{ft}$, with ball........................ 50

“ pungens, Koster's Blue Spruce, selected·Blue

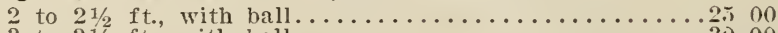

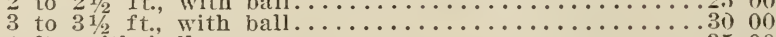

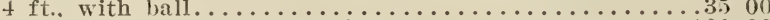

$41 \%$ to 5 ft.. with ball, specimens................. 0000 


\section{Retinisporas}

We have probably the largest and finest stock of Retinissporas, in a!l sizes, in the country. Plants from 1 foot to 11 feet. All sheared in good compact form and many times transplanted. Every plant is dug with a large ball of earth and the ball sewed in burlap. Even our smallest plants can be termed "a specimen plant." Considering the quality of our stock, the prices quoted are more than reasonable.

\section{Retinispora eriocoides}

Per 10 Per 100 Per 1000

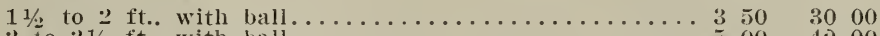

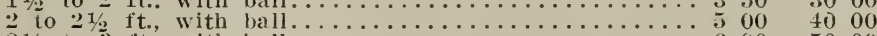

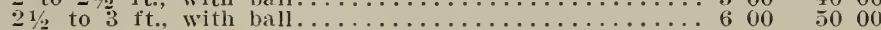

" filifera

2 to $3 \mathrm{ft}$, with ball................. 6 50

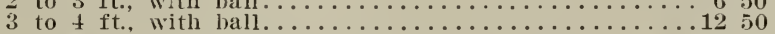

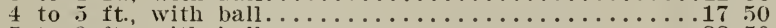

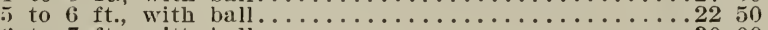

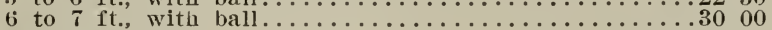

10000

" obtusa compacta

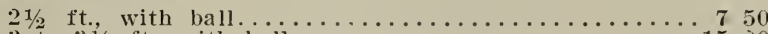

3 to $: 31 / 2$ ft., with ball................................

“ pisifera

$31 / 2$ to 4 ft., with ball...........................

3 to $4 \mathrm{ft}$, with ball..................

" pisifera aurea, Golden

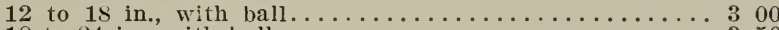

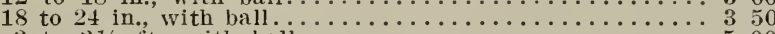

2 to $21 / 2$ ft., with ball.........................

3 to 4 ft., with ball................... 50

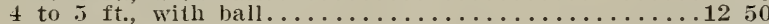

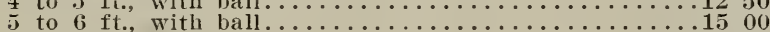

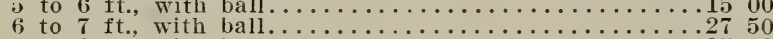

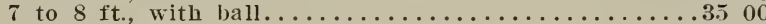

8 to $9 \mathrm{ft}$, with ball.........................

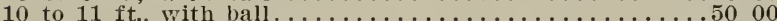

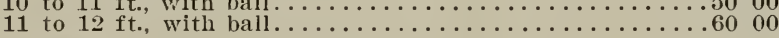

\section{" plumosa}

12 to 18 in., with ball..................... 300

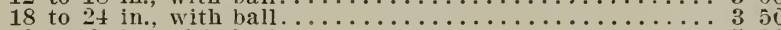

2 to 3 ft., with ball................... 500

3 to 4 ft., with ball................... 750

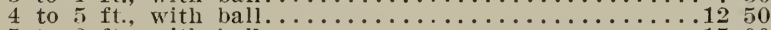

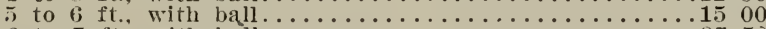

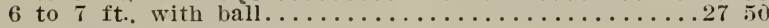

" plumosa aurea, Golden

18 to 24 in., with ball. .................. 350

2 to $3 \mathrm{ft}$, with ball................... 500

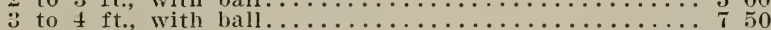

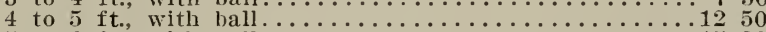

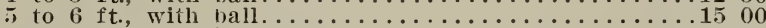

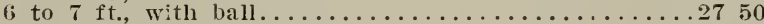

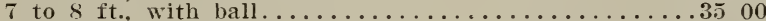

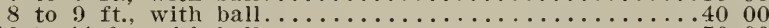

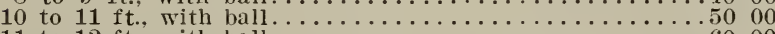

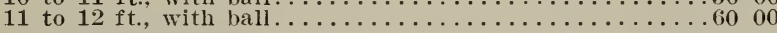

2500

3000

4000

2500

3000

4000

6000

" squarrosa Veitchii

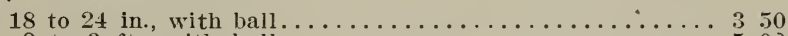

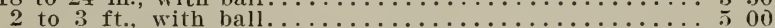

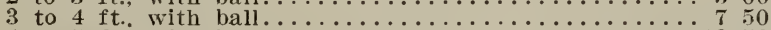

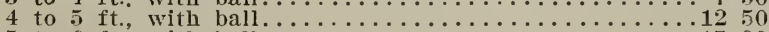

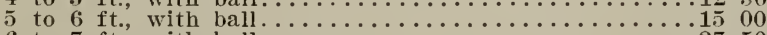

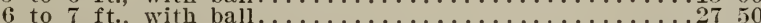

7 to $8 \mathrm{ft.}$, with ball...................... 00

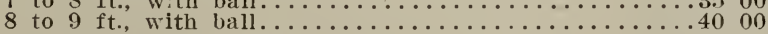

" squarrosa Veitchii aurea

4 to $4 \frac{1 / 2}{f t}$, with ball.................. 1750

Taxus cuspidata brevifolia

12 to 15 in., with ball.................. 500

3000

$40 \quad 00$

600 ) 
" Hibernica, Irish Yew

12 to 18 in., with ball.................... 400

"Hibernica aurea, Golden Irish Yew

$3 \frac{1 / 2}{2}$ to $4 \mathrm{ft}$, with ball................... 00

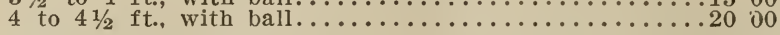

Thuya Hoveyii, Hovey's Golden Arbor Vitae

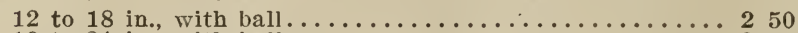

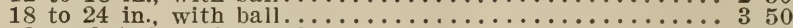

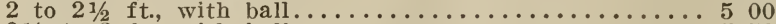

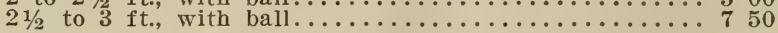

" occidentalis, American Arbor Vitae

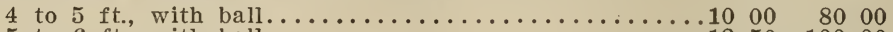

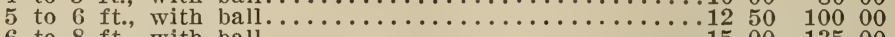

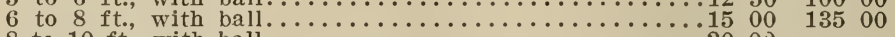

" occidentalis Geo. Peabody, Golden Arbor Vitae

9 to $10 \mathrm{ft}$, with ball......................... 00

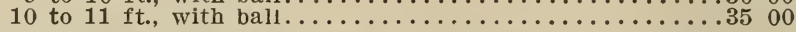

" occidentalis globosa, American Globe Arbor Vitae

10 to 12 in., with ball................... 50

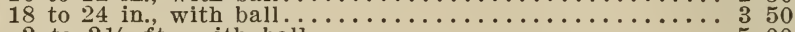

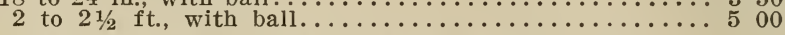

" occidentalis pyramidalis, Pyramidal Arbor Vitae

18 to 24 in., with ball.................... 50

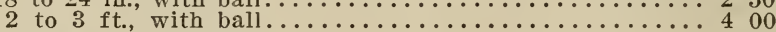

3 to 4 ft., with ball.................... 600

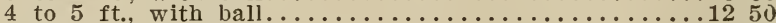

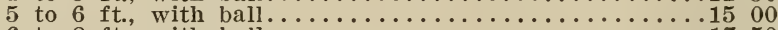

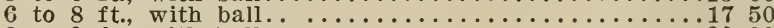

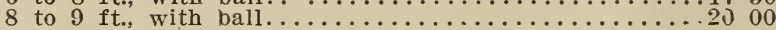

" occidentalis plicata, Fern-leaved

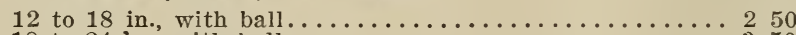

18 to 24 in., with ball................... 50

2 to $21 / 2$ ft., with ball.................... 500

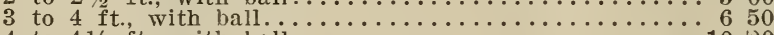

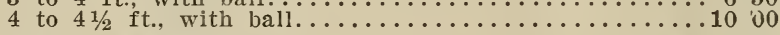

" occidentalis spiralis

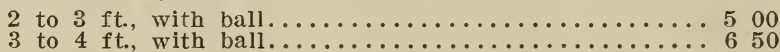

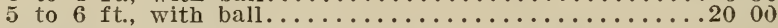

" occidentalis Vervaeneana, Golden Variegated

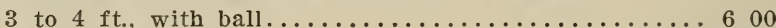

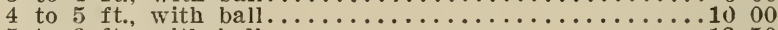

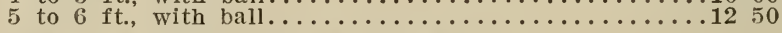

" occidentalis Victoria, Silver Tipped

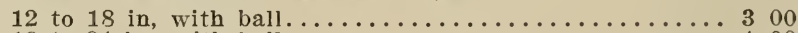

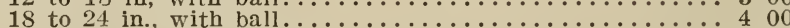

2 to $2 \frac{1}{2}$ ft., with ball................... 500

" pumila, German Globe

18 to 24 in., with ball........... 350

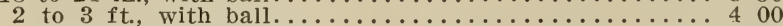

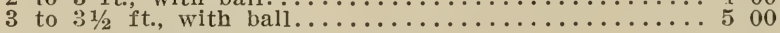

* Siberica, Siberian Arbor Vitae

12 to 18 in., with ball................... 50

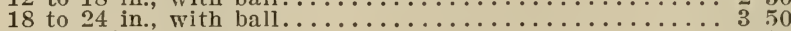

2 to $21 / 2 \mathrm{ft}$., with ball.................... 50

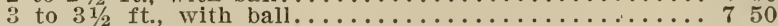

" (Biota) Orientalis, Chinese Arbor Vitae

2 to $3 \mathrm{ft}$, with ball................... 500

3 to 4 ft., with ball........................

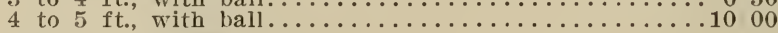

Tsuga canadensis, Hemlock

18 to 24 in., with ball.................... 350

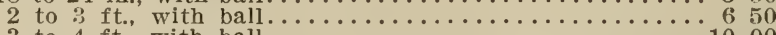

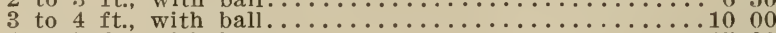

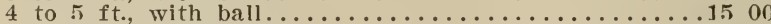

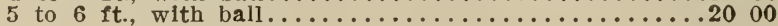




\section{Vines and Climbers}

Our vines are all transplanted plants. We handle them just as we do shrubs, grow them for one year, then transplant them into regular nursery rows, give them plenty of room so that we may get a good bushy well-rooted plant.

Particular attention should be given to the pot grown vines which we offer, they are good bushy plants, grown one summer in five-inch pots or larger as may be required. Many planters prefer pot grown plants for setting out in the Fall, as they are less liable to "heave out" with the frost than field grown stock, and besides this these plants will produce a stronger and more vigorous growth next Summer than the field grown stock.

When shipping these plants we knock them out of the clay pots in which they are grown and put them in paper pots. In this way the plants are delivered to customer with the ball unbroken.

Actinidia arguta

Per 10 Per 100 Per 1000

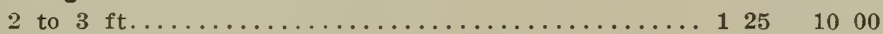

Akebia quinata

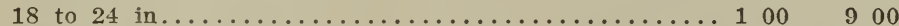

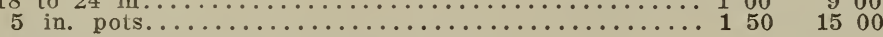

Ampelopsis Veitchii, Japan or Boston Ivy

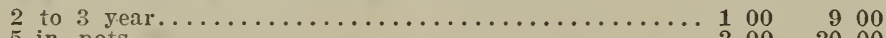

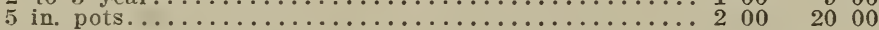

"Engelmanni

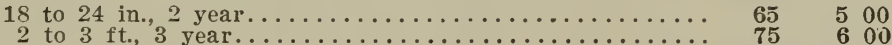

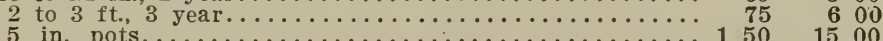

"Virginica (quinquefolia), Virginia Creeper

18 to 24 in., 2 year......................... $85 \quad 700$

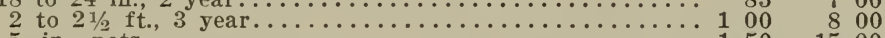

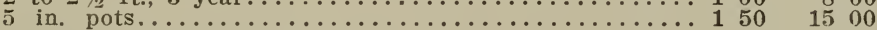

Aristolochia Sipho, Dutchman's Pipe

18 to 24 in., 2 year........................ 5 .

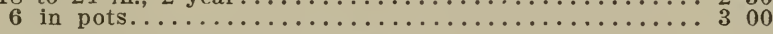

Bignonia radicans, Trumpet Creeper

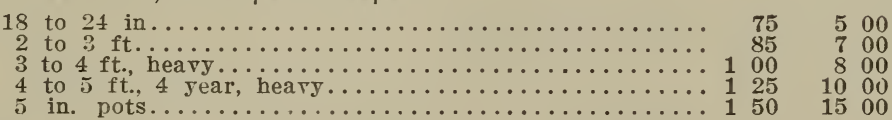

" radicans aurea, yellow

5 in. pots............................. 50

" radicans grandiflora

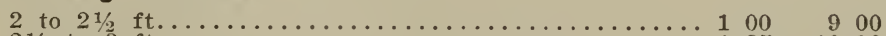

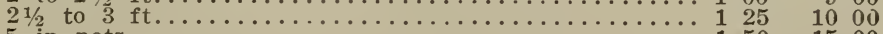

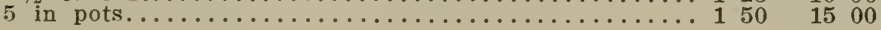

Celastris orbiculatus

5 in. pots............................ 00

2000

“punctatus

5 in. pots........................... 
" scandens, Staff Vine

P'er 10 Per 100 P'er 1000

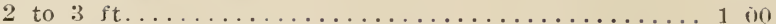

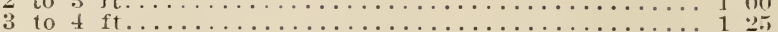

in. pots....................... 15.500

\section{Clematis paniculata}

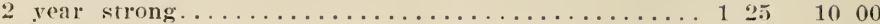

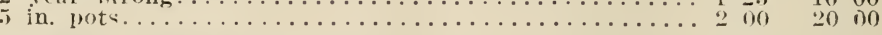

"Virginiana. Virgin's Bower

4 year ......................... 1251000 5 in. pots......................

\section{CLEMATIS HYBRIDS}

Strong :3 year, ; in. pots................ $250 \quad 2500$

\section{LIST OF VARIETIES OF CLEMATIS}

GYisY OUEEN, Dark Blue.

HENRYII, White.

JACKMANNI, Purple.

LAWSONIANA, Light Blue.

MME. ED. ANDRE, Red.

SIEBOLDII, Lavender

\section{Decumaria barbara}

Strong roots, pot giown................. 250

\section{Euonymus radicans, Green-leaved}

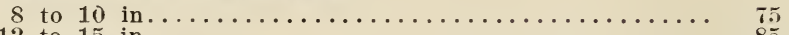

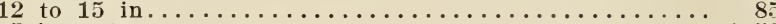

600

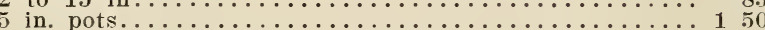

" radicans variegata

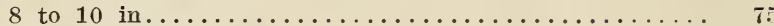

5 year, heavy clumps.................... 100

5 in pots.

Hedera Helix, English Ivy

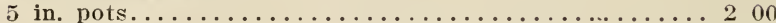

Humulus Iupulus, Hop Vine

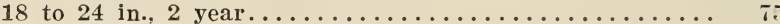

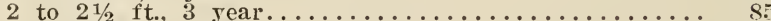

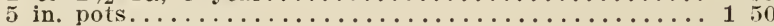

" lupulus aurea

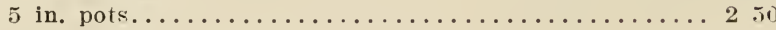

Jasminum nudiflorum, Yellow Jessamine

2 year ............................. 150

5 in. pots.............................. 50

" officinale, White Jessamine

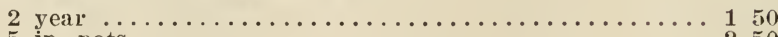

"primulinum

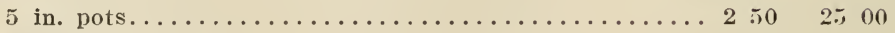

\section{Lonicera Belgica}

5 in. pots................... 1 n

\section{" brachypoda}

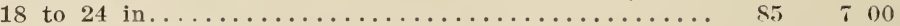

" brachypoda aurea, Golden Honeysuckle

18 to 24 in ........................ 85 700

5 in. pots............................. 150 15 00 


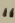

Glauca

Per 10 Per 100 Per 1000

2 to $3 \mathrm{ft}$

$125 \quad 1000$

"Halleana. Hall's Honeysuckle

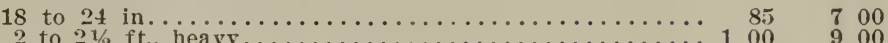

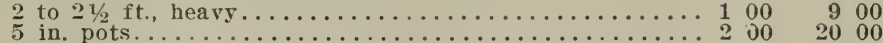

“ Japonica

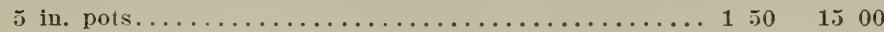

"Punicea

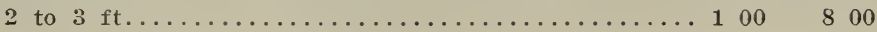

"Red Coral

2 to 3 ft....................... $100 \quad 800$

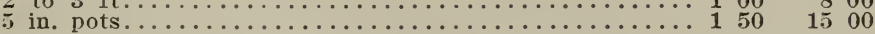

" sinensis, Chinese Honeysuckle

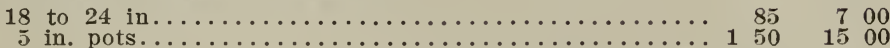

"Sullivanti

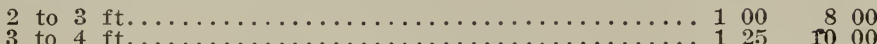

Lycium barbarum, Matrimony Vine

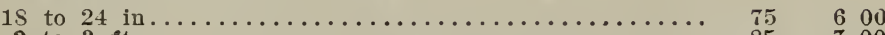

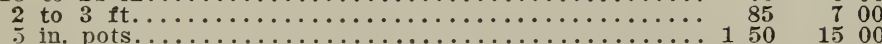

Menispurmum canadense

2 year, strong......................... 150

Periwinkle, See Vinca minor

Roses, Climbing, Creeping and Pillar (See Shrubs)

Tecoma radicans, See Bignonia radicans

Vinca minor, Periwinkle

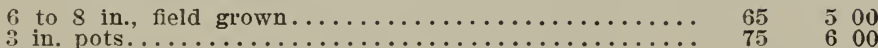

Vitis heterophylla variegata

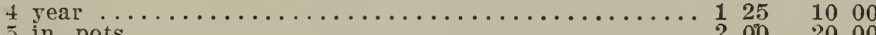

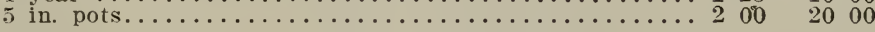

" cordifolia

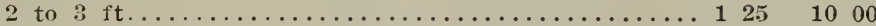

" riparia

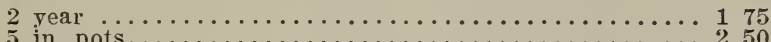

Wistaria frutescens, American Glycine ..

5 in. pots......................... 00

" sinensis, Chinese Blue Wistaria

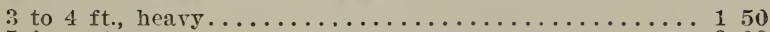

5 in. pots............................. 300

" sinensis, Tree Form

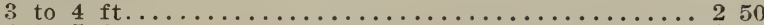

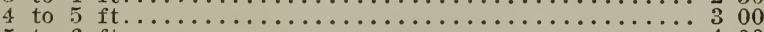

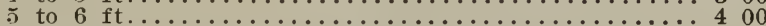

" sinensis alba, Chinese White Wistaria

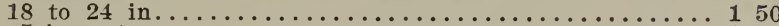

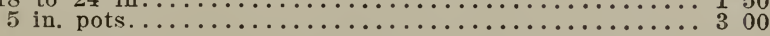




\section{HARDY HERBACEOUS PERENNIALS, ORNAMENTAL GRASSES AND FERNS}

Here is a splendid assortment of the very choicest hardy perennials for hardy gardens, borders and formal gardens.

Not small, weak, microscopic specimens, but strong, well rooted plants, capable of producing good, quick results.

"Results the first season" is our aim always, and the general satisfaction our stock is giving is conclusive proof of its superiority.

July to September, etc., indicates the period of flowering.

Achillea millefolium ros 10 Per 100

Sept. $80 \quad 600$

" Ptarmica plena "The Pearl," double white, July....... $80 \quad 600$

" tomentosa, golden yellow, July.................. $80 \quad 600$

Aegopodium podagraria variegata, variegated foliage........ $80 \quad 600$

Agrostemma coronaria, MULLIEN PINK, crimson, June..... $100 \quad 800$

Ajuga genevensis, BUGLE, blue, May................ $80 \quad 600$

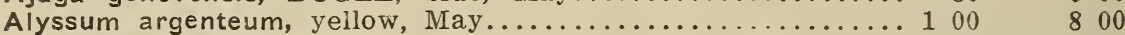

" saxatile compactum, golden yellow, May........... $80 \quad 600$

Amsonia salicifolia, blue, June..................... $100 \quad 800$

" tabernaemontana, clear blue, June ............. $100 \quad 800$

Anchusa Italica, dark blue, June to Sept............... $100 \quad 800$

Anemone Japonica alba, WINDFLOWERS, white, Sept. to Nov.. $100 \quad 800$

“Japonica rosea, rose, Sept. to Nov................... $100 \quad 800$

" Japonica "Queen Charlotte," La France pinl, Sept. to

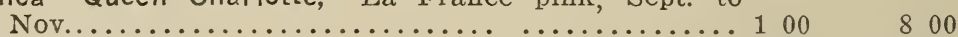

" Japonica "Whirlwind," white, Sept. to Nov............. 1008800

“Japonica rubra, red, Sept. to Nov................ $100 \quad 800$

" sylvestris, white, April and May......................... 100.800

Anthemis tinctoria, CHAMOMILE, yellow, July........... $810^{\circ} \quad 600$

Anthericum liliastrum, white, May ..................... $80 \quad 600$

Aquilegia Canadensis, COLUMBINE, scarlet, May and June.... 1000800

"caerulea, blue and white, May.................. $100 \quad 800$

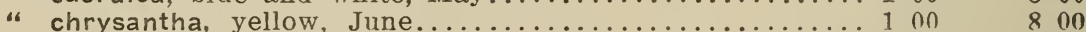

“ flabellata nana alba, large white, June .............. $100 \quad 800$

“ Skinneri, red and yellow, May................... $100 \quad 800$

“ vulgaris, blue and purple. May................... $100 \quad 800$

“vulgaris fl. pl., various, May.................. $100 \quad 800$

“ vulgaris alba, white. May....................... 1008800

Arabis albida, ROCK CRESS, white, April.................. $100 \quad 800$

Armeria maritima alba, THRIFT, white, May............ $100 \quad 800$

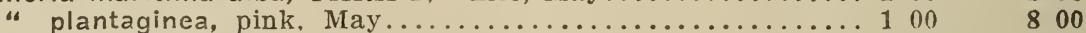

Artemisia Abrotanum, OLD MAN ............................ $80 \quad 800$

" pontica, OLD WOMAN, silver foliage ............. $80 \quad 600$

Asclepias tuberosa, BUTTERFLY PIANT, orange, July..... $100 \quad 800$

Aster alpina, bright purple, June and July............... $80 \quad 600$

“ amethystinus, large pale blue, Sept.................... $80 \quad 600$

“ Novae-Angliae, purple ................................. $80 \quad 600$

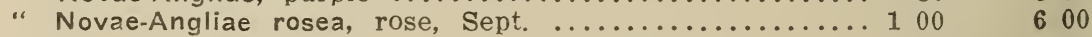

“ Novi-Belgii Robert Parker, pale blue, Sept................. $100 \quad 800$ 
Per 10

“ Novi-Belgii Top Sawyer, lavender, Sept........... 100

"Novi-Belgii White Queen, white, Sept.............. 100

" ptarmicoides, white, July and August............... 100

" Pyrenaicus, white, July and August................ 100

"Tataricus, light purple, late fall................... 80

Baptisia Australis, FALSE INDIGO, blue, June............ 100

Belamcanda Chinensis, BLACKBERRY LILY, red orange, Aug.

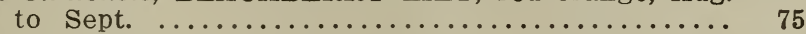

Bellis perennis, ENGLISH DAISY, various colors........... 60

Betonica orientalis, BETONY, pink, July ............... 80

" rosea, dwarf, pleasing pink, July................ 100

Bocconia cordata, PLUME POPPY, buff, June to August...... 100

Boltonia latisquama, STARWORT, pale pink, Aug. to Sept... 80

Campanula carpatica, BELLFLOWERS, blue, July to Sept..... 100

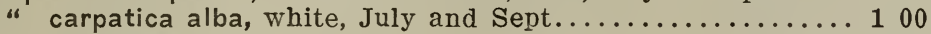

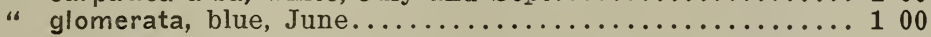

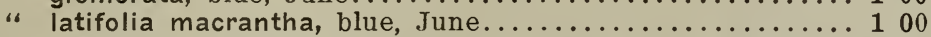

" medium, CANTERBURY BELLS, blue, Mà and June... 100

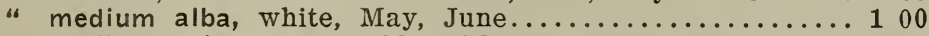

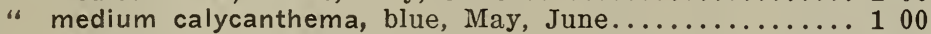

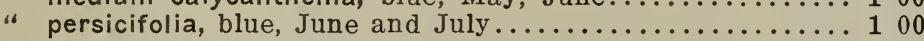

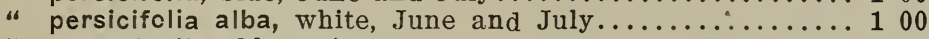

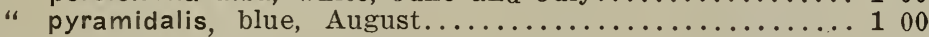

" rotundifolia, light blue, June .................... 80

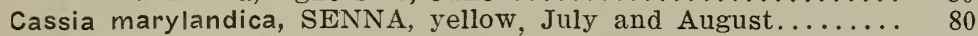

Centaurea dealbata, CORNFJ,OWERS, yellow, Aug. and Sept... 100

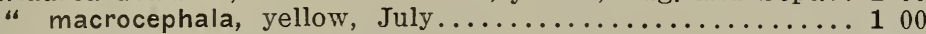

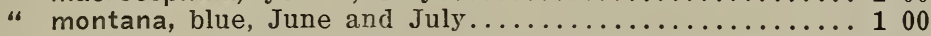

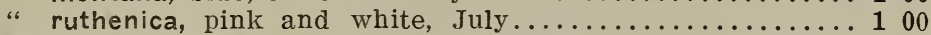

Cerastium tomentosum, SNOW IN SUMMER. MOUSE EAR,

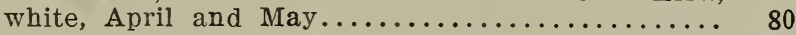

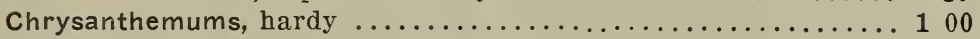

Plants from five inch pots, $\$ 1.50$ per 10.

Per 100

800

800

800

800

600

800

500

400

600

800

800

600

800

800

800

800

800

800

800

800

800

800

600

600

800

800

800

800

600

800

\section{LIST OF HARDY CHRYSANTHEMUMS}

\begin{tabular}{|c|c|c|c|}
\hline \multicolumn{3}{|c|}{ Size of Flower } & Height \\
\hline Autumn Queen & Large & Rosy pink & Medium \\
\hline Brown Pessie & Button & Beautiful Brown & Medium \\
\hline Eagle d'Or & Medium & Good, clear yellow & Tall \\
\hline Edna & Medium & Glowing Pink & Dwarf \\
\hline Flora & Small & Golden yellow & Dwarf \\
\hline Fire Ball & Medium & Yellow, tipped crimson & Medium \\
\hline Golden Mlle. Martha & Small & Clear orange yellow & Tall \\
\hline Golden Pheasant & Small & Rich Yellow & Medium \\
\hline Goldfinch & Small & Gold, shaded crimson & Tall \\
\hline Julia Lagravere & Large & Rich maroon & Tall \\
\hline Kadar & Medium & Mottled crimson, garnet & Dwarf \\
\hline Ladysmith & Medium & Rosy lake, tinged salmon & Tall \\
\hline L'Ami Conderschlerdt & Small & Sulphur white & Dwarf \\
\hline La Favorite & Small & Rosy pink, shaded white & Medium \\
\hline Little Pet & Small & Claret, perfect form & Tall \\
\hline Lovely. & Small & Bright pink & Tall \\
\hline Model & Simall & Round, full white & Tall \\
\hline Mrs. Snyder & Large & Rich yellow & Medium \\
\hline Mrs. Vincent & Large & Magenta & Tall \\
\hline Nellie Rainsford & Small & Orange salmon, tipped red & Dwarf \\
\hline President & Large & Deep, violet rose & Tall \\
\hline Prince Victor & Large & Brownish red & Tall \\
\hline Souer Melaine & Large & Fine pure white & Medium \\
\hline St. Illoria & Large & Glorious silvery pink & Tall \\
\hline Strathmeath & Large & Clear pink & Medium \\
\hline Stratagem & Large & Crimson, shaded gold & Tall \\
\hline Sunset & Medium & Single, reddish brown & Medium \\
\hline
\end{tabular}

Young plants set out in spring give the best results. If desired for setting out in the fall or for propagating purposes we can supply the above varieties in strong clumps, $\$ 1.50$ per 10 . $\$ 12.00$ per 100 .

Clematis Davidiana, BUSH CLEMATIS, blue, August........ 125

" recta, white, July ......................... 150 
Convallaria majalis, LILY OF THE VALLEY, white, spring, clumps ...........................200 1500

Coreopsis lanceolata grandiflora, TICKSEED, golden yellow,

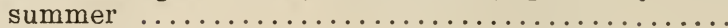

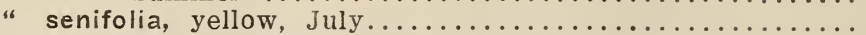

Coronilla varia, CROWN VEITCH, pink, June to Aug..........

Delphinium Chinense, CHINESE LARKSPUR, blue and white,

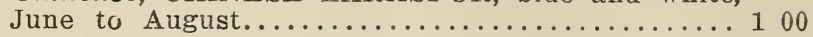

" elatum, English Hybrids, TALL LARKSPUR, shades of

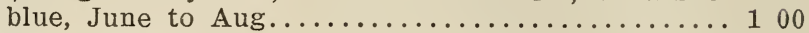

"formosum, Indigo, July....................... 100

Dianthus barbatus, SWEET WILLIAM, many colors, May and

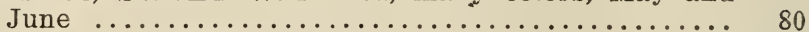

“ Lord Lyons, GARDEN PINKS, deep pink, May and June. 100

"Mirs. Sinkins, white, May and June.................. 100

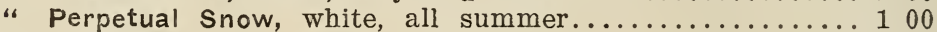

“ variabilis, white, maroon mark, May and June.......... 100

Dicentra spectabilis, BLEEDING HEART, pink, April and May. . 100

Dictamus fraxinella, GAS PLANT, red, June............ 200

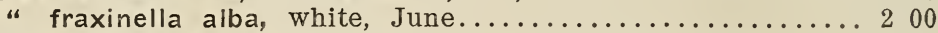

Digitalis grandiflorus, FOXGLOVE, yellow, June to July ...... 100

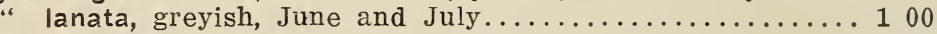

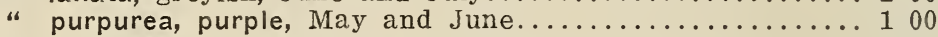

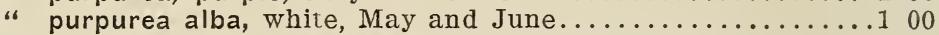

Doronicum plantagineum excelsum, LEOPARD'S BANE, yellow,

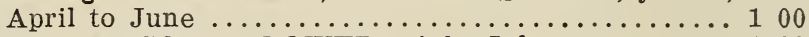

Echinacea purpurea, CONE FLOWER, pink, July.......... 100

Eryngium amethystinum, SEA HOLLY, blue, July........... 100

Eupatorium ageratoides, HARDY AGERATUM, white, Sept.... 100

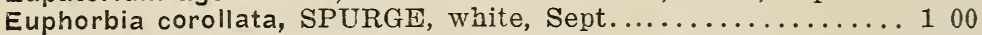

Funkia caerulea PLANTAIN LILIES, blue, July . . . . . . . . . . . 80

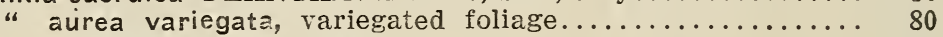

“ cordifolia, blue, August...................... 80

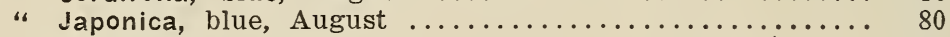

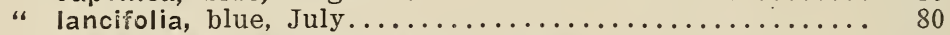

" ovata, marginata, variegated ioliage .............. 80

" subcordata grandiflora, white, August............... 100

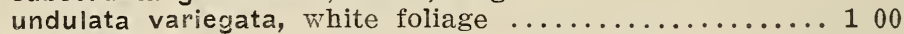

Gaillardia grandiflora, BLANKET FLOWER, yellow and crim-

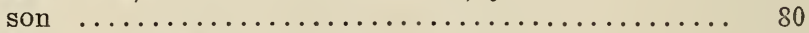

"grandiflora compacta, yellow and crimson, July to Sept.. 80

Geum coccineum, scarlet, June and July................ 100

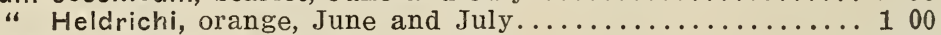

Gypsophila cerastioides, white, June................. 100

" paniculata, BABY'S BREATH, white, July and Aug..... 100

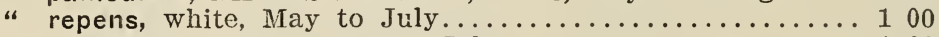

"Stevensii, white, June and July.................. 100

Helenium autumnale superbum, SNEEZEWORT, clear yellow,

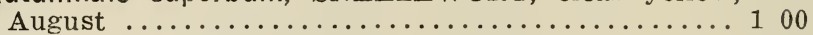

“grandicephalum striatum, yellow striped with brown, Aug. 100

"Hoopesii, orange, May..................... 100

Helianthus decapetalus multiflorus Soliel d'or, double yellow,

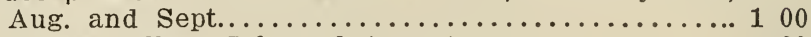

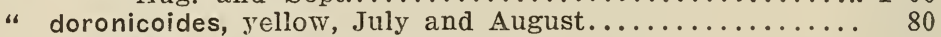

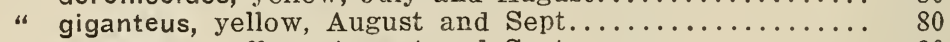

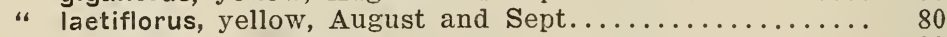

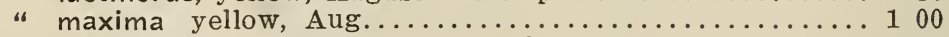

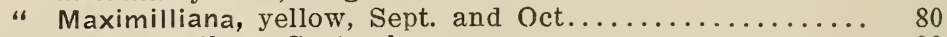

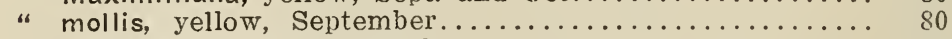

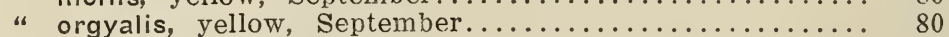

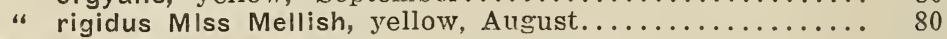

Heliopsis Pitcheriana, orange. June to August............. 80

Hemerocallis Dumortierierii, DAY LILIES, orange, June...... 100

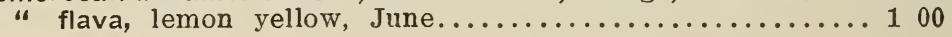

800

800

800

800

1500

1500

800

800

800

800

800

800

800

800

800

500

600

$\begin{array}{ll}6 & 00\end{array}$

600

600

600

800

800

600

600

800

800

800

800

800

800

800

800

800

800

600

600
6

600

S 00

600

600

$\begin{array}{ll}6 & 00\end{array}$

$\begin{array}{ll}6 & 00\end{array}$

600

800

800 


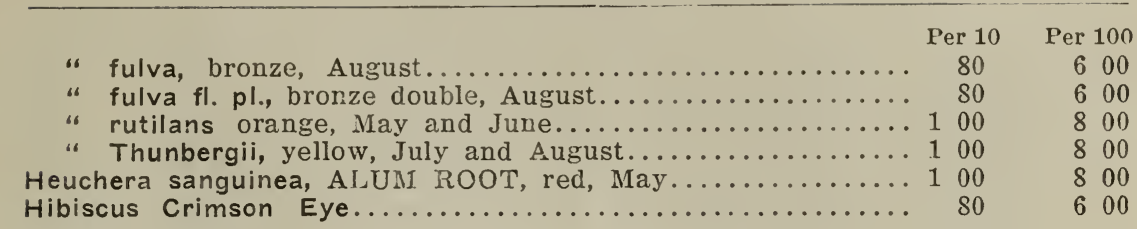

\title{
Meehans' Mallow Marvels
}

The most remarkable creation of the day. Hardy in Canada. Thrives as well there as it does in the South. A plant for all sections of the country. Individual blooms are as large as ten inches across. Plants labeled to color as they flower. Every plant sold true to color. See colored plate, last page of cover.

\section{Prices: \\ CRIMSON MARVEL \\ RED MARVEL PINK MARVEL}

$\begin{array}{lll}\text { Strong 2 year roots, } & \text { Per } 10 & \text { Per } 100 \\ \text { Strong 3 year roots, } & \$ 2.50 & \$ 25.00 \\ & \$ 4.00 & \$ 40.00\end{array}$

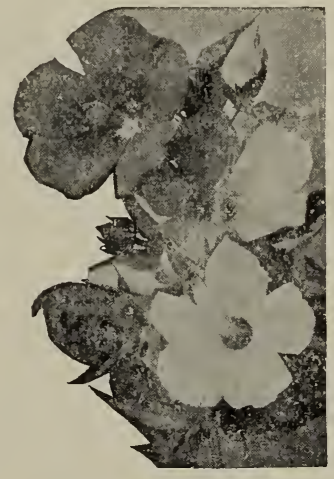

Per 10

Per 100

Hollyhock, double, separate colors, June to August......... 100

Hyacinthus candicans, SUMMER HYACINTH, white, August... 25

Iberis sempervirens, CANDYTUFT, white, April........... 80

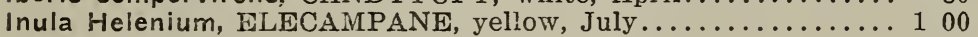

Iris Cengialti, deep lavender, May and June................ 80

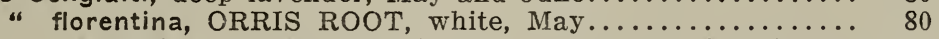

" Germanica, GERMAN IRIS, best assortment of varieties. 80

800

200

600

800

600

600

600

\section{LIST OF IRIS GERMANICA.}

\begin{abstract}
ALEXANDER YON HUMBOLDT, Light blue self.
CANARY BIRD, Canary yellow self.

COELESTINE, Pretty lavender self.

JOSEPHINE, The best dark purple.

LADY FRANCES, Delicate larender.

LADY STUMPP, Standards lavender, falls blue.

MADAME CHEREAU, White, penciled blue on edges.

MARIE, Standards lavender, falls darker.

PENEIOPE, Standards blue, falls darker.

PURPUREA, Rich purple.

ROSAMOND, Standards lavender buff, falls purple.

SILVER KING. Silvery white self.
\end{abstract}

Iris laevigata (Kaempferi), JAPANESE IRIS, in fine assorted

named kinds, July ..................... 150

1000

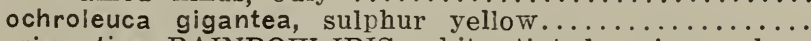

prismatica, RAINBOW IRIS, white, tinted various colors,

June ............................ 100

pseud-acorus, yellow, May ......................... 80

pumila, DWARF IRIS, Various, April................... 80

" Siberica orientale, SIBERIAN IRIS, indigo blue, June.... 100

“ spectabilis, blue, June......................... 80

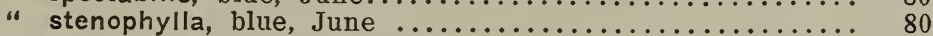

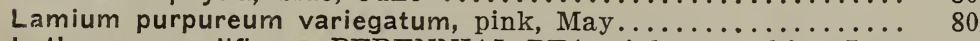

Lathyrus grandiflorus, PERENNIAL PEA, pink and white, June to August 
Lavandula spica, SWEET LAVENDER, July and August...... 100

Liatris pycnostachia, BLAZING STAR, purple, August and Sept. spicata, purple, Aug. and Sept................... so

Lilium candidum, ANNUNCIATION LILY, white, June...... 100 auratum, Gold-banded lily of Japan, white spotted, July.. 125

“ Batemanii, reddish orange, July and Aug............ 125 elegans, yellow, July and August ........................... 125

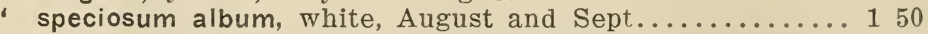

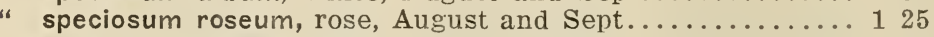

" superbum, orange red, July and August.............. 80 tigrinum splendens, TIGER LILY, orange spotted, Aug.. 80

Linum perenne, FLAX, blue, June................. S0

Lobelia cardinalis, CARDINAL FLOWER, scarlet, Aug. and Sept. 100

Lotus corniculatus TREFOIL, yellow, June to Oct......... 80

Lychnis chalcedonica, SCARLET LIGHTENING, scarlet, July . . 80

"Flos-cuculi, RAGGED ROBIN, red, May.............. 80

“ viscaria splendens, CATCH FLY, red, July.............. 80

Lysimachia clethroides, LOOSESTRIFE, white, July to Sept... 80

Lythrum salicaria, purple, June and July............... 80

" Japonicum elatum, purplish red, June and July.......... 80

" roseum superbum, purple, June and July............ 100

Monarda didyma splendens, BERGAMOT, scarlet, Aug. to Sept. $\quad 80$

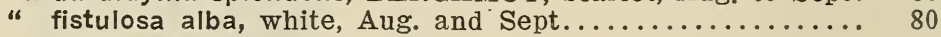

" fistulosa purpurea, purple, Aug. and Sept............ 80

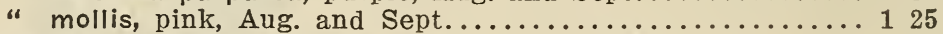

Myosotis palustris semperflorens, "FORGET-ME-NOT," blue,

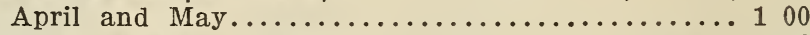

Nepeta Glechoma variegata, GROUND IVY, blue, April and May 50

Oenothera Missouriensis, EVENING PRIMROSES, yellow, July

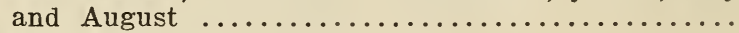

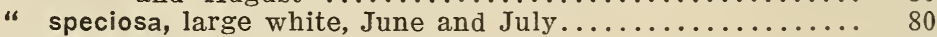

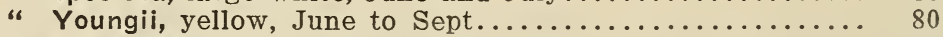

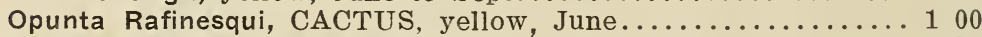

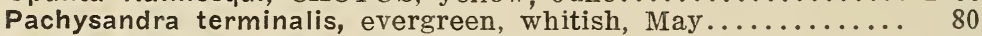

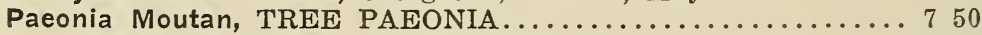

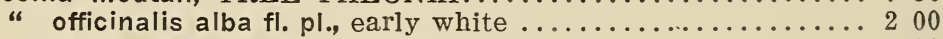

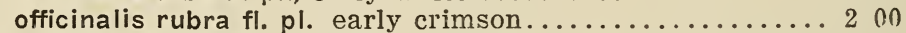

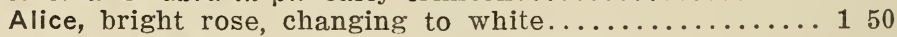

Andre Lauris, dark rose. A good standard variety...... 150

Duke of Orleans, double. Maroon Crimson.................... 300

Duke of Wellington, blush, bud centre, fragrant....... 150

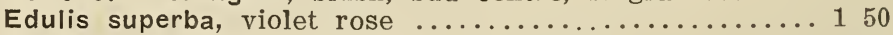

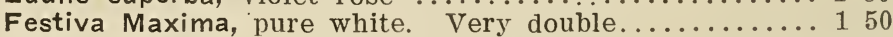

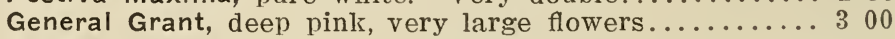

General Schofield, deep rose, full flower............. 150

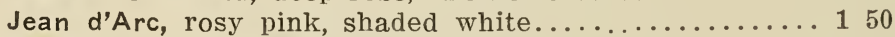

“ Leslie, crimson pink, inner petals tipped lighter....... 300

"Mrs. Fletcher, rosy pink, edged white............. 250

“Mrs. Pleas, purplish rose with straw and white centre.. 300

"Nobilissima, glowing pink, late.............. 150

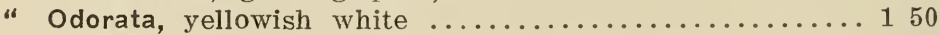

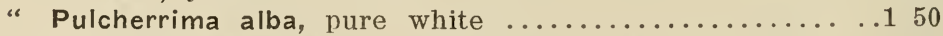

"Reine des Francais, outer petals rose color, straw centre.. 250

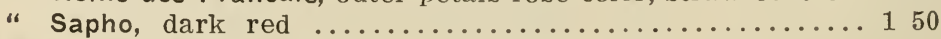

Papaver nudicaule, ICELAND POPPIES, white, yellow and

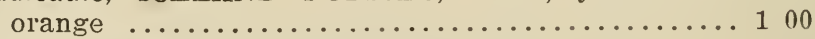

" Orientale, ORIENTAL POPPIES, crimson, June....... 100

Pentstemon barbatus hybridus, various, June............. 80

" diffusus, blue, June....................... 80

" digitalis, BEARDED TONGUE, white, July........... 80

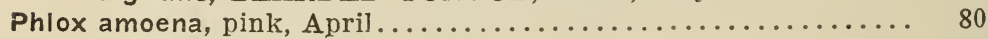

"decussata, best assortment of varieties............ 100

600

800

600

1500

1500

1200

12 00

2500

1200

12: 00

1200

2500

1200

1200

2500

2000

2500

1200

1200

1200

$20 \quad 00$

1200

800

80 n

600

600

$6 \mathrm{nn}$

600

900 


\section{LIST OF VARIETIES OF GARDEN PHLOX.}

CARRAN DE ACHE. Rosy carmine.

CHAMPS ELYSEES. Rich purplish crimson.

COQUELICOT. Fine pure scarlet, with deep carmine eye.

El'OPEE. Violet, bright fiery center.

EUGENE DANZANVILLIERS. Lilac, shading white.

INIEPENDENCE. Large pure white.

LA VAGLE. Beautiful combination of rosy pınk and lavender.

L'EVENEMENT. Salmon pink.

L.ESPERANCE. Bright liluc.

MAD. P. LANGINR. Bright red, vermillon centre.

PRINCESS LUUISE. White. small pink eye.

QUEEN. Pure white.

KICHARD WALlACE. White, crimson center.

WILLIAM ROBINSON. Tall, salmon plnk.

Phlox subulata rosea, CREEPING PHLOX, pink, April and May.

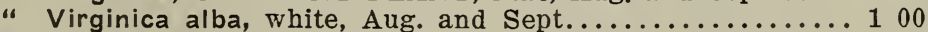

Plantago maxima, GIANT PLANTAIN, white, July........... 100

Platycodon grandiflora, JAPANESE BELLFLOWERS, blue, July

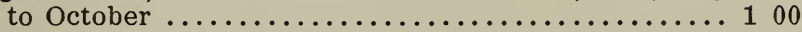

" grandiflora album, white, July to Oct............... 100

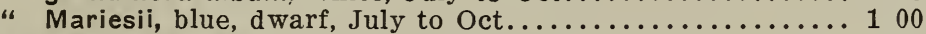

Polemonium reptans, JACOB'S LADDER, blue, May........... 80

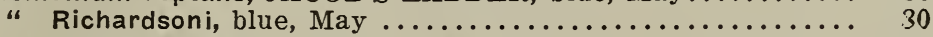

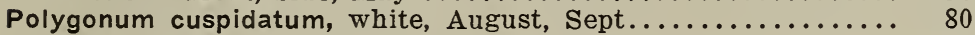

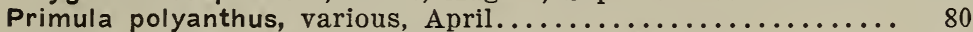

Pyrethum Balsamita, COSTMARY, fragrant foliage.......... 80

" roseum, shades of pink and red, May and June.......... 100

“ uliginosum OXEYE DAISY, white, Aug. and Sept....... 80

Ranunculus acris fl. pl., DOUBLE BUTTERCUP, golden yellow,

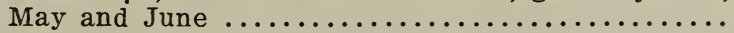

Rudbeckia fulgida, BLACK EYED SUSAN, golden yellow, July

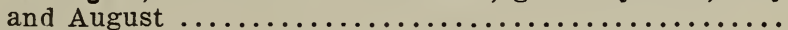

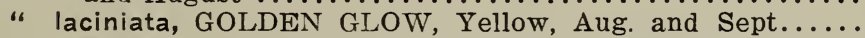

"Newmanni, BLACK EYED SUSAN, golden yellow, July

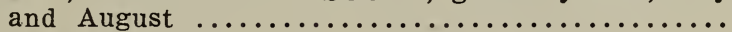

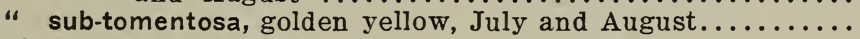

Salvia azurea grandiflora, blue, Aug.................. 100

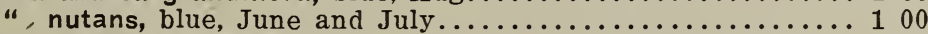

Sanguinaria canadensis BLOOD ROOT, white, April and May... 60

Santolina Incana, LAVENDER COTTON................ 80

Saponaria officinalis, BOUNCING BET, pinkish white, Aug. and

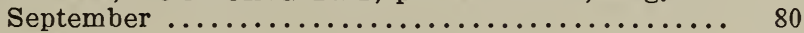

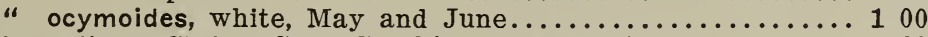

Sedum album, STONECROPS, white, Aug. and Sept.......... 80

"Hispanicum, pink, June and July................. 80

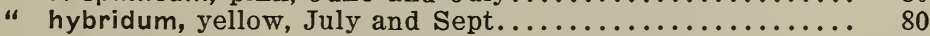

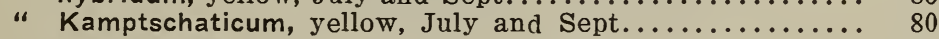

“ sexangulare, yellow, June and July.................. 80

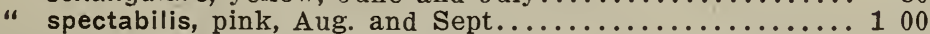

Sisyrinchium Bermudianum, STAR GRASS, blue, May......... 80

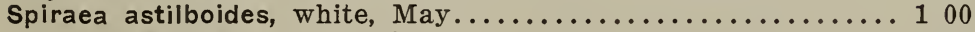

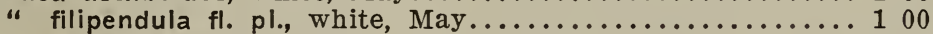

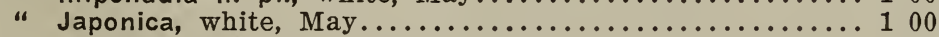

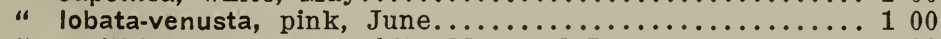

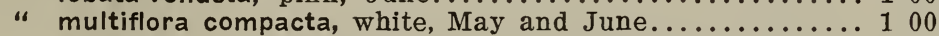

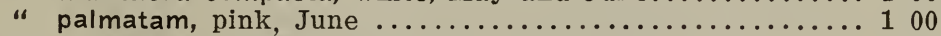

" ulmaria alba plena double white, June and July......... 80

Statice latifolia, SEA LAVENDER, August............... 100

"Gmelini, lavender, August...................... 100

Stokesia cyanea, STOKES' ASTER, blue, July to Sept....... 80

Per 100

600

600

$\begin{array}{ll}6 & 00\end{array}$

600

800

800

800

800

800

600

$\begin{array}{ll}600 \\ 6 & 00\end{array}$

600

600

600

800

600

500

600

600

600

600

800

800

400

600

600

800

600

600

600

60 ก

600

800

600

800

800

800

800

800

800

600

800

800

600 
Thalictrum adiantifolium, MEADOW RUES, handsome foliage.. 100

" aquilegifolium roseaum, pink, June ............................. 00

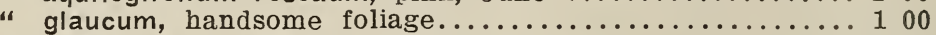

Thermopsis Caroliniana, yellow, June.................. 100

Thymus Serpyllum variegata, golden variegata............ 80

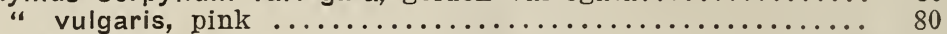

Tradescantia Virginica, SPIDER WORT, blue, May to July.... 80

"Virginica alba, white, May, July................... 80

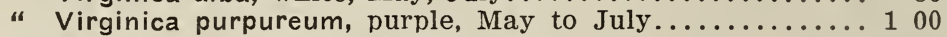

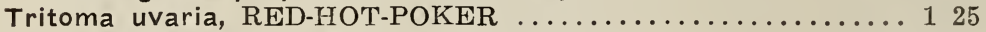

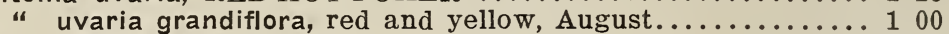

"uvaria Pfitzeri, coral, July to Oct.................. 100

Valeriana officinalis, VALERIAN, pinkish, May and June..... 100

Vernonia Jamesii, IRON WEED, purple, Aug. and Sept....... 100

Veronica candida, SPEEDWELL, silvery foliage, blue flowers,

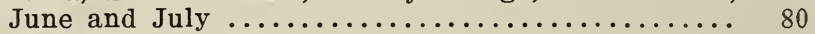

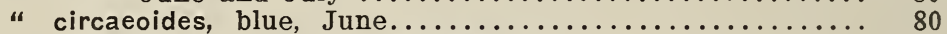

"Hendersoni (longifolia subsessilis), blue, August....... 100

"Montana, blue, June and July.................... 80

" teucrium, blue, June....................... 80

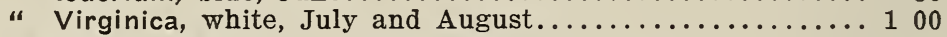

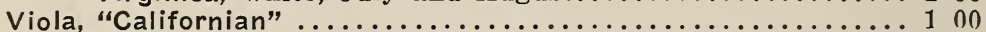

" odorata Blue, SWEET VIOLET, April and May........ 100

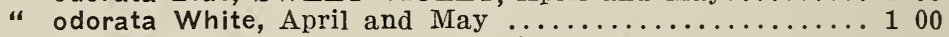

" odorata "Czar," double blue, April and May............ 10 0

Vinca minor, Periwinkle, blue, 6 to 8 in., field grown........ 65

" minor, Periwinkle, blue, 3 in. pots................ 75

Per 100

800

800

800

800

600

600

600

600

800

1000

800

800

800

800

600

600

800

600

600

800

$8 \mathrm{nn}$

800

800

800

500

600

\section{Hardy Ferns}

Aspidium acrostichoides, CHRISTMAS FERN .......... 80

“ cristatum, CRESTED SHIELD FERN .............. 80

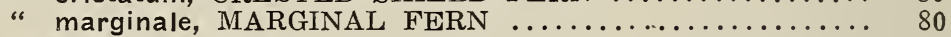

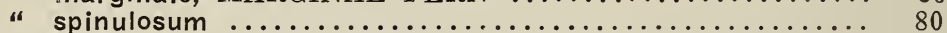

"Thelyptera, LADY FERN ................... 80

Asplenium angustifolium, NARROW-LEAVED SPLEENWORT. . 80

Onoclea sensibilis, SENSITIVE FERN …............. 80

"Struthiopteris, OSTRICH FERN .................. so

Osmunda cinnamomea, CINNAMON FERN ............. 100

"Claytoniana, FLOWERING FERN ................ 100

“ regalis, KING FERN ............................. 150

Polypodium hexagonopterum ......................... 60

vulgare, ROCK POLYPOD .................... 60

Pteris aquilina, BRAKE or BRACKEN $\ldots \ldots \ldots \ldots \ldots \ldots \ldots .60$

\section{Hardy Grasses}

Arrhenatherum bulbosum. A fine dwarf variegated grass. Useful for edging ............................. 80

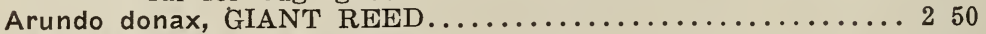

Bambusa Metake, EVERGREEN BAMBOO.............. 250

Calamagrostis stricta variegata.................... 80

Eulalia Japonica, FALSE PAMPAS GRASS............. 200

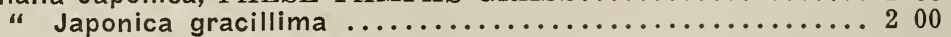

“ Japonica variegata, green and white striped............ 200

“ Japonica Zebrina, green barred with bronze, yellow....... 200

Uniola latifolia, a pretty grass with plumes, useful for vases.... 100 


\section{MEEHANS' MALLOW MARVELS}

Few new plants introduced in recent years have created as great a sensation as Meehans' Mallow Marvels.

It is the ambition of all horticulturalists to produce something that excels, something larger and better than has been grown before, but it can only be accomplished by those who have a great amount of patience, as the process is slow and more frequently ends in disappointment rather than success.

Several years ago, the late Thomas Meehan suggested the possibility of hybridizing the southern crimson-flowered mallow, which is not hardy in the North, with the white-flowered swamp mallow so common in New Jersey, and his suggestion was followed out.

Out of the first lot of seedlings produced by this cross-breeding, one seedling bore a dazzling brilliant scarlet flower. The plant was left out all winter without protection and the original plant is still growing in the nursery.

The flowers of this one plant were again hybridized as before, and again the seedlings from this plant produced a number of seedlings with various colored flowers. The best of these were saved and recrossed. This process was continued from year to year for some five or six years until a permanent strain of plants, some with crimson, some with red, and some with pink flowers, was produced. The beauty of these flowers can scarcely be described and even the colored plate fails to properly show their great beauty. Single plants set out on the lawn, in the garden, or among shrubbery beds, will create wide attention, but the greatest sensation is made when a number of plants of mixed colors are set in a bed by themselves.

Plants have been sent to various sections of the country and their hardiness fully proven. From Maine to California, from Canada to the far South. They thrive in every section.

They will grow and bloom in any soil, but do best in a rich, moist soil. They like plenty of water, but they should not be planted in a swamp where the water lies around the roots.

Flowers produced on plants which are in the most thrifty condition will measure twelve inches in diameter. They commence to bloom about the first of August and continue with a succession of flowers until frost.

The plants are of herbaceous character, dying to the ground every fall, but sprouting up again the following spring, stronger than ever. In rich soil, with plenty of moisture, a three-year-old plant will produce stems six to eight feet in height in one year. 
